\title{
ERGONOMIC AND SAFETY INSIGHTS IN MINIMAL INVASIVE SURGERY
}

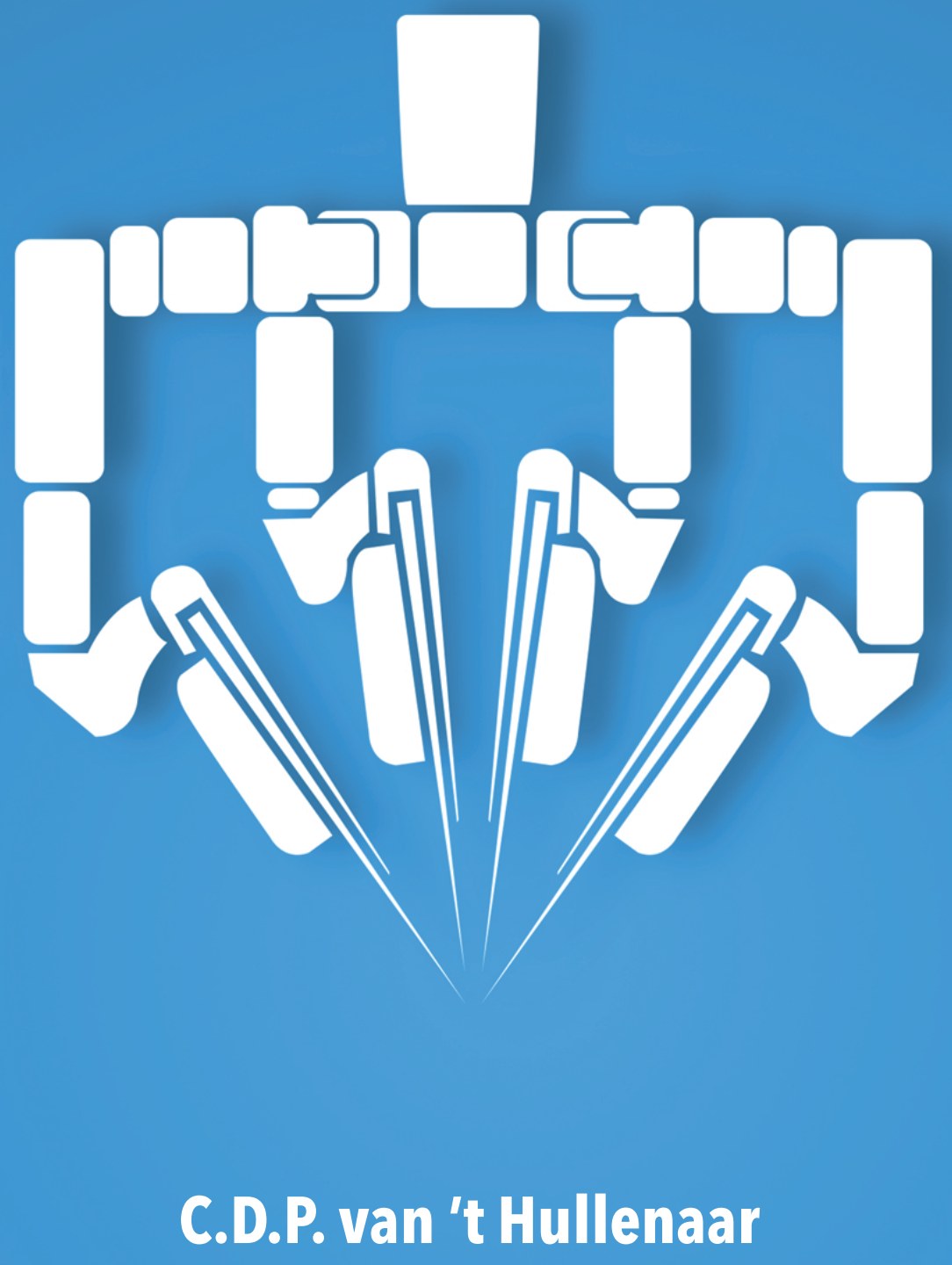





\title{
Ergonomic and safety insights in minimal invasive surgery
}

\author{
C.D.P. van 't Hullenaar
}


Ergonomic and safety insights in minimal invasive surgery

PhD thesis, University of Twente, Enschede, The Netherlands

Copyright (C) Cas van 't Hullenaar, Utrecht, 2018

All rights reserved. No part of this thesis may be reproduced or transmitted in any form or by any means without prior written permission from the author. The copyright of the papers that have been published or have been accepted for publication has been transferred to the respective journals.

Publication of this thesis was financially supported by:

Meander Medisch Centrum, ChipSoft, Erbe Nederland BV, Nederlandse Vereniging voor Endoscopische Chirurgie, Karl Storz Endoscopie Nederland BV, Two Hands Events, Medical Assistance International

Cover design: $\quad$ Okan Bastian, Utrecht

Printed by: $\quad$ GVO drukkers en vormgevers, Ede

ISBN: $\quad$ 978-90-365-4594-5 


\title{
ERGONOMIC AND SAFETY INSIGHTS IN MINIMAL INVASIVE SURGERY
}

\author{
PROEFSCHRIFT \\ ter verkrijging van \\ de graad van doctor aan de Universiteit Twente, \\ op gezag van de rector magnificus, \\ prof. dr. T.T.M. Palstra \\ volgens besluit van het College voor Promoties \\ in het openbaar te verdedigen \\ op donderdag 13 september 2018 om 16.45uur \\ door \\ Casper Dirk Paul van 't Hullenaar \\ geboren op 8 augustus 1980 \\ te Eindhoven
}


Promotor:

Co-promotor:

Datum:
Prof. dr. I.A.M.J. Broeders

Dr. J.P. Ruurda

Donderdag 13 september 2018

\section{Commissie samenstelling:}

Prof. dr. I.H.M. Borel Rinkes

Dr. M.J. van Det

Prof. dr. ir. H.J. Hermens

Dr. T. Horeman

Prof. dr. ir. H.F.J.M. Koopman

Prof. dr. W.J.H.J. Meijerink 


\section{Table of Contents}

Chapter 1 General introduction and outline of the thesis

\section{Part 1 Checklists in minimal invasive surgery}

Chapter 2 Checklists and time-out procedures in the operation theatre

C. van 't Hullenaar, E. Consten, I. Broeders

Chapter 24, Textbook of Endoscopic Surgery (in Dutch)

ISBN / ISSN 9789031350827, Bohn Stafleu van Loghum, 2009

Chapter 3 Implementation of a preoperative checklist in laparoscopic surgery

reduces errors and improves efficiency

C. van 't Hullenaar, A. Pomp, E. Consten, G. Dakin, I. Borel Rinkes,

I. Broeders

Research project performed at Weill Cornell Medical Center / NYP, New York City, USA

\section{Part 2 Ergonomics in minimal invasive surgery}

Chapter 4 Towards the ideal starting posture for the surgeon during endoscopic surgery: analysis of the optimal ergonomic laparoscopic position in a simulation environment

C. van 't Hullenaar, M van Alphen, M Hendriks, I. Broeders Research project performed at Laboratory of Biomechanical Engineering, Twente University, The Netherlands

Chapter 5 Ergonomics, user comfort and performance in standard and robotassisted laparoscopic surgery

R. van der Schatte Olivier, C. van 't Hullenaar, J. Ruurda, I. Broeders Surgical Endoscopy (2009) Jun; 23(6):1365-71.

Chapter 6 Ergonomic assessment of the da Vinci console in robot-assisted surgery C. van 't Hullenaar, B. Hermans, I. Broeders Innovative Surgical Sciences (ISS, 2017), Volume 2, Issue 2, Pages 97-104 
Chapter 7 The design of a console chair by addressing the ergonomic deficits in

robot assisted surgery

Research project performed at the Meander Medical Center,

Amersfoort, The Netherlands

H. Blankenvoort, C. van 't Hullenaar, I. Broeders

Derived from Part 2 of the Master graduation thesis of

H. Blankenvoort, Faculty of Industrial Design Engineering, Twente

University, The Netherlands

\section{Part 3 Advanced ergonomics in minimal invasive surgery}

Chapter 8 Validation of ergonomic instructions in robot-assisted surgery simulator training

C. van 't Hullenaar, A. Mertens, J. Ruurda, I. Broeders

Surgical Endoscopy (2018) May; 32(5):2533-2540.

Chapter 9 Ergonomic assessment of the first assistant during robot-assisted surgery

C. van 't Hullenaar, P. Bos, I. Broeders

Journal of Robotic Surgery (2018) Jul; 24.

DOI: 10.1007/s11701-018-0851-0 [Epub ahead of print]

Chapter 10 Ergonomics in hand-held and robot-assisted camera control:

a randomized controlled trial

P. Wijsman, L. Molenaar, C. van 't Hullenaar, S. van Vugt,

W. Bleeker, W. Draaisma, I. Broeders

General discussion and futūre perspectives

List of publications 
Chapter

General introduction and outline of the thesis 

For general surgeons the era of minimal invasive or laparoscopic surgery started in the early 1980s. It was then that the first series of laparoscopic cholecystectomies were performed in Germany. Towards the end of that decade, in 1989, Professor Perissat from Bordeaux, France, played the audience at the meeting of the Society of American Gastrointestinal and Endoscopic Surgeons (SAGES) a video of a laparoscopic procedure. This marked the beginning of a global revolution in surgery. Swathes of surgeons around the world began using the technique of laparoscopic surgery. Nowadays, millions of laparoscopic surgical procedures are performed annually.

Minimal invasive surgery has several major advantages over conventional open surgical procedures. Laparoscopic surgery limits scarring as skin incisions and abdominal wall incisions are much smaller in size. This leads to less tissue damage, less wound pain and a reduction in hospital stay. The introduction of minimal invasive surgery, however, posed surgical teams several challenges. It forced surgical team members to adopt different postures, changing their ergonomics. Most important, the target area where the surgery is performed is visualised on a monitor. This influences one's posture. Screen height and screen distance are identified as very important variables in the ergonomics of laparoscopic surgery. Many surgeons tend to place their screens too far away. This can trigger forward bending or leaning, which may cause problems to the cervical spine. Also, working with a screen that doesn't correspond with one's own trunk and arm-working axis, causes a surgeon to move his neck and trunk at unfavourable angles.

Several studies mapping the ergonomics of minimal invasive surgery were conducted towards the completion of this thesis. Several key elements are essential to creating a safe and healthy environment for both the patient, as well as the physician, prior to initiating an operational procedure. "Sufficient preparation is half the battle" is a proverb common to many languages. "Failing to prepare, is preparing to fail," is another phrase often used in this context. These proverbs certainly make sense when preparing for a surgical procedure. Checking the optimal conditions through a standardized checklist is an essential element in this respect. Suboptimal positioning of the patient on the operating table can generate many obstacles during a procedure. The same goes for incorrect positioning of hardware components and screens in the operating theatre.

This thesis is divided into three sections. Part one outlines the introduction of a surgical safety checklist to preparing for minimal invasive procedures. Part two discusses fundamental ergonomics in minimal invasive surgery. Several research projects were initiated to investigate what the best posture would be in an artificial environment. It also discusses the introduction of robot-assisted surgery and its implications to posture. Part three discusses advanced ergonomics and the impact of robot-assisted surgery (chapter 7-10).

Chapter 2 outlines the relevance and importance of checklists and time-out procedures. The 2000-2015 period saw increased attention given to reducing the number of surgical errors made in operating theatres across the globe. Many governmental and non-governmental organisations were involved in creating a safer and more efficient 
working environment inside the operating theatre. Safety issues often go hand in glove with the sound preparing of a certain task. This chapter will see us discuss the urge and rationalization underlying a surgical checklist. It will also present an example of such a list, specifically designed for use in minimal invasive surgery.

Chapter 3 looks into the effects of the implementation of a preoperative checklist. A large research project aimed at scoring surgical errors and disturbance in the operating theatre was conducted. Introduction of a checklist may take some effort and time, but can be very beneficial when objective values are considered. For instance, interruptions due to instrument-related issues are a critical concern during surgical procedures. They can cause time loss, annoyance and hindrance. The time needed for completing a full checklist was recorded and analysed. This chapter also addresses the effects on total surgical procedure time. Verification of hardware used to be a minor issue, but can checklists create an awareness among surgeons that they are responsible for the materials they work with? Time slots, inspections and validation of instrument trays were also scored in this study.

Chapter 4 contains a laboratory study aimed at finding the optimal posture a laparoscopic surgeon should adopt. All regular operating theatre obstacles were removed in an infrared laboratory setting. The positions of the shoulders, elbow and trunk was recorded. The study also included a thorough analysis of the view-instrument axis, as well as the ideal neck position. Chapter 4 lists all angles, allowing them to serve as a benchmark for future research.

Chapter 5 describes the differences in laparoscopic versus robot-assisted surgery. It assessed several parameters. This was done using an ambulatory monitoring system in order to objectively measure the physiological impact of both types of surgery. Also, questionnaires were taken and performance scores were calculated to make an overall comparison to see whether any differences could be found between the groups.

Observations, research and preferences of surgeons learned that working in a console during robot-assisted surgery showed benefits to ergonomics compared to laparoscopic surgery. However, detriments to posture were also observed during the surgical activities when working in the console. One of the major drawbacks is the forward-bending, or leaning, position required to steer the two telemanipulation handles inside the console. This is why the quest for optimal ergonomics continues in Chapter 6. This chapter features a thorough analysis of the surgeon's sitting position inside the console. Calculations are made to determine angles in specific joints using a 2D geometric model. Special attention in this respect is given to the cervical spine, as many surgeons experience neck pains. It also addresses several of the console's limitations.

At this moment in time, the console of the da Vinci robot system does not come with an official chair. In order to determine the surgeon's optimal sitting position, a comprehensive research project was set up. Chapter 7 describes how the design of a special console chair came about. This was a project undertaken in collaboration with a 
medical chair manufacturer. It aimed to remedy some of the ergonomic deficits to the current console set-up.

Part three of this thesis contains an in-depth analysis of the ergonomics of robotassisted surgery. It starts off with Chapter 8 , which sets out how a training program was set up and validated. In this training program, a brief explanation of the ergonomics was combined with on-site training exercises and additional explanation. Participants performed two sets of training exercises on the da Vinci skills simulator. Performance scores were calculated, and questionnaires were registered. This group was compared to another group which received only standard instructions.

Chapter 9 highlights an entirely different aspect of robot-assisted surgery. While the instruments on the tips of the robot arms are controlled by the surgeon, a first assistant or scrub nurse will always be in attendance, standing next to the patient near the operating table. This assistant plays a vital role in all surgical procedures. Instruments need to be changed, needles substituted, and tackers and staplers will occasionally need to be fired. Some procedures will quite often also require the use of a suction device. A physician or nurse assisting on such a procedure, will have to deal with the robot's severe arm movements. On top of that, the robot arms may interfere and cause hindrance to the person assisting the procedure. To this end a research project was conducted into the first assistant's ergonomics in robot-assisted surgery. This project saw questionnaires taken and objective operating theatre measurements scored by an observer. The chapter concludes with several recommendations for the surgical team.

The last chapter, Chapter 10 describes the role of the robotic camera holder in laparoscopic surgery. The goal of this project was to find out whether such a device had a serious impact on the dynamics and ergonomics of a minimal invasive surgical procedure. Two groups were created by a randomized trial. One group performed all procedures with the help of an assistant steering the camera, in the other group the robotic camera holder was set in place. Both groups were scored by measuring their RULA scores, derived from photographs taken in the operating theatre. Surgeons and their assistants also completed questionnaires. These data allowed the effect of a robotic camera holder on ergonomics to be evaluated. 



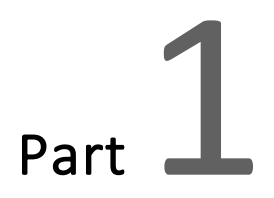

Checklists in minimal invasive surgery 



\section{Chapter}

\section{Checklists and time-out procedures in the operating theatre}

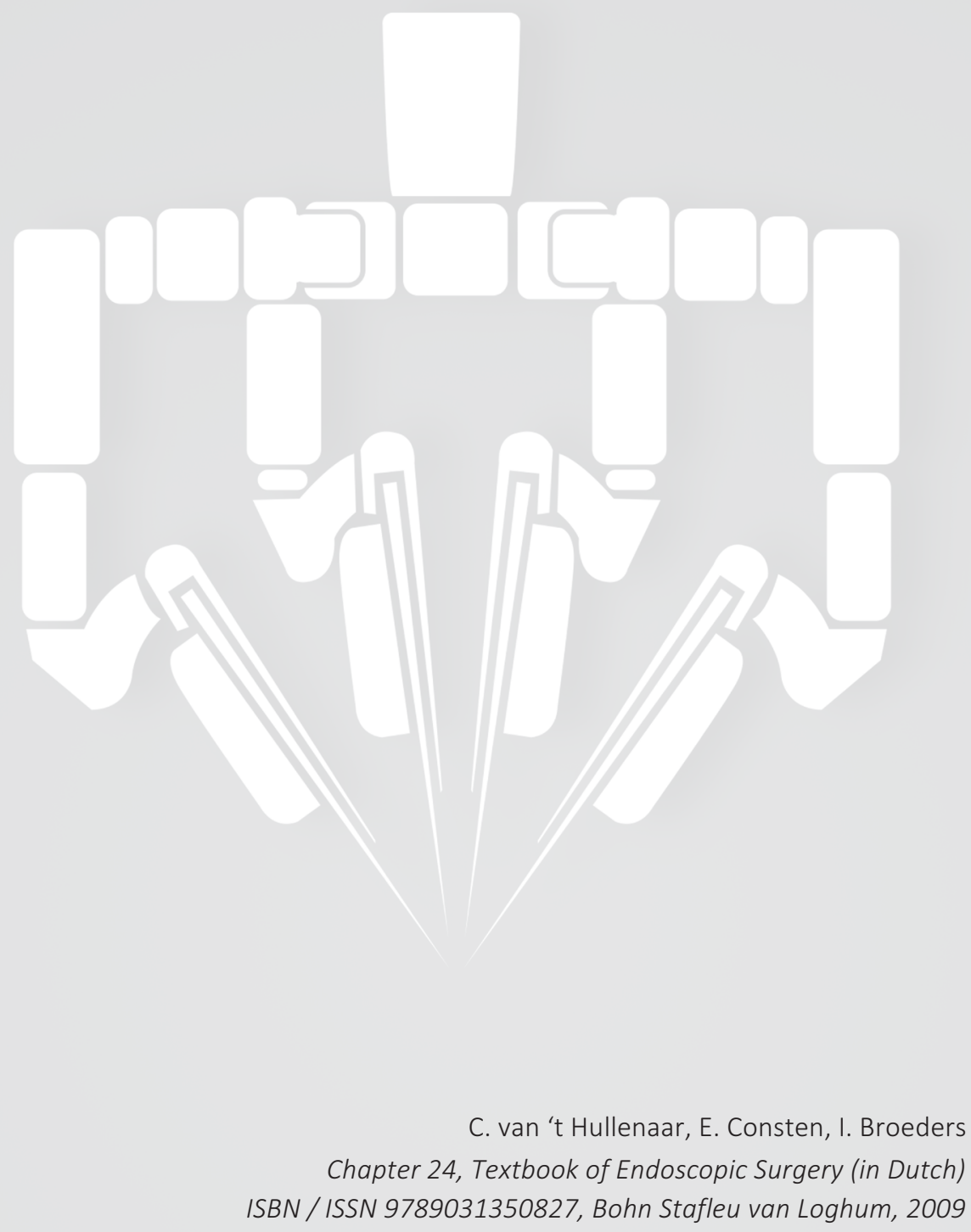





\section{Introduction}

Endoscopic surgery took a leap forward following the introduction of the laparoscopic cholecystectomy in 1987. The spread of this new technique was rapid and uncoordinated. The technique's 'point of no return' had been reached before its actual added value had even been confirmed in large randomized studies. The main focus in endoscopic surgery over the past twenty years has been on technological innovation and the expansion of indications. Attention to safety and education came second in this respect. This is an understandable phenomenon in a period of profound change in the applied techniques. However, definitive anchoring of a technique requires a professional approach to education and care for patient safety and efficiency.

The introduction of endoscopic techniques runs parallel with major changes in the professional attitude of surgeons. These new treatment methods have therefore been chosen as the benchmark for change processes in medical treatments. One could say that the classic 'master-apprentice' training model is disappearing. Medical professionals also no longer enjoy full autonomy. Treatment outcomes are no longer confidential and hospitals are assessed on their performance, both by the Dutch Health Care Inspection as well as by various media. For example, in 2007 the Dutch Health Care Inspection detected a disproportionate number of serious calamities in surgical procedures where endoscopic techniques were applied. This led to a national study involving surgeons, gynaecologists, care managers and departmental heads of the central sterilization departments. The Inspection found that safety for the patient in endoscopic surgery was insufficiently warranted due to gaps in training, certification, hospital policy and equipment checks. [1]

A package of far-reaching measures was announced that has been gradually introduced since March 2008. The Dutch Health Care Inspection has placed responsibility for restructuring with the professional societies as well as with individual hospitals and medical doctors.

\section{Training and certification}

All surgeons are primarily responsible for defining training requirements for their own colleagues. A surgeon is also responsible for the certification of specific interventions and procedures. Meeting these requirements not only requires a great deal of work, but above all requires the realisation of a complete change in the working culture of surgeons. Almost unrestricted freedom to act, without any compulsory professional testing, should make way for structured training with fixed benchmarks. Surgeons should be certified during and after their training for certain types of procedures, e.g. endoscopic surgery of the colon, and record their results. Such an approach can be formulated clearly on paper, but will in practice require a far-reaching change in thinking and acting. It is nevertheless evident to medical societies that adapting to current social standards is unavoidable. The training to become a surgeon is now undergoing radical restructuring. The best-known example is the 'SCHERP training plan' which has been incorporated into 
surgical training. [2] The training plan makes it results of the training process available to trainers via an online digital training portfolio. The training qualifications for endoscopic surgery are also structured at national level. Clear requirements that incorporate knowledge and skills in endoscopic surgery are nowadays compulsory for all surgical residents. Surgical interventions are categorized, recorded and protocols are implemented. Best practice guidelines are published by surgical societies. The optimal techniques for performing a laparoscopic appendectomy and laparoscopic cholecystectomy have been included in the guidelines of the Dutch Association for Surgery (NVVH). Guidelines for colon surgery are also being drawn up. [3]

The Dutch Society of Endoscopic Surgeons (NVEC), as well as different taskforces from the departments of Gynaecology (WGE) and Urology (SWEN) are formulating educational and accreditation requirements for complex surgical procedures. In years to come, such demands will become universal.

Every surgeon will have to apply to certification and registration. Outcome parameters will be part of the public domain. Hospitals must take responsibility regarding multidisciplinary restructuring of endoscopic procedures. Core components such as safety of equipment and instruments, as well as optimization of the pre- and postoperative course, must be considered here. An important part of structuring the operative course applies to all surgical procedures. This chapter discusses the standardized methods for improving safety and efficiency. It only looks at the phase between the moment a patient arrives in the operating theatre and the moment he leaves.

\section{Preoperative counselling}

Prior to surgery, patient related information is collected by a GP, a referring doctor, a surgeon, an anaesthesiologist and a surgical planner. This information is usually recorded to various documents. At the time of the procedure, all this information and data are needed to create a safe and efficient workflow and must be immediately available.

The Dutch Health Care Inspection has carried out a survey of the organisation of preand postoperative care in Dutch hospitals and has documented the results of its survey in two reports, which were published in 2007 and 2008.[1] The Inspection found structuring of these processes lacking and has set out requirements for improvement in its reports. The societies also address the need for improvement. Endoscopic surgery is regularly used as an example here, since the use of a series of electronic, or computercontrolled, instruments makes this type of intervention susceptible to operative problems. Research by Verdaasdonk et al. showed disruptions to occur in $70 \%$ of endoscopic procedures that lacked a structured preparation.[4] 


\section{Checklists and time-out procedures}

Many high-tech industries employ checklists as part of their working routine in an attempt to allow them to enjoy the best possible preparation. Obvious examples of this can be found in the aviation industry and at nuclear power plants. Standardized checklists are an integrated part of the daily working routine there. In anaesthesiology, the use of checklists has also been common practice for quite some time. Doctor Hart et al. published an article in 2005 on the merits of such a list.[5]

The introduction and implementation of a time-out procedure combined with a surgical checklist will soon be incorporated into the operative course at all hospitals. This demands a change in attitude: one may expect to be met with resistance if attempting to introduce checklists that are time-consuming and require a great deal of administrative chores. Kalkman showed that checklists that are too complex, render themselves ineffective.[6] Whether a checklist is effective or not is dependent on the manner in which action is taken pursuant to the results they generate. Applying a checklist without the correct specific attention or without properly following up on the results it generates, renders it a counter-productive instrument. That is why it is of vital importance to check all essential information using a brief and user-friendly checklist. This will allow the preparation of surgical procedures to be optimized. Hospitals will need to appoint people who are involved in surgical care to their executive boards. Subsequently, all sources of information are then subjected to analysis, with the collection of information integrated into the checklist. This prevents major changes to effective administrative processes and will lead to a better acceptance of the checklists.

\section{Perioperative checklist}

A perioperative checklist for endoscopic procedures focuses on the following six components:

1. Identification of the patient and the intervention, patient-related risk factors;

2. Choice and availability of instruments;

3. Check of required hardware;

4. Positioning of the patient;

5. Configuration of the work environment;

6. Postoperative instructions.

These six components should contain the following elements:

1. Identification of the patient and the intervention, patient-related risk factors

The patient's identity is recorded by direct communication. The patient's wristband is checked, especially on patients who are being ventilated, or unable to communicate. The patient data in the electronic patient file are checked against both the information obtained orally and the data on the wristband. The nature of the planned surgery is confirmed by the patient and is recorded in the file, as well as the side body the procedure 
is set to be performed. Relevant risk factors are verified for correspondence with the information contained in the patient file. Special consideration is to be given to cardiac and pulmonary abnormalities, clotting disorders, liver and kidney dysfunction, diabetes, neurological abnormalities and known allergies to medication, latex or dressings. The medication list is reviewed and the information about specific medication, e.g. antibiotics or corticosteroids is also checked.

\section{Choice and availability of instruments}

The availability of the standard laparoscopic instruments is verified. The request for specific requirements is discussed. All necessary equipment should be available before the patient is administered his anaesthetic.

\section{Check of required hardware}

The scrub nurse programmes the required hardware according to an established protocol. The responsible surgeon then performs a second check of all necessary hardware for their functioning and settings. As he is responsible for the maintenance status of all the individual components, the surgeon should always be informed of any technical issues.

\section{Positioning of the patient}

The patient is positioned either in accordance with the applicable protocol, or pursuant to the surgeon's additional instructions. Probes and catheters are inserted where necessary. Planned adjustments of the table position, such as tilt and rotation, are tested. The positioning of protective measures against shifting - such as the vacuum mattress and support bands and pillows - is checked. The anaesthesiologist performs a final check to reduce the risk of thrombosis, decubitus, and nerve or joint injury. The surgeon then conducts a final check of the desired positioning of the team around the operating table and the accessibility of the working area.

\section{Configuration of the work environment}

The instrument tables and required hardware are set up according to protocol, usually accompanied by additional instructions from the surgeon. The screens are set to their correct positions in relation to the desired work axis. Should the screens be expected to require repositioning during the procedure, the feasibility of such manoeuvring is also checked. Should the positioning of the screens obstruct the sterile dressings, the screens are then moved perpendicularly in the direction of the ceiling. This allows them to be easily repositioned at the start of the procedure. Foot pedals are correctly placed, and the working environment is checked for disturbing elements, e.g. any unrequired tables, chairs or disruptive cables.

\section{Postoperative instructions}

Following the procedure, postoperative pain schedules are composed. Specific medication, fluid management, blood loss, catheters, drains and probes, mobilization instructions and diet prescriptions are documented. Providing postoperative information to the patient's family is recorded to the checklist. Video footage and data obtained during the procedure are selected and secured to the electronic patient file. 


\section{Time-out procedures}

There are three specific time-out procedures that can be incorporated in respect of the six items mentioned in the foregoing,

a. A time-out after components 1 and 2. The parties involved are the patient (component 1), the surgeon, the anaesthesiologist and the scrub nurse. The patient will only be administered the anaesthetic if these two components have been successfully satisfied.

b. A time-out after components 3,4 and 5. The parties involved are the patient (component 1), the surgeon, the nurse or anaesthesiologist and the scrub nurse. Once all five components have been addressed, draping can then start and the surgeon can leave for scrubbing.

c. Time-out at the end of the procedure, before the patient is brought to the recovery room. The parties involved are the surgeon and the anaesthesiologist. Component 6 is addressed in a so called 'sign-out procedure'.

This can be used as a basis for drawing up an effective and compact checklist. Time-out procedures should be designed in such a way that they are easy to use and minimalize paperwork.

\section{Closing remarks}

As mentioned, acceptance and a meaningful use of surgical checklists and time-out procedures depends on a simple structure. Alongside to this is advanced digitalisation of hospital administration and patient files. The use of many forms that accompany the patient, has become obsolete and should be avoided. The checklist largely consists of questions that are answered during preoperative visits. Details that are not related to patient data are often suitable for incorporating into protocols. Any changes should be implemented at the outpatient clinic to allow deviation from protocol to be anticipated. This could occur in cases where extra instruments might be needed, or if serious abnormalities to the patient's constitution have been found. The completion of the checklist is based on different preoperative assessments. This implies that the checklist should be accessible from a range of digital locations.

Ease of access is key in this regard. Surgeons and nurses cannot spend their precious time waiting to be granted access, or having to undergo complex authorization procedures. Current practice mainly relies on the use of passwords. In the near future, quicker alternatives will help facilitate the acceptance of checklists. This may take the form of finger pattern recognition or iris scans, or Radio Frequency Identification tags (RFID - also used in product recognition). The use of RFID, however, remains a point of debate. In their article in the Journal of the American Medical Association (JAMA), researchers at the Academic Medical Centre (AMC) in Amsterdam and the Dutch Organization for Applied Scientific Research (TNO) in Delft concluded that RFID tags and 
readers emit electromagnetic fields that interfere with critical medical equipment such as respirators, defibrillators, pacemakers and infusion pumps.[7]

Doctors should be able to gain access to the hospital computer system from any workstation. One-time identification should provide direct access to all sources the user has been authorised to access. The user's computer offers him a desktop matching his needs, and which includes a simple representation of the most frequently used sources. The checklist programme should then be able to be opened with a single click and should only require input of the unique patient data. In the future this process may also be automated at the operating theatre through the detection tag the patient in question would be wearing. This is how a comprehensive digital hospital information system could contribute to both a simple and efficient application of checklists and an optimal registration of patient-related information. 


\section{Sources}

1. Rapport 'Risico's minimaal invasieve chirurgie onderschat, kwaliteitssysteem voor laparoscopische operaties ontbreekt.' Thematisch rapport, 2007. Inspectie voor de Volksgezondheid. https://www.igj.nl/documenten/rapporten/2007/11/15/risicos-minimaal-invasieve-chirurgie-onderschat

2. Opleidingsplan Heelkunde, 2007 https://www.knmg.nl/opleiding-herregistratie-carriere/cgs/regelgeving/ huidige-regelgeving-per-specialismeprofiel/heelkunde.htm

3. Richtlijn colorectaal carcinoom, 2014 https://richtlijnendatabase.nl/

4. Verdaasdonk EG, Stassen LP, Hoffmann WE, Elst M van der, Dankelman J. Can a structured checklist prevent problems with endoscopic equipment? Surgical Endoscopy 2008;22:2238-43.

5. Hart EM, Owen H. Errors and omissions in anaesthesia: a pilot study using a pilot's checklist. Anesth Analg 2005;101:246-50.

6. Kalkman CJ. Veiliger OK: gemakkelijker gezegd dan gedaan. Nederlands Tijdschrift voor Geneeskunde 2008;152: 2270-72.

7. Electromagnetic Interference From Radio Frequency Identification Inducing Potentially Hazardous Incidents in Critical Care Medical Equipment. Remko van der Togt, MSc; Erik Jan van Lieshout, MD; Reinout Hensbroek, MSc; E. Beinat, PhD; J. M. Binnekade, PhD; P. J. M. Bakker, MD, PhD. JAMA. 2008;299(24):2884-2890. doi:10.1001/jama.299.24.2884 



\section{Chapter}

\section{Implementation of a preoperative checklist in laparoscopic surgery reduces errors and improves efficiency}

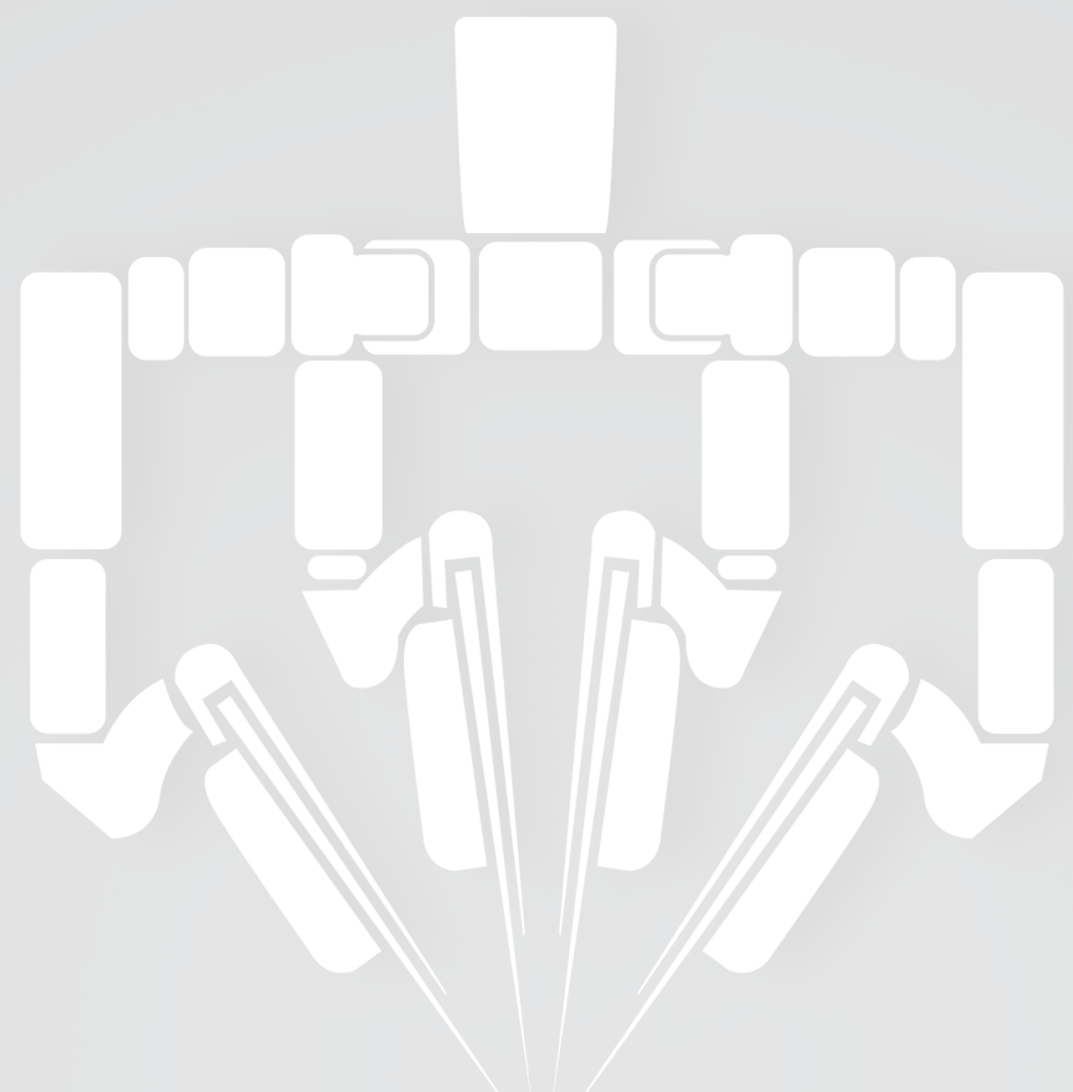

C. van 't Hullenaar, A. Pomp, E. Consten, G. Dakin, I. Borel Rinkes, I. Broeders Research project performed at Weill Cornell Medical Center / NYP, New York City, USA 


\begin{abstract}
Background: Laparoscopic surgery has increased the complexity of the operating room (OR) environment and thereby enlarged the potential for errors and technology-related problems. The potential for errors may be minimized through the use of preoperative checklists that help standardize the work process. A structured and thorough preparation of the $O R$ is likely to reduce preparation times and decrease the number of technical problems that may surface during surgical procedures. The goal of this study is to investigate whether working with a preoperative checklist could help improve efficiency in laparoscopic procedures.
\end{abstract}

Methods: 106 consecutive laparoscopic procedures were observed and analysed by a single observer. Five surgeons, each enjoying vast experience in laparoscopic surgery, participated in this study: three worked without a checklist, two worked with a checklist. A five-step checklist - which included verification of patient characteristics, instruments, hardware and software settings, the patient's position, and the position of hardware components - was applied in every procedure. Key time intervals and errors were recorded during all procedures.

Results: The patient identification process improved by $19 \%$ upon introduction of the checklist. Instrument verification improved from $42 \%$ to $89 \%$. Patient re-positioning was required in $32 \%$ of the non-checklist cases, compared to $0 \%$ in checklist cases. Cases working with the checklist saw time-slots reduced. Preparation time was brought down from 4.6 to 3.5 minutes. Errors and potential side-effects were minimized.

Conclusion: Implementation of a preoperative checklist in laparoscopic surgery enhances efficiency and safety. These promising results emphasise the need for general implementation of preoperative checklists in this field of surgery. 


\section{Introduction}

The widespread use of laparoscopic techniques in the operating theatre has vastly increased the complexity of the surgical process. Endoscopes, cameras, light sources, insufflators, energy sources, multimedia devices, and a vast array of complex gastrointestinal staplers have all been introduced to the modern laparoscopic operating room (OR). Laparoscopic surgery requires a set-up featuring multiple high-tech hardware components located in close vicinity of the sterile field. This high-tech environment is more susceptible to technical errors or malfunctioning than those of most conventional surgical procedures $[1,5]$. Standardisation and a structured preparation of the OR may reduce the number of technical errors. As safety and efficiency are key issues in today's healthcare management, the quest to find tools for improving safety and efficiency remains an ongoing endeavour. Checklists are a commonly-used tool for reducing technical problems in complex working environments [1-10, 28]. Currently, the use of checklists is rarely applied to surgeries. The aim of this prospective cohort study was to analyse whether optimal preoperative preparation through the use of a checklist facilitates efficiency in laparoscopic surgery and could potentially reduce the number of perioperative problems.

\section{Materials \& Methods}

\section{Research setting}

The study was performed in two dedicated laparoscopic operating theatres at Weill Cornell Medical College / New York Presbyterian Hospital (New York, NY, USA). Five members of surgical staff there participated in this project. Over a period of four months, 106 consecutive laparoscopic operations were recorded by a single observer (CvtH). Three surgeons performed their procedures without a preoperative checklist. Two surgeons applied the checklist every time they started a laparoscopic procedure.

Five items were identified as essential to the preparation of laparoscopic procedures: 1) patient identification (including case-related information and procedure verification), 2) instrument selection, 3) patient positioning, 4) hard- and software settings and 5) hardware component positioning. These five important items were derived from previous studies and determined by an expert panel [17-21, 24, 26].

\section{Data collection}

The collection of data was done using two forms: an observation form completed by the researcher, and a checklist form completed by the surgeon. The checklist form was filled out by the surgeon before the start of the procedure. This began with the checking of the patient's characteristics. The surgeon had to be sure that identification of the patient and verification of the procedure was done correctly. Also, cardiopulmonary co-morbidity and the use of anticoagulants were acknowledged. On the instrument section of the form, the surgeon was able to verify and cross check all the instruments needed to perform the procedure. The hardware \& software check was designed to verify all technical devices, 
from the electronic patient file to the energy source. The surgeons checked the presence of the items and confirmed their proper functioning. The monitors were positioned and recordings with regard to the eye-to-screen distance were made. An optimal ergonomic eye-to-screen distance is approximately three feet [31]. The fourth item contained information regarding patient position and additional items such as the application of an intermittent compression device for the prophylaxis of venous thromboembolism, placement of a Foley catheter, and placement of a cautery grounding pad. The researcher was present at every single procedure and filled out a detailed observation form. Timeslots were accurately recorded in a timetable on the observation form. Two relevant time-slots for this study were 'Preparation Time' and 'Interval Time'. 'Preparation Time' was defined as the timeframe between the moment the surgeon starts with the preparation for the procedure and the moment he is ready to start draping the patient. 'Interval Time' was defined as the period of time between draping the patient and the moment of the first incision.

Patient, surgical team and procedure characteristics, as well as instrument-related, positioning-related, hard- and software-related items were also recorded during the case. Situations during the procedures that could potentially lead to complications or accidents were extracted from previous research [5] and subsequently determined by expert opinion. Potentially harmful situations were recorded on the 'event list' of the observation form.

\section{Results}

\section{Patient identification and characteristics}

Without the checklist, personal identification of the patient by the surgeon was performed in $80 \%$ of cases. The surgeons who worked with the checklist identified all their patients and procedures as part of the protocol and enjoyed $98 \%$ compliance (Table 1). Surgeons working without the checklist, verified the patients' risk factors in $79 \%$ of cases. The surgeons who used the checklist verified all risk factors regarding the patient's co-morbidity, anti-coagulative medication and allergies in all cases. None of these comorbidity recordings led to any change in operative policy, or delay in surgery.

\section{Instruments}

Surgeons working without the checklist, checked for the presence of required surgical instruments and tools before draping in $42 \%$ of cases (Table 1). A significant increase in this area was witnessed in the checklist group: in $89 \%$ of procedures, the instrument table was verified through performing a thorough inspection of instruments $(p=0.02)$. The instrument check in the checklist group resulted in a $75 \%$ reduction in intraoperative interruptions. The number of times that missing instruments had to be obtained from storage locations dropped from 28 to 7 events. 
Table 1. Adherence to the selected tasks

\begin{tabular}{llll}
\hline & Checklist group $(\mathrm{n}=53)$ & $\begin{array}{l}\text { Non-checklist group } \\
(\mathrm{n}=53)\end{array}$ & P value \\
\hline Identification performed & $98 \%(52 / 53)$ & $79 \%(42 / 53)$ & 0.01 \\
Instruments checked & $89 \%(47 / 53)$ & $42 \%(22 / 53)$ & 0.02 \\
$\begin{array}{l}\text { Interruptions due to instrument related } \\
\text { problems }\end{array}$ & $13 \%(7 / 53)$ & $53 \%(28 / 53)$ & 0.01 \\
Patient position checked & $100 \%(53 / 53)$ & $68 \%(36 / 53)$ & 0.01 \\
Hardware verification & $92 \%(49 / 53)$ & $13 \%(7 / 53)$ & $<0.01$ \\
\hline
\end{tabular}

The percentages are displayed as the number of times a check or task was performed correctly

\section{Positioning of the patient}

During most procedures, the surgeon was actively engaged in the process of adjusting the patient's position on the operating table. In all cases (100\%) of the checklist group, the surgeon performed the positioning of the patient (Table 1). In cases where surgeons did not employ a checklist, patient positioning was performed by the surgeon in $68 \%$ of cases $(p=0.01)$. In instances where the remaining members of the OR team performed the positioning of the patient, this resulted in a satisfactory or acceptable position in $76 \%$ of cases (13 out of 17 cases). In four procedures (24\%), adjustments of the patient's position had to be made by the surgeon. $100 \%$ of patients of patients in either group were treated with a sequential compression device. Placement of a Foley catheter and placement of a cautery grounding pad were part of the routine preoperative work-up and were consequently performed in all patients in both groups. Contrary to surgical protocol, placement of a Foley catheter was not performed in one patient of the non-checklist group (Table 2). Administration of perioperative antibiotics was performed by the anaesthesiologist, often with instructions or guidelines from surgical staff. Specific antibiotic statements were therefore excluded from the checklist form. Obvious errors regarding the administration of antibiotics were nevertheless registered on the event form (Table 2).

\section{Hardware and ergonomics}

As displayed in Table 1, a check of the hardware components of the laparoscopic video and hardware tower ('hardware verification') was incidentally performed in the nonchecklist group (13\%). With the use of the checklist, a serious check of hardware components (such as insufflator, Xenon light, camera head, etc.) was performed in $92 \%$ of cases $(p<0.01)$. Another distinct difference was the involvement of the surgeon in the positioning of the monitor. When working with a checklist, surgeons positioned the monitor preoperatively in $85 \%$ (45/53) of cases. In the procedures where no checklist was applied, this was done in only $23 \%(12 / 53)$ of procedures $(p<0.01)$.

In the checklist group, the ergonomically ideal eye-to-screen distance of three feet was met in 91\% (48/53) of cases. The three surgeons who did not work with the checklist, 
performed 35 out of 53 (66\%) procedures with an ergonomically suboptimal eye-monitor distance greater than three feet [31] (Table 2).

Table 2. Serious events reported

\begin{tabular}{lll}
\hline Types of events & $\begin{array}{l}\text { No. of times } \\
\text { errors occurred } \\
\text { checklist group }(n=53)\end{array}$ & $\begin{array}{l}\text { No. of times } \\
\text { errors occurred } \\
\text { non-checklist group }(n=53)\end{array}$ \\
\hline $\begin{array}{l}\text { OG tube incorrectly placed by anaesthesia } \\
\text { technician }\end{array}$ & 0 & 2 \\
Surgical instrument related error & 1 & 3 \\
Foley catheter not placed & 0 & 1 \\
Suboptimal eye-monitor distance $(>3 \mathrm{ft})$ & 5 & 35 \\
Hardware malfunctioning & 1 & 3 \\
Antibiotics/medication error & 1 & 2 \\
Total no. of events & 8 & 46 \\
\hline
\end{tabular}

The numbers displayed in the table are the recorded events for the two groups.

\section{Time-slots}

With the use of the checklist, a reduction was documented in both time-slots (Table 3). Without the use of a checklist, mean 'Preparation Time' for laparoscopic procedures was 23 minutes. In the checklist group, mean 'Preparation Time' was brought down to 21 minutes (NS, $p=0.15$ ). 'Interval Time' went down from 4.6 to 3.5 minutes $(p=0.02)$. The average time for completing the checklist was 4.1 minutes. A considerable part of the checklist could be filled in during the anaesthesia preparation time.

Table 3. Time allocation

\begin{tabular}{lll}
\hline & \multicolumn{2}{l}{ Time slots (average of all procedures) } \\
\hline Group 1 Without checklist & Preparation Time & Interval Time \\
Group 2 With checklist & $23 \mathrm{~min}$. & $4.6 \mathrm{~min}$. \\
P value & $21 \mathrm{~min}$. & $3.5 \mathrm{~min}$. \\
\hline
\end{tabular}

\section{Surgical Pause / Time-Out Procedure}

In accordance with hospital regulations, a surgical pause was performed prior to the start of every procedure. The purpose of this Time-Out Procedure was to conduct a final check as to whether the scheduled procedure involved the correct patient, the correct procedure, the correct operative site and the verification of antibiotic administration. Furthermore, all relevant documents and necessary equipment had to be present in the operating room. During this research project, the surgical pause was performed following the administration of general anaesthesia and the prepping and draping of the patient. The patient's name, hospital identification number and type of procedure were verified in all cases (100\%) of both groups. Double-checking of allergies and the administration of 
intravenous antibiotics were checked in 96\% (52/53) of all checklist procedures. In nonchecklist procedures, this was done in 19 out of 53 cases (36\%).

\section{Events}

Any near accidents, serious errors and potential adverse events were recorded on all procedures. A total of 54 potentially serious adverse events were recorded. Most problems occurred with the optimal positioning of the monitor. None of the errors caused any direct harm to the patient. Eleven errors other than monitor problems were observed in the non-checklist group, with three recorded in the checklist group. Table 2 contains a list of these events and details. In most incidents, communication issues were essential. These are mentioned separately. Most adverse events occurred due to inadequate teamwork and communication or to verification systems not being executed properly.

\section{Discussion}

Advanced technology in the field of surgery has increased the demand for doctors to cope with high-tech working environments. Laparoscopic procedures depend heavily on a variety of complex hardware and software applications. While the amount and complexity of equipment puts demands on the working environment and operating room staff, surprisingly little attention is paid to processing control, safety, and efficiency.

This study aimed to assess whether the use of a preoperative checklist could increase safety and efficiency. Five important items in laparoscopic surgery were addressed. In all items, promising results were demonstrated when the checklist was applied. Patientrelated items were accurately verified and potential risks factors identified. Devices and instruments were carefully checked and preparation was carried out more accurately. This led to fewer disruptions, consequently bringing down the number of potentially stressful situations. A better positioning of the hardware components was demonstrated and this resulted in improved ergonomics and monitor positions. Time-slots decreased significantly and important safety items were verified using the checklist.

Previous studies reported frequent malfunctioning of technical equipment and emphasised the desirability of improvements to, and standardization of, equipment in combination with the use of a preoperative checklist $[5,30]$. Although an extensive preparation of surgical procedures would appear essential to achieving optimal efficiency and safety, only a very limited number of studies undertook to investigate this [27-29].

Hart et al. published a study where a checklist had been designed for anaesthesiologists as they prepared prior to administering general anaesthesia [4]. This study pointed out that several important checks were denied when the preparation was performed with information and knowledge obtained from the practitioner's memory and experience. Most of the participating anaesthesiologists who participated in the study found the checklist a useful instrument and believed that it could improve patient 
safety [4]. Similar to anaesthesiology procedures, laparoscopic procedures are highly dependent on working with complex equipment. A great variety of instruments and hardware components such as cameras, monitors, insufflators, etc. has transformed the laparoscopic surgeon into a specialist dependent on high-tech equipment and therefore also subjected to the effects of their malfunctioning or non-presence [7].

Earlier studies have also found several factors that could lead to more efficient and safer surgical procedures. Working with a harmonious team in an established environment under similar conditions has proven effective in reducing the numbers of errors $[10,25]$. Furthermore, studies where basic checklists were incorporated in the surgical working process have shown promising results $[28,29]$. The application of an extensive preoperative checklist in laparoscopic surgery has never been described in any earlier publication. A recent study by Arora et al. [33] found several key stressors in surgical procedures. Equipment problems, technical problems and patient-related problems were all found to be highly stressful events during surgical procedures. The 55 procedures observed, showed a high frequency of occurrence for these problems.

As far as the limits to this study are concerned, it should first be noted that determining to what extent the awareness of the surgeons who were employing the checklist contributed to our positive results was not easy. One could state that some of the findings could be closely related to an increased awareness for potential errors. The increased awareness might be just as vital to safety matters as the actual process of verifying items using a checklist. Second, no patient outcome analyses were performed. The ultimate goal when implementing a checklist in surgery is to improve patient outcome. We gathered no specific data on this matter, nor was a follow-up of patients after surgery part of the study's protocol. The third item to address is the intraobserver variability. One researcher had been present at all procedures to document findings. The presence of a second researcher might have helped to improve the validity of outcomes. Finally, one could argue that the reduction of time slots could be due to several factors other than the checklist. Also, one may well question the clinical relevance of a decrease in time slots by 30 seconds and 2 minutes, respectively. The last remark that has to be addressed is the fact that less than $100 \%$ compliance was recorded in the checklist group. In an ideal situation, every checklist item would be marked, with any changes immediately implemented in case of any suboptimal situation.

No doubt the complexity of the operating room working environment will continue to evolve. A surgeon's duty remains the optimal attention for patient safety. A checklist appears to be a simple and helpful tool to increase awareness on critical patient issues and it helps to decrease hard-and software-related issues. The implementation of a checklist could potentially also abolish many moments of discomfort and frustration related to laparoscopic equipment and operation room ergonomics. Patients, surgeons and operating room staff are likely to profit from standardised safety procedures, since they have proven their merits in complex transport and industrial processes. ${ }^{32}$ 
The promising results of this study have inspired us and we will continue to conduct research projects that award a prominent role to preoperative checklists. Creating a digital format of a checklist that can be implemented in today's computer-driven laparoscopic operating rooms, is a challenge for the near future. A user-friendly digital checklist application will enable the swift and accurate preparation of surgical procedures. Such an application could be implemented into hospital roadmaps and digital medical charts, in order to guide patients through the surgical process in a friendly, safe and efficient manner. 


\section{References}

1. Helmreich RL. On error management: lessons from aviation. BMJ 2000; 320(7237):781-785.

2. Leape LL, Berwick DM, Bates DW. What practices will most improve safety? Evidence-based medicine meets patient safety. JAMA 2002; 288(4):501-507.

3. Helmreich RL, Davies JM. Culture, threat, and error: lessons from aviation. Can J Anesth 2004; 51(suppl_1):R1.

4. Hart EM, Owen H. Errors and omissions in anesthesia: a pilot study using a pilot's checklist. Anesth Analg 2005; 101(1):246-50, table.

5. Verdaasdonk EG, Stassen LP, van der EM, Karsten TM, Dankelman J. Problems with technical equipment during laparoscopic surgery. An observational study. Surg Endosc 2007; 21(2):275-279.

6. Calland JF, Guerlain S, Adams RB, Tribble CG, Foley E, Chekan EG. A systems approach to surgical safety. Surg Endosc 2002; 16(6):1005-1014.

7. Berci G. How new technology affects practice and patient safety. J Am Assoc Gynecol Laparosc 1997; 4(4):419-421.

8. Berci G, Phillips EH, Fujita F. The operating room of the future: what, when and why? Surg Endosc 2004; 18(1):1-5.

9. Greenberg CC, Roth EM, Sheridan TB et al. Making the operating room of the future safer. Am Surg 2006; 72(11):1102-1108.

10. Vincent C, Moorthy K, Sarker SK, Chang A, Darzi AW. Systems approaches to surgical quality and safety: from concept to measurement. Ann Surg 2004; 239(4):475-482.

11. Reason JT, Carthey J, de Leval MR. Diagnosing "vulnerable system syndrome": an essential prerequisite to effective risk management. Qual Health Care 2001; 10 Suppl 2:ii21-ii25.

12. Reason J. Human error: models and management. West J Med 2000; 172(6):393-396.

13. Reason J. Combating omission errors through task analysis and good reminders. Qual Saf Health Care 2002; 11(1):40-44.

14. Reason J. Beyond the organisational accident: the need for "error wisdom" on the frontline. Qual Saf Health Care 2004; 13 Suppl 2:ii28-ii33.

15. Lingard L, Espin S, Whyte $S$ et al. Communication failures in the operating room: an observational classification of recurrent types and effects. Qual Saf Health Care 2004; 13(5):330-334.

16. Reason J. Beyond the organisational accident: the need for "error wisdom" on the frontline. Qual Saf Health Care 2004; 13 Suppl 2:ii28-ii33.

17. Reason J. Safety in the operating theatre - Part 2: human error and organisational failure. Qual Saf Health Care 2005; 14(1):56-60.

18. Espin S, Lingard L, Baker GR, Regehr G. Persistence of unsafe practice in everyday work: an exploration of organizational and psychological factors constraining safety in the operating room. Qual Saf Health Care 2006; 15(3):165-170.

19. Lingard L, Espin S, Whyte $\mathrm{S}$ et al. Communication failures in the operating room: an observational classification of recurrent types and effects. Qual Saf Health Care 2004; 13(5):330-334

20. Moss E, Maxfield D. Crucial Conversations for Healthcare: Speak Up and Help Others Do the Same, Part III. Prof Case Manag 2007; 12(3):178-180.

21. Moss E, Maxfield D. Crucial Conversations for Healthcare: How to Discuss Lack of Support, Poor Teamwork, and Disrespect, Part II of III. Prof Case Manag 2007; 12(2):112-115.

22. Moss E, Maxfield D. Silence Kills: A Case Manger's Guide to Communication Breakdowns in Healthcare Part I of III. Prof Case Manag 2007; 12(1):52-54.

23. Joice P, Hanna GB, Cuschieri A. Ergonomic evaluation of laparoscopic bowel suturing. Am J Surg 1998; 176(4):373-378.

24. Joice P, Hanna GB, Cuschieri A. Errors enacted during endoscopic surgery--a human reliability analysis. Appl Ergon 1998; 29(6):409-414.

25. McKeon LM, Oswaks JD, Cunningham PD. Safeguarding patients: complexity science, high reliability organizations, and implications for team training in healthcare. Clin Nurse Spec 2006; 20(6):298-304.

26. Spellman JR. Laparoscopic equipment troubleshooting. Todays OR Nurse 1995; 17(1):13-22. 
27. Verdaasdonk EG, Stassen LP, Hoffmann WF, van der Elst M, Dankelman J. Can a structured checklist prevent problems with laparoscopic equipment? Surg Endosc. 2008 Oct;22(10):2238-43.

28. Haynes AB, Weiser TG, Berry WR, Lipsitz SR, Breizat AH, Dellinger EP, Herbosa T, Joseph S, Kibatala PL, Lapitan MC, Merry AF, Moorthy K, Reznick RK, Taylor B, Gawande AA. A surgical safety checklist to reduce morbidity and mortality in a global population. N Engl J Med. 2009 Jan 29;360(5):491-9.

29. Vries EN de, Hollmann MW, Smorenburg SM, Gouma DJ, Boermeester MA, Development and validation of the SURgical PAtient Safety System (SURPASS) checklist. Qual Saf Health Care. 2009 Apr;18(2):121-6.

30. Nilsson L, Lindberget O, Gupta A, Vegfors M. Implementing a pre-operative checklist to increase patient safety: a 1-year follow-up of personnel attitudes. Acta Anaesthesiol Scand. 2010 Feb;54(2):176-82.

31. El Shallaly G, Cuschieri A. Optimum view distance for laparoscopic surgery. Surg Endosc. 2006 Dec;20(12):1879-82.

32. Kenge $P$, Karlsson A Human error driving the development of a checklist for foreign material exclusion in the nuclear industry: Research Articles Human Factors in Ergonomics \& Manufacturing, 2007 May, Vol 17 (3) P: $283-298$

33. Arora, B, Hull L, Sevdalis N, Tierney T, Nestel D, Woloshynowych, Darzi A, Kneebone R. Factors compromising safety in surgery: stressfull events in the operating room. Am J Surg 2010 Jan;199(1):60-5 



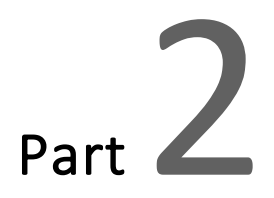

Ergonomics in minimal invasive surgery 

Towards the ideal starting posture for the surgeon during endoscopic surgery: analysis of the optimal ergonomic laparoscopic position in a simulation environment

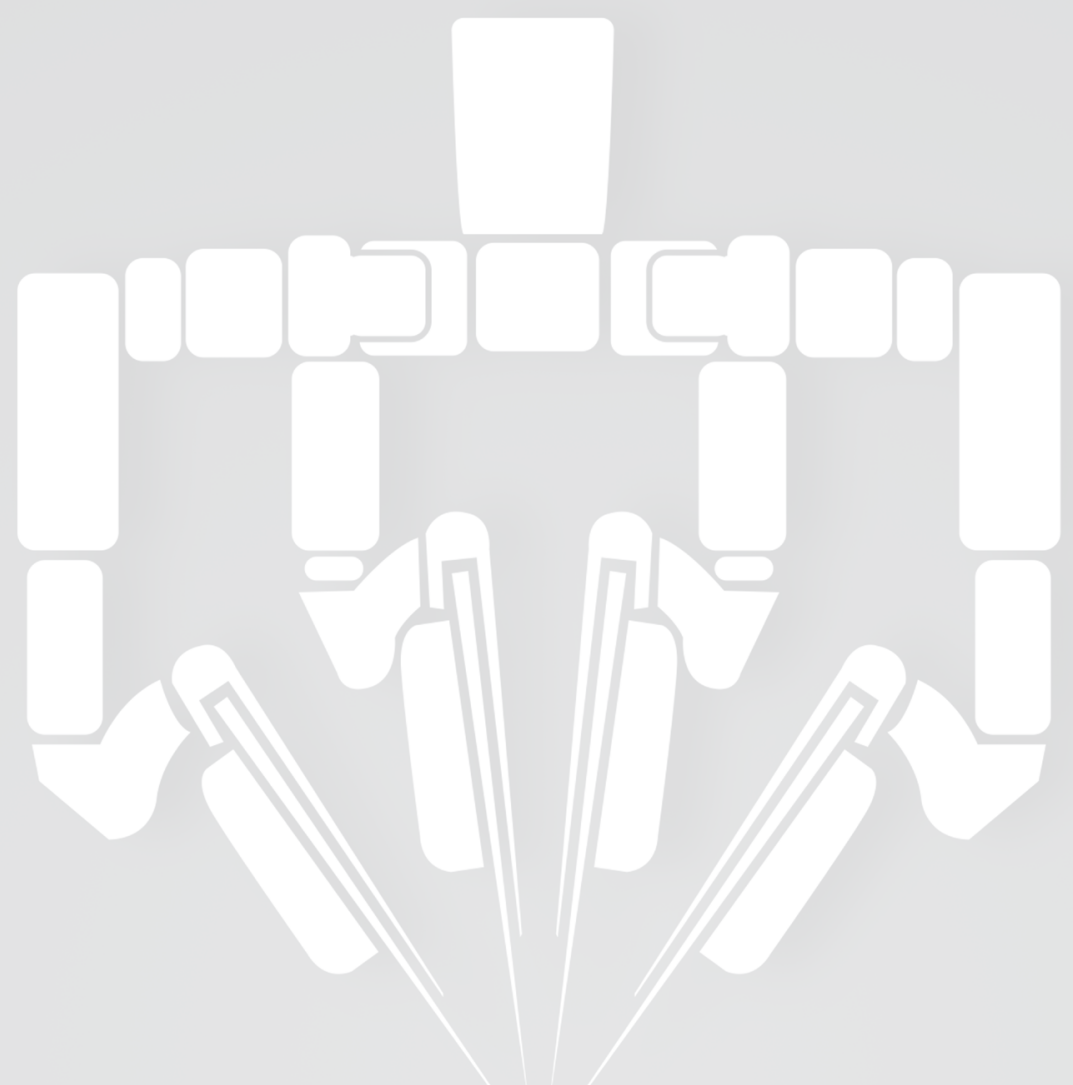

C. van 't Hullenaar, M. van Alphen, M. Hendriks, I. Broeders Research project performed at Laboratory of Biomechanical Engineering, 
Chapter 4 


\section{Background}

Over a hundred years ago, Professor Fritz de Quervain from Switzerland demonstrated the importance of operation table height and the position of the patient in relation to the surgeon. His goal was to improve ergonomics during open surgical procedures [18]. Since then, many aspects of the operating room environment have changed. Some of these included the introduction of better light sources and adjustable tables. Also, new technologies and instruments with an improved ergonomic design were developed. The introduction of endoscopic surgery in the 1980s generated various documented benefits to patients [14]. However, an endoscopic procedure is a technical task that is physically demanding on the surgeon. Trocars influence his range and way of motion. Instruments are at work indirectly at the point of interest. Visualization becomes a multifactor process. These factors can confront endoscopic surgeons with compromised ergonomics. Uncomfortable postures can cause excessive fatigue and may lead to physical discomfort after surgery $[8,13]$. Improvement of ergonomics is essential to reducing fatigue, discomfort and physical damage.

Ergonomics during endoscopic surgery have been evaluated in previous studies [1, 3, 4 and 12]. Berguer et al. demonstrated a reduction of posture changes during endoscopic surgery. This causes fatigue during those interventions [3]. In 1999, Berguer et al. determined that $8 \%$ to $12 \%$ of endoscopic surgeons experienced frequent pain in the neck and upper extremities after an endoscopic procedure [1]. Berguer et al. reported that performing complex endoscopic tasks leads to high upper extremity muscle tension. Improvement of ergonomics is not only important for the surgeon; it can potentially also affect patient outcomes. Several studies investigated key ergonomic aspects with regard to endoscopic operations. Table height [10], monitor placement [7,11,12, and 15], design of instruments [2,9] and the use of foot pedals [8] were independent variables that strongly correlate with changes to the ergonomic situation.

Ergonomic studies determined that many tasks are best performed when the surgeon is positioned in an ideal and comfortable position. Determining the ideal position for performing endoscopic surgery is essential in studies with a focus on ergonomics in the operating room. This study sees a new method developed to determine the ideal starting position for performing endoscopic surgery. The goal of this study is to find the ideal starting posture for surgeons during endoscopic surgery in an experimental laboratory setting.

\section{Materials and methods}

At the University of Twente, twenty-two students with no experience in endoscopic surgery volunteered to participate in our project. This experimental setting saw any operating room obstacles removed. An optical detection system (VICON) was used in the Laboratory of Biomechanical Engineering to determine the three-dimensional positions of the reflective markers that were affixed onto the subjects. Eight infrared cameras were 
installed to detect these reflective markers. The markers were affixed in such a way that angles of different joints and the rotation of the torso could be calculated.

Two markers were affixed to the posterior and anterior side of the acromion and another to the lateral epicondyle of the humerus. Markers were affixed to the medial and lateral side of the wrist and at the base of the middle finger so as to determine the position of the arms. Three markers were affixed to the subject's head (one anterior, one posterior, left, and one posterior, right). Finally, three markers were affixed to the instruments, where one marker was affixed to the connected tips and one to the midpoint of each instrument (see Figure 1). All subjects were requested to stand in their most comfortable position, holding two endoscopic instruments that were connected at their tips. This simulates a working situation where a surgeon would be dissecting using two instruments. Calibration of the VICON system was performed prior to each measurement. Following calibration, the data regarding the upper extremity position were gathered. This method allowed the optimal comfortable starting postures to be recorded. Four measurements were taken for each subject. The three-dimensional VICON coordinates were automatically loaded onto a computer. Transcription of these data was done using associated software. Calculations of different angles in joints were done in Matlab R2010a ${ }^{\circledR}$ using specifically designed scripts.

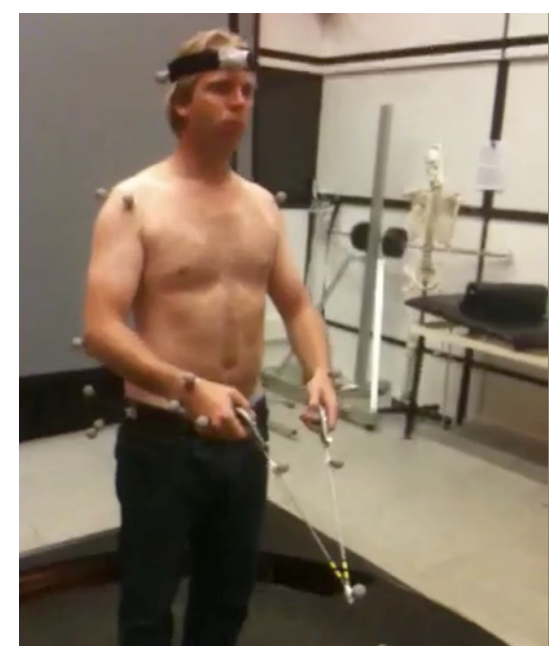

Figure 1: Position of the markers

The joint angles that were calculated using the VICON system are displayed in Figure 2 and are further explained in Table 1. The angles were calculated by defining two lines between the markers of interest. For instance, the line from the acromion to the lateral epicondyle and the line from the lateral epicondyle to the middle of the hand were taken to calculate the angle of the elbow. These two lines allowed us to determine the angle. Triangular surfaces were created to calculate the angles of the head. Those triangular 
surfaces allowed us to conduct an accurate analysis of those angles. Once the angle of the three head markers had been calculated using a horizontal surface, flexion and extension of the neck could also be determined. A positive angle displays head extension; a negative angle is associated with flexion of the cervical spine. These also enabled us to calculate the abduction and anteflexion of the arms and the rotation of the trunk. Mean values in all angles were computed. We included the three most consistent records in our analysis.

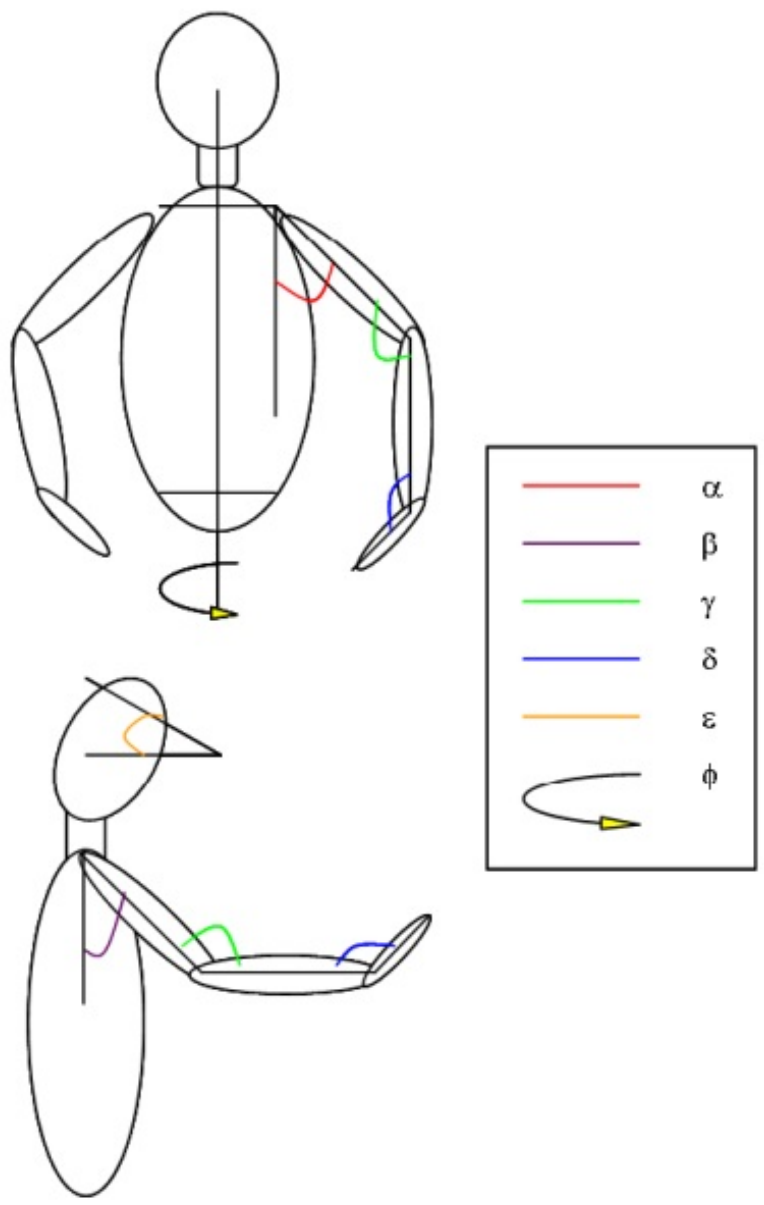

Figure 2: The angles measured by using the VICON system. 


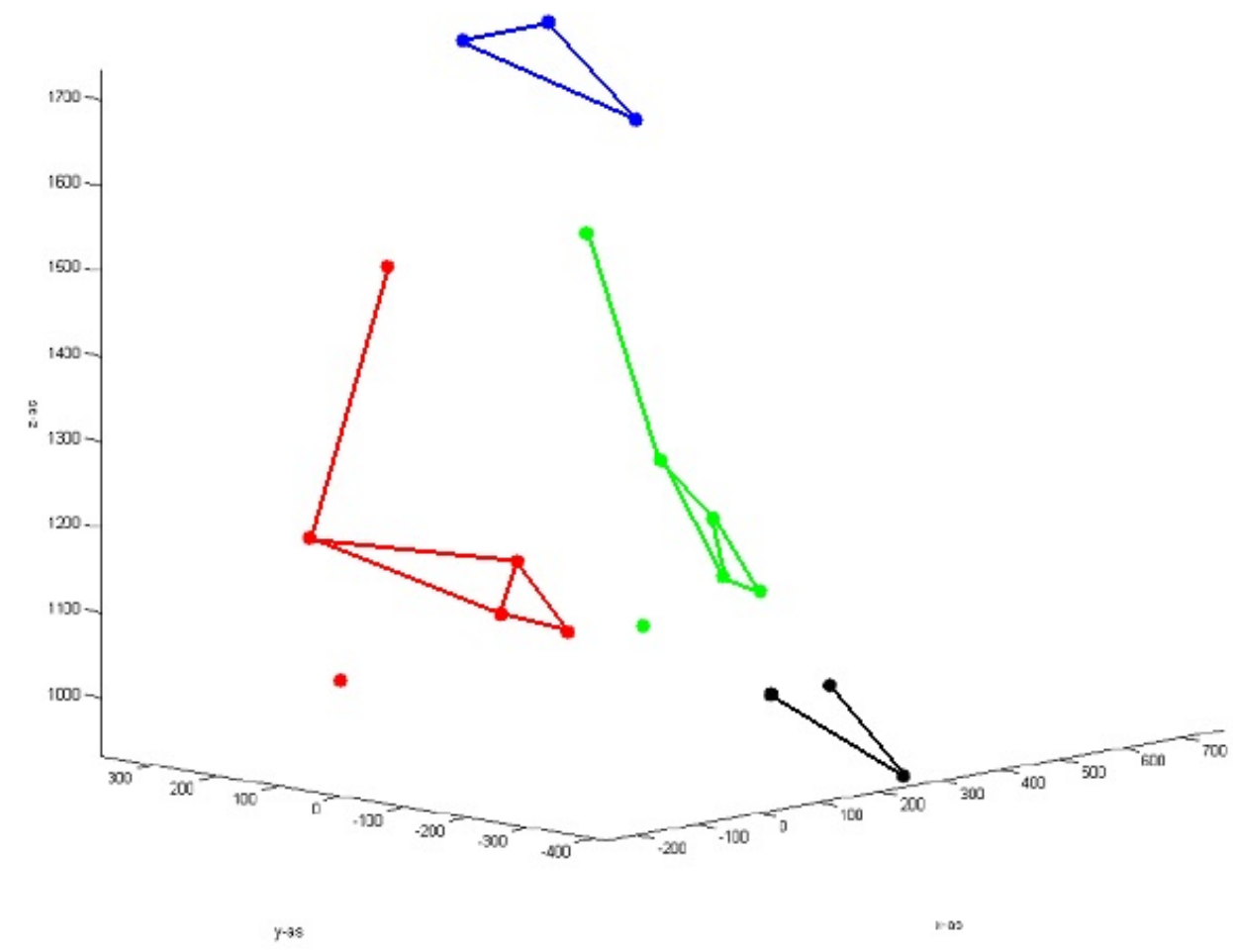

Figure 3: Representation of plotted data to determine the three-dimensional positions of the reflective markers.

\section{Results}

The main results can be found displayed in Table 2. Average values for abduction were found to be at $19^{\circ}$ for the left arm, and at $18^{\circ}$ for the right side, respectively. The average position of the left arm was at $15^{\circ}$ anteflexion, for the right arm this angle was at $12^{\circ}$. A symmetric position of the elbows was recorded; the angle of both elbows (flexion/extension) was determined at $94^{\circ}$. Minimal rotation of the torso was computed and an average rotation of $5^{\circ}$ was found. The calculated average angle between the two instruments was determined at $28^{\circ}$. The view-instrument-axis was therefore determined at $14^{\circ}$ (half the instrument-axis). Average cervical spine angle was determined at $11^{\circ}$ of neck flexion. Table 2 lists the both the overall average and standard deviations, as well as those per subject. Figure 3 includes an example of one of the measurements. 
Table 1: Description of all calculated angles

\begin{tabular}{ll}
\hline Angle & Description \\
\hline$\alpha$ & Abduction of the shoulder \\
$\beta$ left & Anteflexion of the shoulder \\
$\gamma$ left & Flexion of the elbow \\
$\varepsilon$ & Extension of the cervical spine (negative value = flexion) \\
$\phi$ & Rotation of the spine \\
$\eta$ & Instrument angle \\
\hline
\end{tabular}

Table 2: Average angles (in degrees) and the standard deviation of the measurements per subject for the defined angles. The asterisk $(*)$ represents missing data.

\begin{tabular}{|c|c|c|c|c|c|c|c|c|c|}
\hline Subject & $\alpha$ left & $\alpha$ right & $\beta$ left & $\beta$ right & $\gamma$ left & v right & $\varepsilon$ & $\phi$ & $\eta$ \\
\hline 1 & $20(0.2)$ & $(11.0)$ & $(5.0)$ & $15(1.0)$ & $105(6.0)$ & $104(3.0)$ & $-20(6.6)$ & $5(0.2)$ & $31(1.2)$ \\
\hline 2 & $16(0.2)$ & $18(0.3)$ & $11(0.0)$ & $10(0.5)$ & $92(0.3)$ & $92(0.5)$ & $-3(4.6)$ & $1(0.1)$ & $21(0.3)$ \\
\hline 3 & * & $*$ & * & $*$ & $79(2.0)$ & $91(1.0)$ & $0.0(1.4)$ & $*$ & $30(2.0)$ \\
\hline 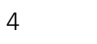 & $14(0.2)$ & $15(2.0)$ & $16(1.0)$ & $18(0.1)$ & 73 (12.0) & $80(3.0)$ & $-10(5.0)$ & $6(0.5)$ & 30 (1.9) \\
\hline 5 & $13(3.0)$ & $13(1.0)$ & $9(7.0)$ & $3(0.0)$ & $91(7.6)$ & $84(3.5)$ & $+4(0.3)$ & $4(0.0)$ & $20(5.0)$ \\
\hline 6 & $19(0.4)$ & $19(1.0)$ & $(0.0)$ & $6(1.0)$ & $107(12.0)$ & $106(5.0)$ & $-3(4.7)$ & $4(0.0)$ & $24(1.0)$ \\
\hline 7 & $13(0.2)$ & $13(1.0)$ & $10(3.0)$ & $4(2.0)$ & $98(4.0)$ & $100(4.0)$ & $-14(4.0)$ & $5(0.0)$ & $33(2.0)$ \\
\hline 0 & $13(1.0)$ & $11(3.0)$ & $13(9.0)$ & $5(3.0)$ & $95(5.0)$ & $92(4.0)$ & $-14(0.2)$ & $8(0.9)$ & $30(5.0)$ \\
\hline 0 & $47(0.0)$ & $24(4.0)$ & $21(0.0)$ & $5(1.0)$ & $89(13.0)$ & $91(2.0)$ & $-15(0.9)$ & $3(0.5)$ & $42(6.0)$ \\
\hline 10 & $13(5.0)$ & $14(4.0)$ & $11(3.0)$ & $7(0.2)$ & $142(4.8)$ & $140(2.8)$ & $-4(4.0)$ & $3(0.5)$ & $28(0.0)$ \\
\hline 11 & $13(4.0)$ & $15(0.0)$ & $8(0.0)$ & $12(1.0)$ & $93(3.3)$ & $87(1.2)$ & $-9(1.0)$ & $4(0.5)$ & $23(0.5)$ \\
\hline 12 & $25(0.3)$ & $20(0.3)$ & $24(1.0)$ & $19(0.1)$ & $78(2.0)$ & $77(1.0)$ & $-9(0.1)$ & $5(0.0)$ & $23(1.0)$ \\
\hline 13 & $35(0.0)$ & $17(0.3)$ & $23(0.0)$ & $8(0.2)$ & $115(3.0)$ & $124(4.8)$ & $-12(2.0)$ & $6(1.4)$ & $18(2.0)$ \\
\hline 14 & $23(12.0)$ & $25(13.0)$ & $19(6.0)$ & 21 (11.0) & $89(6.0)$ & $89(3.5)$ & $+2(0.8)$ & $6(0.0)$ & $29(2.0)$ \\
\hline 15 & $16(0.1)$ & $14(0.0)$ & $15(0.1)$ & $14(0.3)$ & $95(0.0)$ & 91 (1.0) & $-11(2.0)$ & $3(0.1)$ & $24(0.1)$ \\
\hline 16 & $26(0.0)$ & $23(0.1)$ & $21(0.0)$ & $12(0.2)$ & $81(0.0)$ & $87(0.1)$ & $-9(0.1)$ & $3(0.1)$ & $21(0.3)$ \\
\hline 17 & $20(0.4)$ & $15(1.0)$ & $9(3.0)$ & $10(0.3)$ & $113(5.0)$ & $108(2.0)$ & $-12(0.7)$ & $8(0.2)$ & $39(2.0)$ \\
\hline 18 & $19(1)$ & $23(1.0)$ & $7(0.1)$ & $7(0.5)$ & $116(14.0)$ & $111(5.0)$ & $-42(4.0)$ & $8(0.7)$ & $32(1.0)$ \\
\hline 19 & $6(1.0)$ & $14(1.0)$ & $10(2.0)$ & $18(1.0)$ & $74(9.0)$ & 75 (1.0) & $-20(1.0)$ & $6(0.0)$ & $50(1.0)$ \\
\hline 20 & $19(1.0)$ & $18(3.0)$ & $20(1.0)$ & $15(1.0)$ & $88(1.0)$ & $94(3.0)$ & $-22(1.9)$ & $2(1.0)$ & $23(1.4)$ \\
\hline 21 & $10(0.1)$ & $18(0.2)$ & $24(0.1)$ & $31(0.2)$ & $74(0.3)$ & $71(1.0)$ & $-8(0.3)$ & $8(0.0)$ & $26(1.0)$ \\
\hline 22 & $16(1.0)$ & $17(0.1)$ & $22(2.0)$ & $12(1.0)$ & $75(3.0)$ & $80(1.0)$ & $-18(0.6)$ & $8(0.0)$ & $28(1.0)$ \\
\hline Mean & 19 & 18 & 15 & 12 & 94 & 94 & -11 & 5 & 28 \\
\hline
\end{tabular}

\section{Discussion}

Despite the fact that many endoscopic surgeons suffer from physical discomfort during surgery, only limited data are available on the ergonomics in play during these procedures. The studies on ergonomics in endoscopic surgery published to date, have determined optimal table heights, instrument angles and monitor positions. Several 
papers quantified ergonomics by applying a Visual Analogue Scale to detect uncomfortable positions $[3,16]$. Neither of these studies focused on the ideal joint angles in a situation where operating room obstacles had been removed. This study is based on an approach where the ideal starting position is not limited by factors such as table height and monitor distance. By determining the ideal starting position in a simulation environment, one becomes able to better understand compromised ergonomics during endoscopic procedures.

The data presented in this paper are consistent with the results published in previous studies. Berguer et al. [2] found an optimal angle between the work axis and the view axis of less than $15^{\circ}$. This was confirmed by electromyography recordings. In our research project, the VICON system recorded an average angle between the instruments of $28^{\circ}$. That means a view-instrument-axis of $14^{\circ}$, this is also analogous with previous data. Van Det et al. [6] describe in their review an optimal cervical spine flexion of $15^{\circ}$; this is consistent with our data. Regarding trunk rotation, having no rotation in the lumbar and thoracic spine is considered optimal. As expected, our data are in accordance with that assertion.

Several limitations of this study need to be addressed, however. Twenty-two students with no experience in endoscopic surgery volunteered to participate in this project. One could state that this group is ignorant to the potential obstacles present in the operating room. The advantage to this is that the students were able to demonstrate an unbiased ergonomic position. On the other hand, taking up a certain position for a short period of time in a laboratory setting, is different to performing an actual surgical procedure in an operating theatre. That is why these results cannot be directly extrapolated to an operating room environment.

Measurements were taken using the VICON infrared system. This system is based on technology that features exact locations of anatomical landmarks represented by reflective markers. All markers were manually positioned on predetermined anatomical landmarks. This can potentially incur a certain level of inaccuracy. Failures in marker placement can lead to the recording of inaccurate angles. However, given that most recordings showed a symmetric angle on the left as well as the right side, this effect appears insignificant.

Endoscopic surgery is a dynamic process where continuous changes in movement may occur. In our laboratory set-up, two instruments were connected at their tips in an attempt to mimic an actual target area. We reproduced the reality of a starting position in the operating room. The data of this study are vital to gaining a better understanding of the ideal position in endoscopic surgery. The ideal starting position we found, is reflective of an optimal ergonomic situation. Fatigue to muscles and joints is likely to set in at a slower rate when starting the procedure from this ideal position.

This study aimed to determine the optimal physical conditions at the start of a procedure. We are advocating that an optimal starting position be adopted in order to reduce physical strains during endoscopic procedures. The data generated by this study, 
can serve as a platform for further research. Further studies need to determine whether the starting posture we defined, can be applied to an actual operating room setting. Of course, many surgical components such as foot pedals, table height, screen and trocar position can influence ergonomics. It is therefore vital to obtain more knowledge about the ergonomics in the endoscopic operation room. That could, in turn, lead to improvements to hardware, instruments and team performance.

\section{Conclusions}

The ideal starting posture when performing endoscopic surgery can be defined in a laboratory setting. This ideal posture embodies a mild level of neck flexion, no trunk rotation and a moderate $15^{\circ}$ anteflexion of the upper arm. Also, a slight upper arm abduction $\left(20^{\circ}\right)$ is considered ideal. Average elbow flexion was found to reside at $96^{\circ}$. The ideal angle between the two endoscopic instruments was determined at $27^{\circ}$. Our data are in accordance with previous findings in literature and can serve as a platform for further ergonomic research. 


\section{References}

1. R Berguer, D L Forkey, and W D Smith. Ergonomic problems associated with laparoscopic surgery. Surgical Endoscopy, 13:466\{468, 1999.

2. R Berguer, D L Forkey, and W D Smith. The effect of laparoscopic instrument working angle on surgeons' upper extremity workload. Surgical Endoscopy, 15:1027\{1029, 2001.

3. R Berguer, G T Rab, A Alarcon, and J Chung. A comparison of surgeons posture during laparoscopic and open surgical procedures. Surgical Endoscopy, 11:139\{142, 1997.

4. Ramon Berguer, Jerry Chen, and Warren D Smith. A Comparison of the Physical Effort Required for Laparoscopic and Open Surgical Techniques. Archives Surgery, 138:967\{970, 2003.

5. Sara L Best and Jeffrey A Cadeddu. Use of Magnetic Anchoring and Guidance Systems to Facilitate Single Trocar Laparoscopy. Current Urology Reports, 11:29\{32, 2010.

6. M J Van Det, W J H J Meijerink, C Hof, E R Totte, and J P E N Pierie. Optimal ergonomics for laparoscopic surgery in minimally invasive surgery suites : a review and guidelines. Surgical Endoscopy, 23:1279\{1285, 2009.

7. George B Hanna, Sami M Shimi, and Alfred Cuschieri. Task Performance in Endoscopic Surgery IS Influenced by Location of the Image Display. Annals of Surgery, 227(4):481\{484, 1998.

8. Georgios Haramis, Juan Carlos Rosales, Jorge Moreno Palacios, Zhamshid Okhunov, Adam C. Mues, Diana Lee, Ketan Badani, Mantu Gupta, and Jaime Landman. Prospective Randomized Evaluation of FOOT Gel Pads for Operating Room Staff Comfort During Laparoscopic Renal Surgery. in press, 2010.

9. U Matern, G Kuttler, C Giebmeyer, P Waller, and M Faist. Ergonomic aspects of five different types of laparoscopic instrument handles under dynamic conditions with respect to specific laparoscopic tasks: An electromyographic-based study. Surgical Endoscopy,18:1231\{1241, 2004.

10. U Matern, Peter Waller, Carsten Giebmeyer, Klaus D Ruckauer, and Eduard H Farthmann. Ergonomics: Requirements for Adjusting the Height of Laparoscopic Operating Tables. Journal of the Society of Laparoendoscopic Surgeons, 5:7\{12, 2001.

11. A M Omar, N J Wade, S I Brown, and A Cuschieri. Assessing the benefits of gaze-down display location in complex tasks. Surgical Endoscopy, 19:105\{108, 2005.

12. Adrian Park, Gyusung Lee, Jacob Seagull, Nora Meenaghan, and David Dexter. Patients Benefit While Surgeons Suffer: An Impending Epidemic. American College of Surgeons, 210(3):306\{311, 2010.

13. R H Van Der Schatte Olivier, C D P Van 't Hullenaar, J P Ruurda, and I A M J Broeders. Ergonomics, user comfort, and performance in standard and robot-assisted laparoscopic surgery. Surgical Endoscopy, 23:1365\{1371, 2009.

14. Abdul Razak Shaikh, Ahmed Khan Sangrasi, and Gulshan Ara Shaikh. Clinical Outcomes of Laparoscopic Versus Open Appendectomy. Journal of the Society of Laparoendoscopic Surgeons, 13:574 \{580, 2009.

15. M A Van Veelen, J J Jakimowicz, R H M Goossens, D W Meijer, and J B J Bussmann. Evaluation of the usability of two types of image display systems, during laparoscopy. Surgical Endoscopy, 16:674\{678, 2002.

16. M A Van Veelen, E A L Nederlof, R H M Goossens, C J Schot, and J J Jakimowicz. Ergonomic problems encountered by the medical team related to products used for minimally invasive surgery. Surgical Endoscopy, 17:1077 1081, 2003.

17. Cavallo, Filippo PhD; Pietrabissa, Andrea MD; Megali, Giuseppe PhD; Troia, Elena PhD; Sinigaglia, Stefano PhD; Dario, Paolo MD; Mosca, Franco MD; Cuschieri, Alfred MD. Proficiency Assessment of Gesture Analysis in Laparoscopy by Means of the Surgeon's Musculo-Skeleton Model Annals of Surgery . 255(2):394-398, February 2012.

18. De Quervain F. Weiteres zur Operationstischfrage. Zentralbl Chir. 1909;19:686-688. 


\section{Chapter}

\section{Ergonomics, user comfort and performance in standard and robot-assisted laparoscopic surgery}

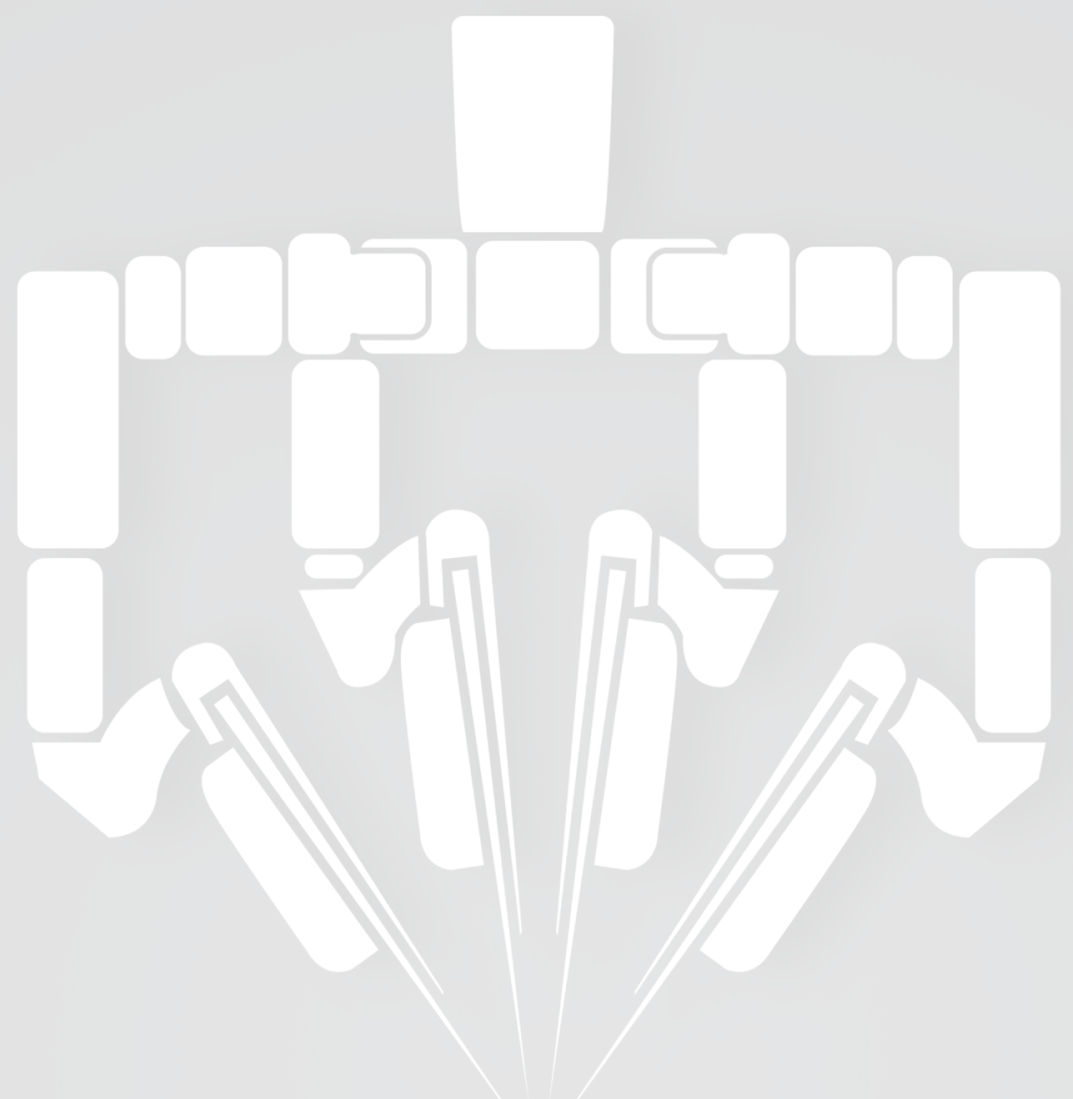

R. van der Schatte Olivier, C. van 't Hullenaar, J. Ruurda, I. Broeders Surgical Endoscopy (2009) Jun; 23(6):1365-71. 


\begin{abstract}
Background Robot-assisted surgical systems have been introduced to improve the outcome of minimally invasive surgery. These systems also have the potential to improve ergonomics for the surgeon during endoscopic surgery. This study aimed to compare the user's mental and physical comfort in performing standard laparoscopic and robotassisted techniques. Surgical performance also was analysed.

Methods In this study, 16 surgically inexperienced participants performed three tasks using both a robotic system and standard laparoscopic instrumentation. Distress was measured using questionnaires and an ambulatory monitoring system. Surgical performance was analysed with time-action analysis.
\end{abstract}

Results The physiologic parameters $(p=0.000)$, the questionnaires $(p=0.000)$, and the time-action analysis ( $p=0.001$ ) favoured the robot-assisted group in terms of lower stress load and an increase in work efficiency.

Conclusion In this experimental setup, the use of a robot- assisted surgical system was of value in both cognitive and physical stress reduction. Robotic assistance also demonstrated improvement in performance.

Keywords Ergonomics, Laparoscopy, Minimally invasive surgery, Robotics, Stress

\title{
Abbreviations
}

AMS Ambulatory monitoring system

HRA Heart rate average

MSSD Mean square of successive differences between consecutive heartbeats

PEP Pre-ejection period

RA Robot assisted

RSA Respiratory sinus arrhythmia

SL Standard laparoscopic 
Laparoscopic surgery offers distinct advantages to patients over open surgery such as shorter hospitalization, reduced postoperative pain, and better cosmetic results [21, 29, 31]. On the other hand, laparoscopic surgery is technically and physically demanding for the surgeon due to limitations in manipulation and visualization as well as physical discomfort $[2,4,5,10,14,16,23-25]$.

Robot-assisted surgical systems were developed to overcome many of the surgical disadvantages associated with laparoscopic surgery. Manipulation of the instruments is improved by increased range of motion for the instruments, correction of the inverted instrument response, and elimination of human tremors [1, 8, 20, 27]. Furthermore, vision of the operative field is improved by conversion of a two dimensional (2D) image to a 3D view. Additional advantages are a completely stable image and personal camera control. The physical discomfort of laparoscopic instrument handling is potentially reduced by restoration of the eye-hand-target working axis and physical support of the console $[3,18,24,26]$. These advantages may reduce both the physical and mental workload, thus possibly improving both surgical performance and work satisfaction. This experiment aimed to assess whether robot-assisted surgery benefits the surgeon by reducing mental and physical discomfort in comparison with standard laparoscopy. Additionally, task performance was evaluated to assess whether robot assistance can benefit surgical procedures [22].

\section{Methods}

In this study, 16 medical students ( 8 women and 8 men) each performed three tasks. These tasks were executed in a pelvic trainer in random order using both standard laparoscopic instruments (standard group) and the da Vinci robot-assisted surgical system (Intuitive Surgical, Sunnyvale, CA, USA) (robot-assisted group).

The participants were instructed in the tasks and techniques. To become familiar with the setup of the exercise, both the standard group and the robot-assisted group were allowed to practice each task for $2.5 \mathrm{~min}$. All the tasks were executed for $5 \mathrm{~min}$ with the objective to achieve as many repetitions as possible. The participants had a 15-min break after finishing all three tasks, during which they were asked to complete the questionnaires. After the break, the participant performed all three tasks again, but now in the other group. The tasks were rope passing, needle capping and bead dropping. All tasks are described here below. At the end of this second session, the participants completed the questionnaires again. To encourage maximal performance, the most accomplished participant was given a reward.

In the standard laparoscopic group, table height was adjusted to the individual's preference. A horizontal instrument angulation less than $45^{\circ}$ and a vertical angulation less than $15^{\circ}$ [4] were always preserved. The monitor trolley was placed in line with the camera at a distance of $1.5 \mathrm{~m}$ from the pelvic trainer. The participants performed all the tasks using two dissection forcipes (B. Braun, Tuttlingen, Germany). 
The robot-assisted group worked at the console, from which they controlled a threearmed da Vinci robot. Before onset of the tasks, each participant adjusted the console to his or her personal preference with regard to height of the chair, display clarity, optimal stereoscopic view, and comfortable armrest position. Both groups performed the same three tasks, and both groups performed all tasks with the same triangulation in the port placement setup.

\section{Surgical tasks}

The following surgical tasks were performed.

Rope passing

A 25 -cm-long rope (diameter $0.3 \mathrm{~cm}$ ) was passed as many times as possible. The rope was grasped alternately with the left and right instruments at the 11 marked grasping points. During this exercise, the participants were instructed to keep the rope above the floor of the training box.

Needle capping

A needle and a cap were grasped and lifted, after which the needle was capped repeatedly above the floor of the training box.

Bead dropping

Every participant was asked to pick up a plastic bead from a starting position, transfer it crosswise, and drop it into a receptacle. After the bead was dropped into the receptacle, it had to be picked up and transferred to another receptacle using the contralateral laparoscopic instrument.

\section{Physiologic measurements of mental discomfort}

An ambulatory monitoring system was used for registration of physical parameters. It could be worn beneath clothing, allowing the subject complete freedom of movement $[12,28,32]$. Three physiologic stress parameters were recorded with this device. The first parameter was the mean square of successive differences between consecutive heartbeats (MSSD). This parameter reflects the beat-to-beat variability of the heart rate and is tightly linked to respiratory sinus arrhythmia (RSA). Changes in RSA can display changes in vagal activity [6]. If vagal activity decreases RSA, MSSD also will be reduced because fewer oscillatory changes in heart rate occur. An increase in stress will therefore lead to a decrease in MSSD. In conclusion, high MSSD values reflect low stress levels.

The second parameter recorded was the preejection period (PEP: time of isovolumetric contraction). The PEP is the interval between the onset of ventricular depolarization and the opening of the semilunar valves [19]. Changes in PEP correspond to changes in $ß$-adrenergic inotropic drive to the left ventricle. This correlation was shown by laboratory studies in which ß-adrenergic tone was manipulated by epinephrine infusion [15, 30], adrenoceptor blockade [13], exercise [14, 17], and emotional stress [7, 18]. 
The third parameter was the average heart rate (HRA) of the participants recorded by the ambulatory monitoring system.

All three parameters (MSSD, PEP, HRA) were constantly recorded during both the laparoscopic tasks and the robot-assisted tasks. The data were automatically divided by the ambulatory monitoring system device into 30 second periods. The average of these 30 second recordings was compared with a paired sample $t$-test using SPSS software, version 13.0 for Windows (SPSS Inc., Chicago, IL, USA).

\section{Questionnaires}

The participants completed two questionnaires. To score the level of stress, all the participants completed the Subjective Mental Effort Questionnaire (SMEQ) and the Local Experienced Discomfort (LED) scale. The SMEQ is a cognitive workload questionnaire with a scale of 0 to 150 points (Appendix 1). It is designed so that individuals can rate the amount of effort invested during a task. Nine scale markers with verbal statements ranging from "no effort at all" to "exceptional amount of effort" are displayed in the SMEQ diagram. The choice of statements and their scale locations are empirically derived. The SMEQ, used as a tool in various laboratory and field studies, is categorized as a cognitive test [33].

The LED allowed participants to express their physical discomfort during performance of all the tasks [9]. On a scale of 0 to 10 points, the participants were asked to identify their physical discomfort at several locations of the upper body (Appendix 2). Statistical analysis of both questionnaires was performed using a Mann-Whitney $U$ test.

\section{Task performance}

All the procedures were recorded with a super-VHS recorder. Using these recordings, failures and successes of executed repetitions were applied to compare task performance between the two groups. The judgment for success or failure of a given task was based on consensus of the researchers.

\section{Results}

\section{Physiologic measurements}

Table 1 presents the physiologic measurements. The mean MSSD in the robot-assisted group was $31.7 \mathrm{~ms}$. This was significantly higher than in the standard laparoscopic group, which had a value of $22.3 \mathrm{~ms}(t=-4.0 ; p=0.01)$. The results of the PEP also were in favour of the robot- assisted group. A mean PEP value of $131.6 \mathrm{~ms}$ was recorded in the robotassisted group, compared with a mean PEP value of $126.0 \mathrm{~ms}$ in the standard group $(t=$ 3.3; $p=0.004)$.

The average HRA was significantly higher in the standard laparoscopy group. A value of 90.5 beats/min was recorded in the standard laparoscopy group compared with a value of 79.9 beats $/ \mathrm{min}(t=5.1 ; p=0.0001)$ calculated for the robot-assisted group. 


\section{Questionnaires}

The SMEQ questionnaire showed a median physical effort in the robot-assisted group of 30 points (range, 10-70), whereas a median of 80 points (range, 50-100) was reported for the standard laparoscopic group $(p=0.001)$. The LED score for the physical discomfort experienced was 7.4 points (range, $0-25$ ) in the standard laparoscopic group compared with 1.3 points (range, $0-9$ ) in the robot- assisted group ( $p=0.001$ ). The median number of physical complaints was 1 (range, $0-8$ ) in the standard group and 0 (range, $0-8$ ) in the robot-assisted group ( $p=0.003)$.

\section{Time-action analysis}

In each of the executed tasks of the experiment, the participants performed significantly more repetitions with robot assistance (Table 2). Additionally, fewer failures in task performance were recorded in the robot-assisted group than in the laparoscopic group. This discrepancy was statistically significant $(p<0.001)$.

Table 1 Physiologic measurements of the ambulatory monitoring system ${ }^{\mathrm{a}}$

\begin{tabular}{lllll}
\hline & Standard laparoscopy group & robot-assisted group & $t$-value & $p$-value \\
\hline MSSD (ms) & $22.3(8.0-73.4)$ & $31.7(15.0-53.4)$ & -4.0 & 0.01 \\
PEP (ms) & $126.0(106.5-142.4)$ & $131.6(119.0-146.2)$ & 3.3 & 0.004 \\
HRA (beats/min) & $90.5(73.8-109.4)$ & $79.9(66.9-98.0)$ & 5.1 & 0.0001 \\
\hline
\end{tabular}

MSSD mean square of successive differences between consecutive heartbeats, PEP between consecutive heartbeats, HRA average heart rate

${ }^{a}$ Data are presented as mean value (range)

Table 2 Results of the action analysis ${ }^{\mathrm{a}}$

\begin{tabular}{|c|c|c|c|c|c|}
\hline & \multicolumn{2}{|c|}{ Standard lap } & \multicolumn{2}{|c|}{ robot-assisted } & \multirow[t]{2}{*}{$p$-value } \\
\hline & Median & Range & Median & Range & \\
\hline Rope passing & 3.5 & $1.0-7.5$ & 8.3 & $5.5-14.0$ & 0.000 \\
\hline Failure to pass the rope & 5.0 & $1.0-4.0$ & 1.5 & $0.0-5.0$ & 0.000 \\
\hline Needle capping & 1.0 & $0.0-6.0$ & 5.0 & $2.0-10.0$ & 0.000 \\
\hline \multicolumn{6}{|l|}{ Failure to cap the needle } \\
\hline Missing the cap & 13.0 & $2.0-25.0$ & 0.5 & $0.0-9.0$ & 0.000 \\
\hline Dropping the needle & 6.5 & $4.0-14.0$ & 2.0 & $1.0-9.0$ & 0.001 \\
\hline Bead drop & 22.0 & $3.0-45.0$ & 48.0 & $28.0-69.0$ & 0.000 \\
\hline $\begin{array}{l}\text { Failure to drop the bead into the } \\
\text { receptacle }\end{array}$ & 3.0 & $1.0-7.0$ & 0.0 & $0.0-5.0$ & 0.001 \\
\hline
\end{tabular}

\footnotetext{
${ }^{a}$ Number of repetitions is expressed as median and range. Mann-Whitney $U$ test used to calculate $p$ value
} 


\section{Discussion}

This study aimed primarily to assess whether robot-assisted surgery can benefit the surgeon in terms of stress reduction and physical comfort. In general, stress can be defined as a specific response by the body to a stimulus that disturbs or interferes with the normal physiologic equilibrium. This physical, mental, or emotional tension of an individual decreases the feeling of being in control of a given situation.

Surgeons control the surgical environment usually by means of mental and physical skills, in cooperation with operating room colleagues and the available equipment. Improving either one of these aspects can increase situational control. In this experiment, the focus was placed on the equipment.

Robot-assisted surgical systems are designed to facilitate manipulation of surgical instruments by increasing freedom of movement and introducing stereoscopic vision. Additionally, physical workload is reduced as the surgeon operates from a comfortable console instead of standing next to the operating table [14]. Ergonomics and user comfort in robot-assisted laparoscopic surgery have not gained much scientific interest but probably are underestimated in an era when more endoscopic procedures are being performed every year, both in basic and complex surgery. A recent study suggests a possible decrease in mental stress with the implementation of robotic techniques, but statistical significance to support this finding has not been established [5].

Our study confirmed that robotic assistance can reduce physical stress significantly. The objective recordings of the ambulatory monitoring system device (MSSD, PEP, HRA) demonstrated robot-assisted surgery to be less stressful than standard laparoscopy. Besides these physical parameters, the mental questionnaires also displayed a significant decrease in mental and physical effort when laparoscopic tasks were performed with the assistance of a robot.

The questionnaires demonstrate that robotic assistance reduced the cognitive workload $(p<0.0001)$, the amount of physical discomfort $(p=0.001)$, and the total number of complaints $(p=0.003)$. The performance of all the tasks in the robotic group was significantly better, with more repetitions and fewer failures.

These results all are highly suggestive of stress reduction. However, some critical remarks must be made. First, physical and mental stress in simulated, experimental conditions is not directly comparable with stress in actual surgical situations. In actual procedures, with teamwork also playing a substantial role, the working environment can be more complex and demanding. The seated position of the participants in the robotassisted group must be taken into account as well.

Second, with a recording time of 15 min (excluding 7.5 min of practice time), the "operating time" was relatively short. It is likely that more physical discomfort and fatigue arises during actual procedures. This can influence our results [14]. 
Third, the research group consisted of inexperienced participants without specific surgical skills. Because the effect of surgical skills on the reduction of stress is not known, it is hard to determine whether our research group is comparable with a population of laparoscopic surgeons. To a certain extent, the aforementioned factors may have affected our results. However, our results clearly indicate that improvement in manipulation and the introduction of a stereoscopic view in a comfortable and ergonomic workspace can contribute to an increased feeling of situational control and a decreased level of physical workload. In conclusion, the implementation of a robotic system for the execution of laparoscopic tasks in an experimental setup enhances performance and reduces cognitive stress levels as well as physical discomfort. 


\section{Appendix 1}

Subjective Mental Effort Questionnaire (SMEQ)

This graphic displays the amount of effort it took you to execute the task you have been working on.

Please score the amount of effort by marking one of the anchors on the vertical line here below.

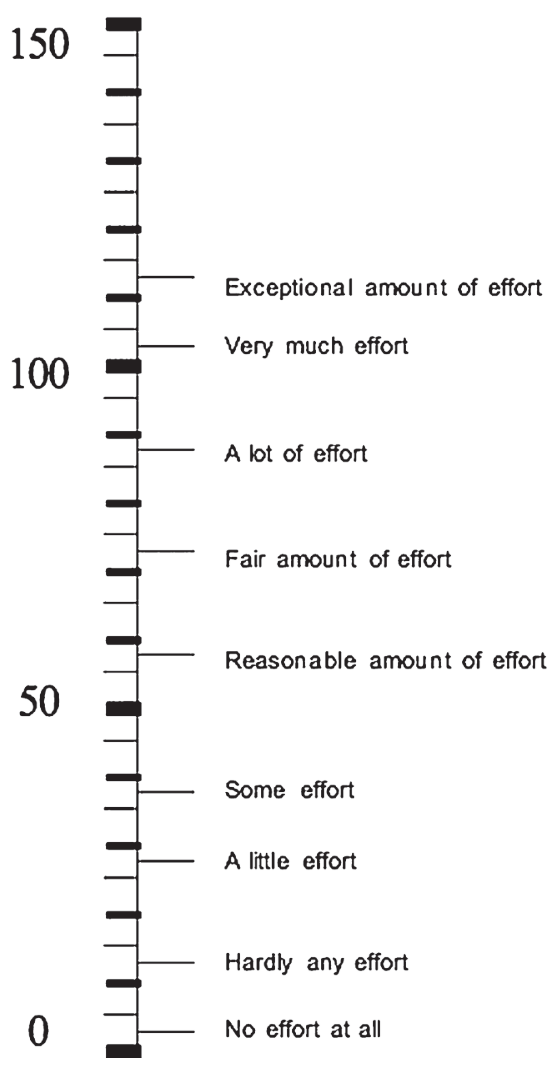




\section{Appendix 2}

Local Experienced Discomfort Scale (LED scale)

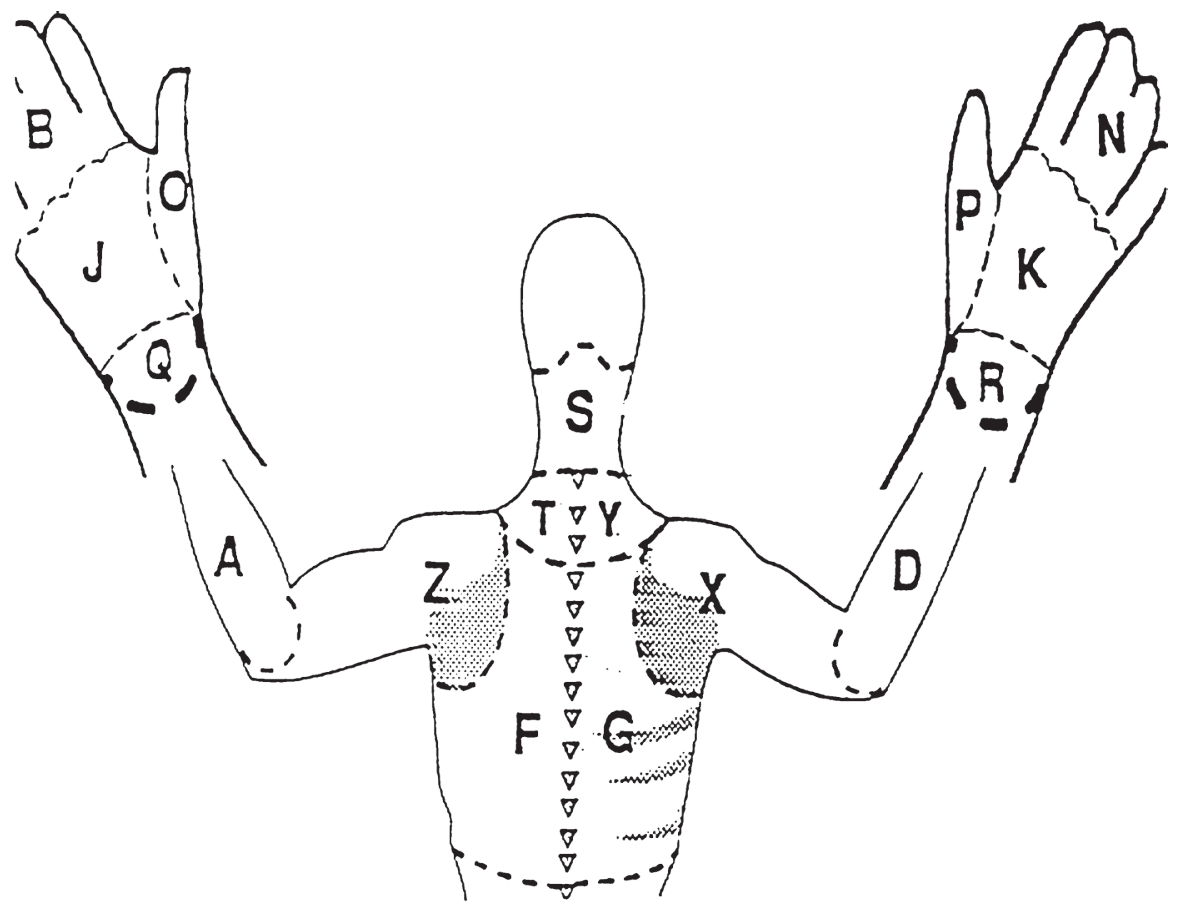

For each of the body parts indicated by the letters in the picture, please fill in a score as presented here below.

\section{Maximum}

Extreme amount of complaints

$$
9
$$$$
8
$$$$
\begin{aligned}
& 7 \\
& 6
\end{aligned}
$$$$
5
$$$$
4
$$$$
2
$$$$
1
$$

$1 / 2$ Hardly any complaints

0 No complaints at all Minimum

\section{Right side Left side}

$\begin{array}{ll}B \ldots & \mathrm{N} \ldots \\ \mathrm{J} \ldots & \mathrm{K} \ldots \\ \mathrm{O} \ldots & \mathrm{P}_{\text {wow }} \ldots \\ \mathrm{Q} \ldots & \mathrm{R} \ldots \\ \mathrm{A} \ldots & \mathrm{D} \ldots \\ \mathrm{Z} \ldots & \mathrm{X} \ldots \\ \mathrm{T} \ldots & \mathrm{Y} \ldots \\ \mathrm{S} \ldots & \mathrm{S} \ldots \\ \mathrm{F} \ldots & \mathrm{G} \ldots\end{array}$




\section{References}

1. Ballantyne GH, Hourmont K, Wasielewski A (2003) Telerobotic laparoscopic repair of incisional ventral hernias using intraperitoneal prosthetic mesh. JSLS 7:7-14

2. Berggren U, Gordh T, Grama D, Haglund U, Rastad J, Arvidsson D (1994) Laparoscopic versus open cholecystectomie:hospitalization, sick leave, analgesia, and trauma responses. Br J Surg 81:1362-1365

3. Berguer R (1998) Surgical technology and the ergonomics of laparoscopic instruments. Surg Endosc 12:458-462

4. Berguer R, Forkey DL, Smith WD (2001) The effect of laparoscopic instrument working angle on surgeons' upper extremity workload. Surg Endosc 15:1027-1029

5. Berguer R, Smith W (2006) An ergonomic comparison of robotic and laparoscopic technique: the influence of surgeon experience and task complexity. J Surg Res 134:87-92

6. Berne MR, Levy MN, Stanton BA, Koeppen BM (2006) Berne \& Levy principles of physiology, 4th edn. Elsevier Mosby, Philadelphia, PA, pp 383-385

7. Berntson GG, Cacioppo JT, Binkley PF, Uchino BN, Quigley KS, Fieldstone A (1994) Autonomic cardiac control: Psychological stress and cardiac response in autonomic space as revealed by pharmacological blockades. Psychophysiology 31: 599-608

8. Bowersox JC, Shah A, Jensen J, Hill J, Cordts PR, Green PS (1996) Vascular applications of telepresence surgery: initial feasibility studies in swine. J Vasc Surg 23:281-287

9. Corlett EN, Bishop RP (1976) A technique for measuring postural discomfort. Ergonomics 9:175-182

10. Emam TA, Hanna G, Cuschieri A (2002) Ergonomic principles of task alignment, visual display, and direction of execution of laparoscopic bowel suturing. Surg Endosc 16:267-271

11. Garcia-Ruiz A, Gagner M, Miller JH, Steiner CP, Hahn JF (1998) Manual vs robotically assisted laparoscopic surgery in the performance of basic manipulation and suturing tasks. Arch Surg 133:957-961

12. de Geus EJC, Willemsen GHM, Klaver CHAM, van Doornen LJP (1995) Ambulatory measurement of respiratory sinus arrythmia and respiration rate. Biol Psychol 41:205-227

13. Harris WS, Schoenfeld CD, Weissler AM (1967) Effects of adrenergic receptor activation and blockade on the systolic preejection period, heart rate, and arterial pressure in man. J Clin Invest 46:1704-1714

14. Johnston WKIII, Hollenbeck BK, Wolf JS Jr (2005) Comparison of neuromuscular injuries to the surgeon during hand-assisted and standard laparoscopic urologic surgery. J Endourol 19:377-381

15. Krzeminski K, Kruk B, Nazar K, Ziemba AW, Cybulski G, Niewiadomski W (2000) Cardiovascular, metabolic, and plasma catecholamine responses to passive and active exercises. J Physiol Pharmacol 51:267-278

16. Majeed AW, Jacob G, Reed MW, Johnson AG (1993) Laparoscopist's thumb: an occupational hazard. Arch Surg 128:357

17. Mezzacappa ES, Kelsey RM, Katkin ES (1999) The effects of epinephrine administration on impedance cardiographic measures of cardiovascular function. Intern J Psychophysiol 31:189-196

18. Miyamoto Y, Higuchi J, Abe Y, Hiura T, Nakazono Y, Mikami T (1983) Dynamics of cardiac output and systolic time intervals in supine and upright exercise. J Appl Physiol 55:1674-1681

19. Newlin DB, Levenson RW (1979) Pre-ejection period: measuring ß-adrenergic influences upon the heart. Psychophysiology 16:546-553

20. Nifong LW, Chu VF, Bailey BM, Maziarz DM, Sorrell VL, Holbert D, Chitwood WR Jr (2003) Robotic mitral valve repair: experience with the da Vinci system. Ann Thorac Surg 75:438-442

21. Nilsson G, Larsson S, Johnsson F (2000) Randomized clinical trial of laparoscopic versus open fundoplication: blind evaluation of recovery and discharge period. Br J Surg 87:873-878

22. Nio D, Bemelman WA, den Boer KT, Dunker MS, Gouma DJ, van Gulik TM (2002) Efficiency of manual vs robotical (Zeus) assisted laparoscopic surgery in the performance of standardized tasks. Surg Endosc 16:412-415

23. Patkin M, Isabel L (1995) Ergonomics, engineering and surgery of endosurgical dissection. J R Coll Surg Edinb 40(2):120-132

24. Prasad SM, Maniar HS, Soper NJ, Damiano RJ Jr, Klingensmith ME (2002) The effect of robotic assistance on learning curves for basic laparoscopic skills. Am J Surg 183:702-707 
25. Renda A, Vallancien G (2003) Principles and advantages of robotics in urologic surgery. Curr Urol Rep 4:114-118

26. Ruurda JP, van Vroonhoven ThMJ, Broeders IAMJ (2002) Robot-assisted surgical systems: a new era in laparoscopic surgery. Ann R Coll Surg Engl 84:223-226

27. Ruurda JP, Broeders IA (2003) Robot-assisted laparoscopic intestinal anastomosis. Surg Endosc 17(2):236241

28. Sherwood A, Allen MT, Fahrenberg J, Kelsey RM, Lovallo WR, van Doornen LJP (1990) Methodological guidelines for impedance cardiography. Psychophysiology 27:1-23

29. Stiff G, Rhodes M, Kelly A, Telford K, Armstrong CP, Rees BI (1994) Long-term pain: less common after laparoscopic than open cholecystectomy. Br J Surg 81:1368-1370

30. Svedenhag J, Martinsson A, Ekblom B, Hjemdahl P (1986) Altered cardiovascular responsiveness to adrenaline in endurance-trained subjects. Acta Physiol Scand 126:539-550

31. Vanek VW, Rhodes R, Dallis DJ (1995) Results of laparoscopic versus open cholecystectomy in a community hospital. South Med J 88:555-566

32. Willemsen GHM, de Geus EJC, Klaver CHAM, van Doornen LJP, Carroll D (1996) Ambulatory monitoring of the impedance cardiogram. Psychophysiology 33:184-193

33. Zijlstra FRH (1993) Efficiency in work behaviour: a design approach for modern tools. PhD thesis, Delft University of Technology. Delft, The Netherlands, Delft University Press 


\section{Chapter}

\section{Ergonomic assessment of the da Vinci console in robot-assisted surgery}

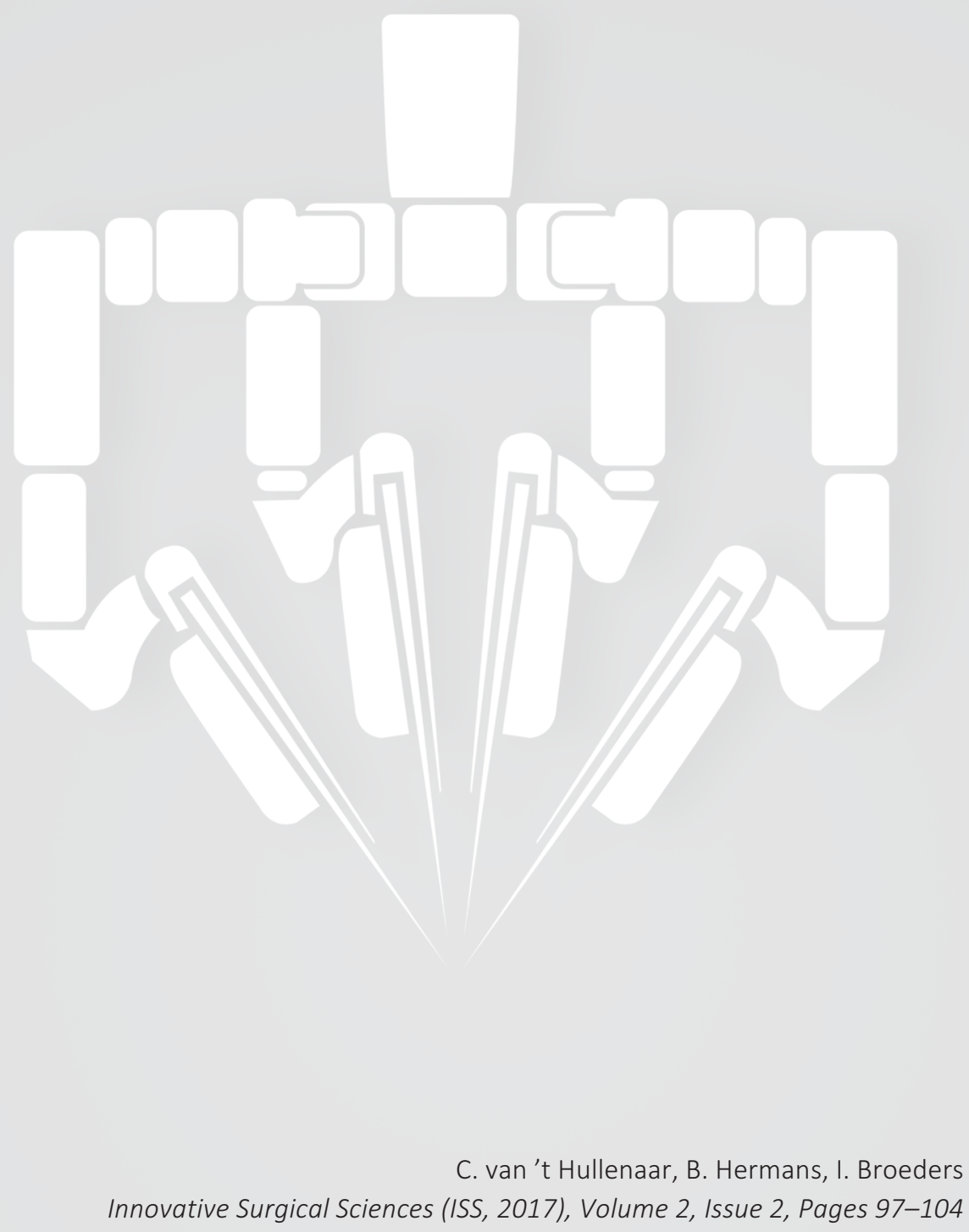




\section{Abstract}

Background: Robot-assisted surgery is considered to improve ergonomics over standard endoscopic surgery. Nevertheless, previous research demonstrated ergonomic deficits in the current console set-up.

Aim: This study was designed to objectively assess body posture in the da Vinci console during robot-assisted endoscopic surgery.

Methods: Multiple sagittal photographs from six physicians were taken during robotassisted procedures. Trunk, neck, shoulder, elbow, hip, and knee angles were calculated and compared to ergonomic preferable joint angles. A 2D geometric model was developed using individual anthropometrics. Optimal seat height, armrest height, and viewer height were calculated. These results were compared to the findings of the sagittal photographs.

Results: Mean joint angles show potentially harmful neck angles for all participants. Trunk angles vary between surgeons, from inadequate to correct. In short and very tall individuals, optimal armrest height is outside the adjustment range of the console.

Conclusion: The da Vinci Surgical System console seating position results in a nonergonomic neck and trunk angle. The developed geometric model revealed that armrest height has a limited adjustment range. Adjustments to the console and optimization of preoperative settings are goals to further improve ergonomics in robot-assisted surgery. 


\section{Introduction}

The first robotic systems in surgery were presentedmore than 30 years ago. An industrial robot arm, the PUMA (Westinghouse Electric, Pittsburgh, PA, USA), was used to manipulate surgical instruments for brain surgery in 1985. [1] In 1997, Himpens and Cadière performed the first robot-assisted cholecystectomy using the MONA system, a predecessor of the modern da Vinci Surgical System (Intuitive Surgical Inc., Sunnyvale, CA, USA). [1] The breakthrough of robot-assisted surgery occurred several years later when urologists began to use the da Vinci Surgical System for radical prostatectomy. [2] Nowadays, robot-assisted surgery has become the surgical golden standard for prostatectomy in many countries around the world. [3] The amount and diversity of surgical procedures wherefore robotic systems are applied is increasing every year. The main focus of robot-assisted surgery is to improve patient outcomes, but as a side effect surgeons report much better ergonomics for themselves compared to standard endoscopic surgery. This is especially the case for procedures in the small pelvis.

Standard endoscopic surgery is associated with major musculoskeletal discomfort. [68] Park et al. showed that $87 \%$ from 317 laparoscopic surgeons reported physical strain or symptoms of discomfort. [8] Manipulation of endoscopic instruments from a console provides a completely different perspective. The natural working axis is restored and the surgeon can work in a seated position with the arms on an armrest. [9-11]

Although robot-assisted surgery is considered to be more comfortable than endoscopic surgery, studies have shown that ergonomic deficits remain. [10-11] The optimal sitting position of the surgeon when using the da Vinci console is still unknown. The main goal of this study is to explore the body posture of the surgeon during robot-assisted surgery. Body joint angles were analysed and ergonomics in the da Vinci console were compared with preferable joint angles according to ergonomic guidelines.

\section{Methods}

\section{Participants}

Six physicians of the Meander Medical Centre (Amersfoort, the Netherlands) participated in this study. All participants were members of the hospital staff and were experienced in robot-assisted surgery (> 100 procedures each). The group consisted of three general surgeons, two urologists and a gynaecologist.

\section{Ergonomic evaluation}

Multiple sagittal plane photographs of the participants were taken during surgery. Trunk $(\Delta T)$, neck $(\Delta N)$, shoulder $(\Delta S)$, elbow $(\Delta E)$, hip $(\Delta H)$ and knee $(\Delta K)$ angles were calculated. A custom-made Matlab 2013a (The MathWorks, Natick, MA, USA) program was designed for this purpose. The participants limbs were traced by appointing hip, knee, ankle, shoulder, elbow and wrist.

$\Delta \mathrm{E}$ for example, is calculated between the appointed upper and lower arm segments. The trunk is appointed by tracing the physician's back with multiple points (red dots, figure 1). 
A straight line was fitted through these points using a standard least squares method. $\Delta T$ is then calculated between this linear fit and a vertical line perpendicular to the floor (dotted line, Figure 1). $\Delta \mathrm{N}$ is calculated between the linear fit of the trunk and a straight line between the point that represents the shoulder and the participants ear. All calculated joint angles are presented in Figure 1. Intra-observer variability was explored by analysing two participants ten times. Standard deviations of these data were subsequently calculated.

\section{Preferable joint angles}

The optimal joint angle range was extracted from the Rapid Upper Limb Assessment (RULA) tool. [14] The lowest RULA score (most optimal ergonomic position) is achieved when the joint angles are within the following ranges: $\Delta T=0-10^{\circ} ; \Delta N=0-10^{\circ} ; \Delta S=0-20^{\circ}$; $\Delta \mathrm{E}=60-100^{\circ}$. The angles $\Delta \mathrm{K}$ and $\Delta \mathrm{H}$ were defined to be optimal between $90^{\circ}$ and $110^{\circ}$ and between $90^{\circ}$ and $100^{\circ}$ respectively.

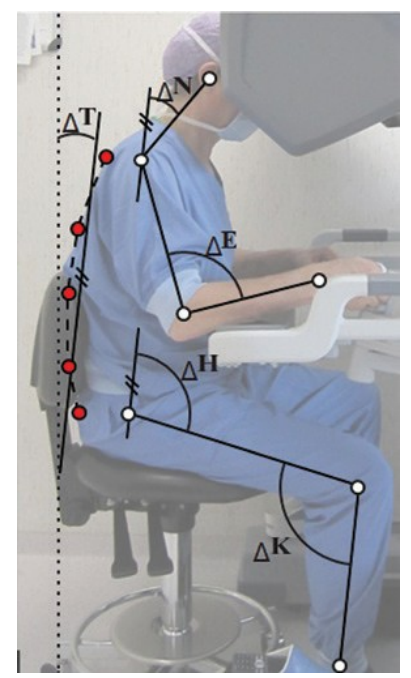

Figure 1: Representation of the angles calculated from the sagittal plane photographs: $\Delta T$ (trunk), $\Delta N$ (neck), $\Delta S$ (shoulder), $\Delta E$ (elbow), $\Delta H$ (hip), and $\Delta K$ (knee).

\section{Geometric model}

A two-dimensional (2D) geometric model was developed to evaluate the console ergonomics. Seat, armrest and viewer height can be calculated using a person's anthropometry and preferred joint angles. A schematic representation of this geometric model is shown in Figure 2. Seat, armrest and viewer height are calculated as follows: 
Seat height

Seat height can be calculated using equation 1 .

$$
A=\cos \left(\Delta K-90^{\circ}\right) \cdot L L
$$

$\Delta K$, Knee angle [degrees]; LL, Lower Leg [cm].

\section{Armrest height}

Optimal armrest height can be calculated by first calculating a person's shoulder height from seat level using equation 2 .

$$
B=\cos (\Delta T) \cdot T
$$

$\Delta \mathrm{T}$, Trunk angle [degrees]; T, Trunk [cm].

The difference in height between shoulder and elbow (D) is calculated using equation 3.

$$
D=\cos (\Delta S-\Delta T) \cdot U A
$$

$\Delta \mathrm{S}$, Shoulder angle [degrees]; $\Delta \mathrm{T}$, Trunk angle [degrees]; UA, Upper Arm [cm].

The elbow height can be calculated by using equation 4 . The elbow height is considered to be the optimal armrest height.

$$
\text { Armrest height }=A+B-D
$$

Viewer height

Similarly, the viewer optics height can be calculated using equation 5 .

$$
\text { Viewer height }=A+B+C
$$

Where $\mathrm{C}$ is the height difference between eyes and shoulder, calculated by equation 6 .

$$
C=\cos (\Delta N+\Delta T) \cdot S E
$$

$\Delta N$, Neck angle [deg]; $\Delta T$, Trunk angle [deg]; SE, Shoulder to Eye [cm]. 


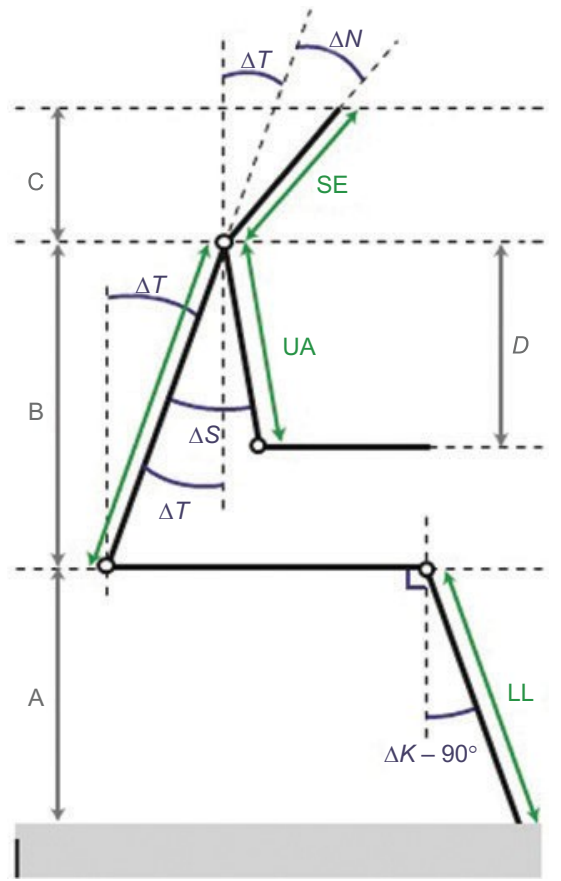

Figure 2: Schematic representation of the geometric model. LL, lower leg; T, trunk; SE, shoulder-eye; UA, upper arm.

To calculate the optimal settings, lengths of the upper arm, trunk, lower leg and the distance between shoulder and eye are crucial. For this study, anthropometric characteristics were extracted from the DINED 2004 database (TU Delft, the Netherlands). [13] To calculate optimal armrest height, anthropometric proportions were taken into account. For instance, when using an body length of $177 \mathrm{~cm}$ in the DINED database, the length of the lower leg length is $48 \mathrm{~cm}$. Dividing the lower leg length $(48 \mathrm{~cm})$ by the body length $(177 \mathrm{~cm})$, this results in an anthropometric proportion of 0.27 . With this number, the lower leg length of a person with a body length of $185 \mathrm{~cm}$ can be calculated. After using the equation $(0.27 \times 185)$, the exact lower leg length is determined $(50 \mathrm{~cm}) .[13]$

\section{Joint angles}

Optimal joint angles used in the geometric model were chosen with the adjustment ranges of the console in mind. The $\Delta T$ and $\Delta N$, for example, are both set to $20^{\circ}$ because of the viewer angle, which is at least $40^{\circ}$. Furthermore $\Delta K$ and $\Delta S$ are defined as $110^{\circ}$ and $40^{\circ}$ respectively. Even though our model is a $2 \mathrm{D}$ model, which does not include abduction in legs and arms, a shoulder abduction angle $\left(\mathrm{S}_{\mathrm{abd}}\right)$ of $20^{\circ}$ is taken into account for the optimal armrest height calculations. 


\section{Results}

\section{Ergonomic evaluation}

The data from all participants are shown in Appendix A. The mean joint angles of participant 1 and 2 are plotted in figure $3 a$ and $3 b$ respectively. The green shaded area in figure 3 represents the preferable joint angles according to ergonomic standards. It can be seen that these participants demonstrate angles that are potentially harmful. Especially trunk, neck and shoulder angles are out the 'safe' ergonomic zone.

Intra- and interobserver variation measurements resulted in standard deviations of less than $2^{\circ}$ for all angles in all participants.
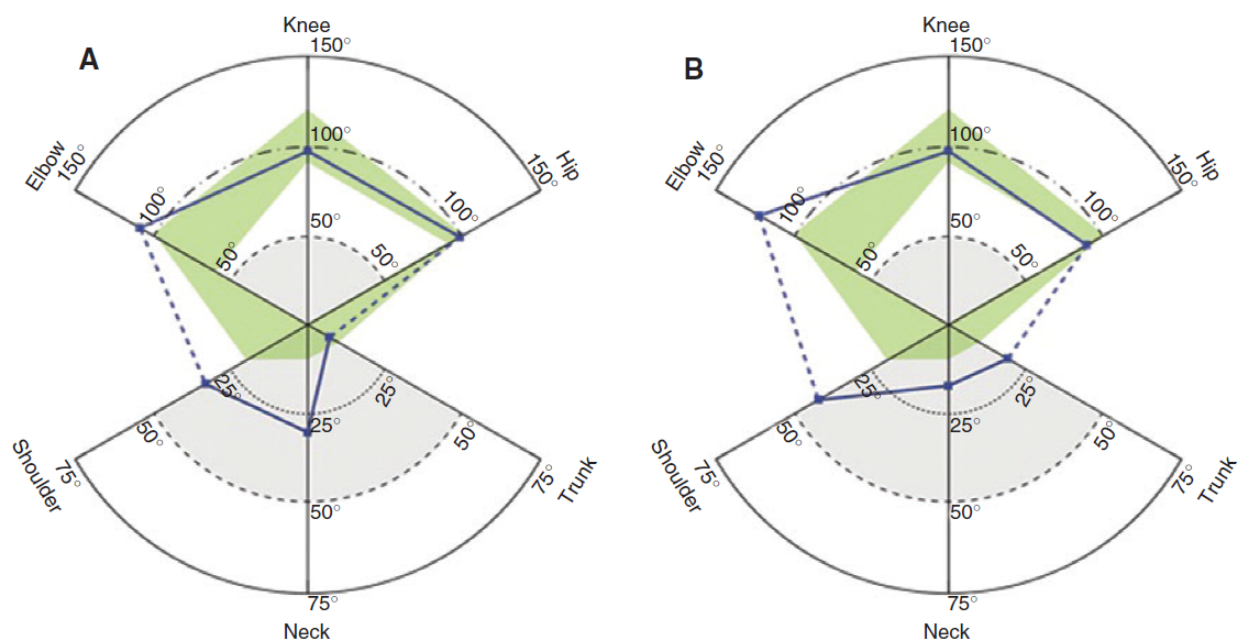

Figure $3(A)$ and (B) Mean joint angles of participants 1 and 2, respectively.

The green shaded area represents the preferable joint angle range. $\Delta K=90-110^{\circ}, \Delta H=90-100^{\circ}, \Delta T=0-10^{\circ}, \Delta N=0$ $10^{\circ}, \Delta S=0-20^{\circ}$ and $\Delta H=90-100^{\circ}$.

\section{Limitations of the console}

In the current console, the viewer angle can be adjusted between $40^{\circ}$ and $60^{\circ}$. The viewer height can be adjusted between 104 and $137 \mathrm{~cm}$. Armrest height can be changed between 74 and $84 \mathrm{~cm}$ from floor to top surface of the armrest.

The optimal armrest and viewer height for users with a stature between 140 and $200 \mathrm{~cm}$ and are shown in figure $4 \mathrm{a}$ and $4 \mathrm{~b}$. According to these data, persons smaller than $175 \mathrm{~cm}$ are not able to put the armrest at a desirable height. Optics height can vary from 104 to $137 \mathrm{~cm}$. This makes the optics height suitable for persons varying from $155 \mathrm{~cm}$ and $200 \mathrm{~cm}$ in length. 

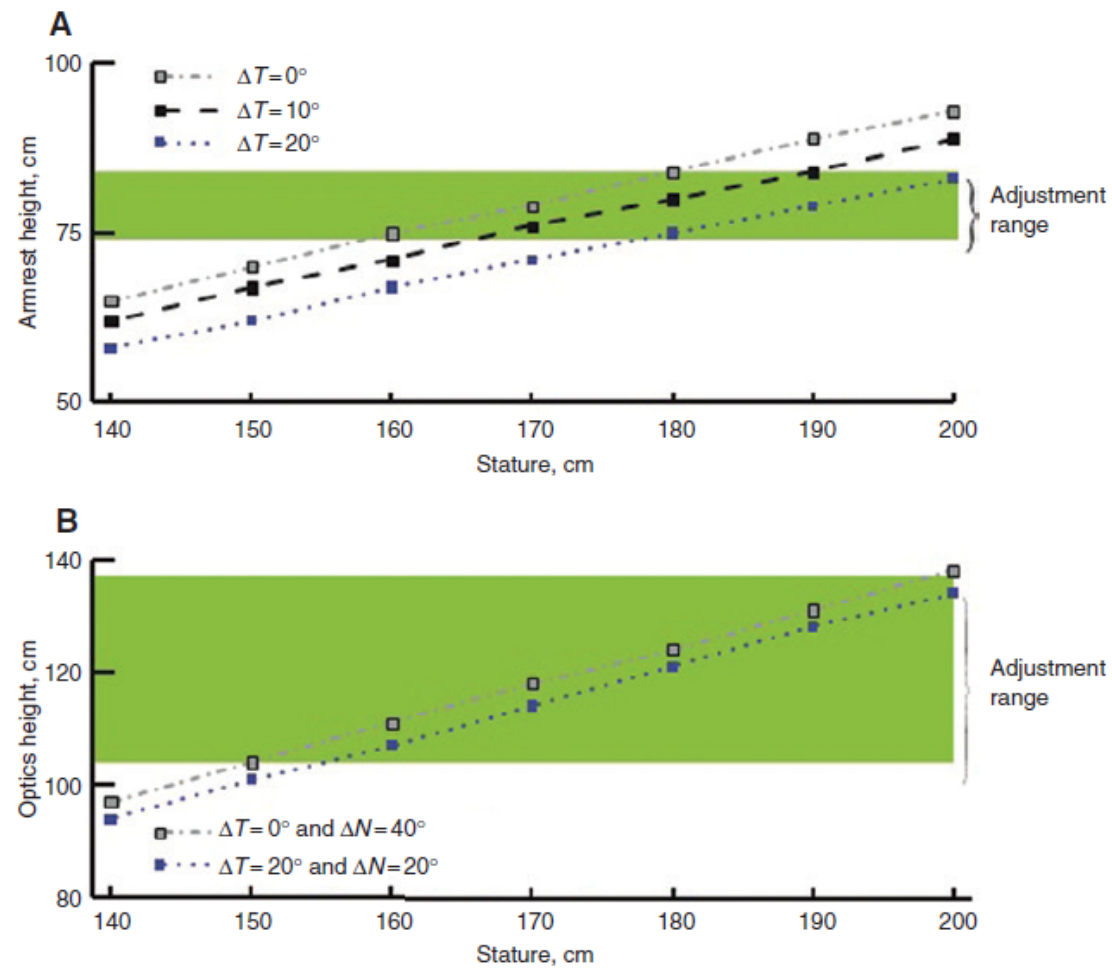

Figure 4: $(A)$ and (B) Optimal armrest and optics heights for different statures.

The optimal heights are calculated using the 2D geometric model, anthropometric data from the DINED database. The shaded area represents the adjusted range of the console.

\section{Discussion}

Potential harmful neck angles were recorded in all participants (see Appendix A). This is in accordance with previous publications. Bagrodia et al. compared open, laparoscopic and robot-assisted prostatectomy ergonomics using survey questions among 106 urologists. Although robot-assisted prostatectomy turned out to be the most ergonomic approach, a proportional amount of $23 \%$ of the participants reported physical strain during robot-assisted prostatectomy procedures. [10] Lawson et al. analysed musculoskeletal discomfort with the body part discomfort score (BPD) and ergonomics with the rapid upper-limb assessment tool (RULA). This study compared endoscopic with robot-assisted gastric bypass procedures. The conclusion of this paper states that robotassisted gastric bypass surgery results in less musculoskeletal stress to the upper extremities than the conventional endoscopic techniques. However, according to their BPD scores, current robot consoles induce a severe neck strain due to the viewing angle. Furthermore, their RULA scores revealed a less ergonomic position of the surgeons trunk during robot-assisted surgery. [11] A publication by Craven et al. studied robotic gynaecologic procedures and concluded that neck strain is an important issue in robotic 
surgery. Improvements in ergonomics by training and improvements of robotic workstations are suggested.[14] Finally, Lux et al. constructed several guidelines to optimize the ergonomic position of surgeon when using the da Vinci console. [15] When analysing the data of this research project in more detail, it is noticeable that the neck and trunk angle depend on each other. Participants with reasonable trunk angles, suffer from greater neck angles and vice-versa. Adding the actual values of the trunk and neck results in a total flexion angle of approximately 40 degrees in all participants. This is caused by the viewer angle of the console, which is at minimum 40 degrees.

Besides the neck and trunk angles, shoulder and elbow angles are also found to be non-ergonomic in all participants. Because the da Vinci console has an armrest, which supports the weight of the arm, the non-ergonomic angles of the shoulder probably have less impact.

In this study, user-specific optimal ergonomics are calculated with a geometric model. When taking the adjustment range of the console into account, the settings for the armrest height are not sufficient. This is displayed in figure 4a. Physicians with a length under $175 \mathrm{~cm}$ cannot place the armrest height to their optimal height. The adjustment range of the viewer height is much larger and allows acceptable ergonomics for users with a body length between $155 \mathrm{~cm}$ and $200 \mathrm{~cm}$ (figure $4 \mathrm{~b}$ ). Because of the limited adjustment range of the armrest height, it is not possible for users with a small posture to adjust the console to optimal settings.

An increase of the adjustment range for the armrest height and a decrease of the viewer angle in future consoles would support ergonomic settings in the current console design.

Several limitations of this study should be addressed. First, by using photographs from one direction (sideways), only sagittal ergonomic information was calculated. Since the neglected third dimension is considered to be less relevant for neck and trunk angles, the results of this study remain legitimate. The error in angle calculation might be larger for hip, knee, shoulder and elbow angles. This is caused by abduction in the upper arm and hip. The geometric model that was applied in this study, was corrected for an abduction of 20 degrees in the shoulder. By applying that formula, armrest height calculations were measured accurate.

Another factor is that the identification of anatomical landmarks on photographs comes with a certain level of inaccuracy. To cross check the reproducibility and accuracy of all calculated angles in this study, an intra-observer variability test was performed. This test revealed a standard deviation of less than 2 degrees for all angles. Therefore, the data derived from this study are considered to be reproducible and accurate.

Finally, personal console settings need to be addressed. In this study, there were no ergonomic instructions provided to the surgeons. This might have led to less optimal console statures (figure 5). With the geometric model described in this study, the optimal user settings for the individual surgeon can be determined. It is now possible to use this algorithm for advise on optimal console settings, chair height and distance to the console. 

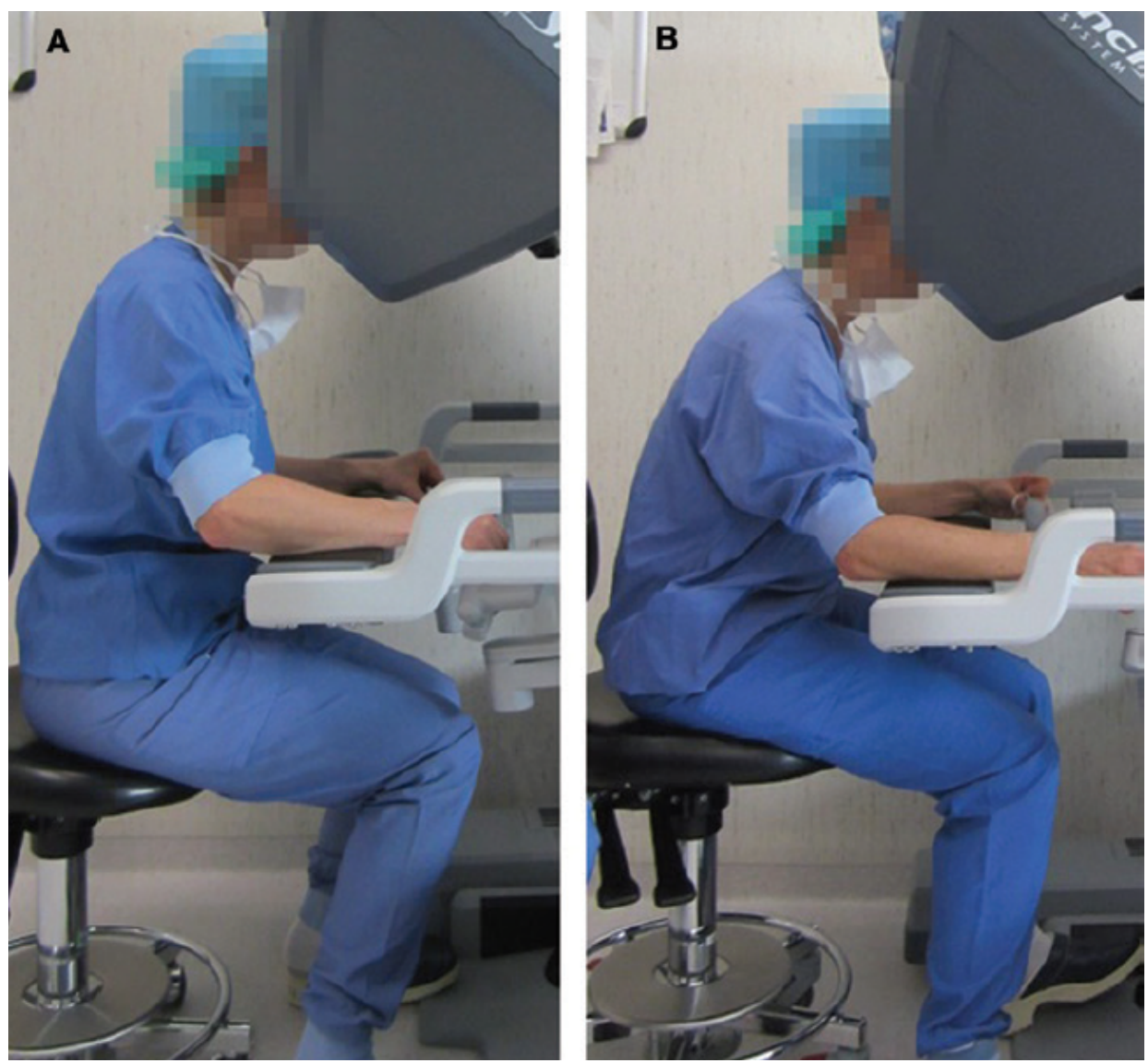

Figure 5: (A) Participant 3 photographed on the first day and (B) the same participant during a similar procedure on the next day. It can be seen that the participant did not adjust the chair height. This results in a less favourable ergonomic position

\section{Conclusion}

Robot-assisted surgery provides better ergonomics compared to standard endoscopic surgery. However, during robot-assisted surgery several physical complaints are reported. With this study, the surgeon's position in the da Vinci Surgical System console was analysed. After analysing the console posture of all study participants, a nonergonomic neck or trunk angle was found. These potential harmful angles are the result of the viewer angle of the console. The viewer of the console does not allow less than 40 degrees downward view and therefore it forces users to a flexed position of the spine. Furthermore, our geometric model indicates that the armrest height cannot be adjusted sufficiently. Future models of the robot console can take these findings into account. Ergonomic instructions derived from our geometric data, can help to optimise the seating 
position of the surgeon in the future. Our future research projects will also focus on the design of an optimal chair for the robot console. 


\section{References}

1. F Pugin, $P$ Bucher, and P Morel. History of robotic surgery: from AESO P and ZEUS to da Vinci. Journal of visceral surgery, 148(5 Suppl):e3-8, October 2011.

2. I Broeders. Robotics: The next step? Best practice \& research. Clinical gastroenterology, 28(1):225-232, February 2014.

3. S Chopra, Abhishek Srivastava, and Ashutosh Tewari. Robotic radical prostatectomy: The new gold standard. Arab Journal of Urology , 10(1):23-31, March 2012.

4. I Broeders and Sandy S Kalisingh. Handboek Endoscopische chirurgie. Bohn Stafleu van Loghum, Houten, 2009.

5. A Lanfranco, Andres E Castellanos, Jaydev P Desai, and William C Meyers. Robotic surgery: a current perspective. Annals of surgery, 239(1):14-21, January 2004.

6. F Cavallo, Andrea Pietrabissa, Giuseppe Megali, Elena Troia, Stefano Sinigaglia, Paolo Dario, Franco Mosca, and Alfred Cuschieri. Proficiency assessment of gesture analysis in laparoscopy by means of the surgeon's musculo-skeleton model. Annals of surgery, 255(2):394-8, February 2012.

7. K Miller, M. Benden, a. Pickens, E. Shipp, and Q. Zheng. Ergonomics Principles associated With Laparoscopic Surgeon Injury/ Illness. Human Factors: The Journal of the Human Factors and Ergonomics Society, 54(6):1087-1092, June 2012.

8. A Park, Gyusung Lee, F Jacob Seagull, Nora Meenaghan, and David Dexter. Patients benefit while surgeons suffer: an impending epidemic. Journal of the American College of Surgeons, 210(3):306-13, March 2010.

9. Van Veelen, Jakimowicz, and Kazemier. Improved physical ergonomics of laparoscopic surgery. Minimally invasive therapy \& allied technologies: MITAT : official journal of the Society for Minimally Invasive Therapy, 13(3):161-6, June 2004.

10. A Bagrodia and JD Raman. Ergonomic Considerations of Radical Prostatectomy: Physician Perspective of Open, Laparoscopic, and Robot-Assisted Techniques. Journal of Endourology , 23(4):627-633, 2009.

11. E. Lawson, Myriam J. Curet, Barry R. Sanchez, Rob Schuster, and Ramon Berguer. Postural ergonomics during robotic and laparoscopic gastric bypass surgery: a pilot project. Journal of Robotic Surgery, 1(1):6167, February 2007.

12. R Craven, Jason Franasiak, Prithima Mosaly, and Paola a Gehrig. Ergonomic deficits in robotic gynecologic oncology surgery: a need for intervention. Journal of minimally invasive gynecology , 20(5):648-55, 2013.

13. Technical University Delft. Dined, 2004. http://dined.io.tudelft.nl/dined, accessed: 20-04-2014

14. L McAtamney and E Nigel Corlett. RULA: a survey method for the investigation of work-related upper limb disorders. Applied ergonomics, 24(2):91-9, April 1993.

15. Lux MM, Matthew Marshall, Erdal Erturk, and Jean V Joseph. Ergonomic evaluation and guidelines for use of the daVinci Robot system. Journal of endourology / Endourological Society , 24(3):371-5, March 2010.

16. D D Harrison, S O Harrison, a C Croft, D E Harrison, and S J Troyanovich. Sitting biomechanics part I: review of the literature. Journal of manipulative and physiological therapeutics, 22(9):594-609, 1999. 


\section{Appendix A: Mean joint angles of all participants}

Mean joint angles of participant 1

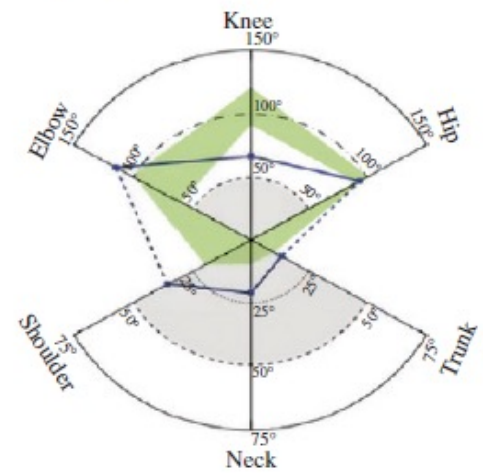

Mean joint angles of participant 3
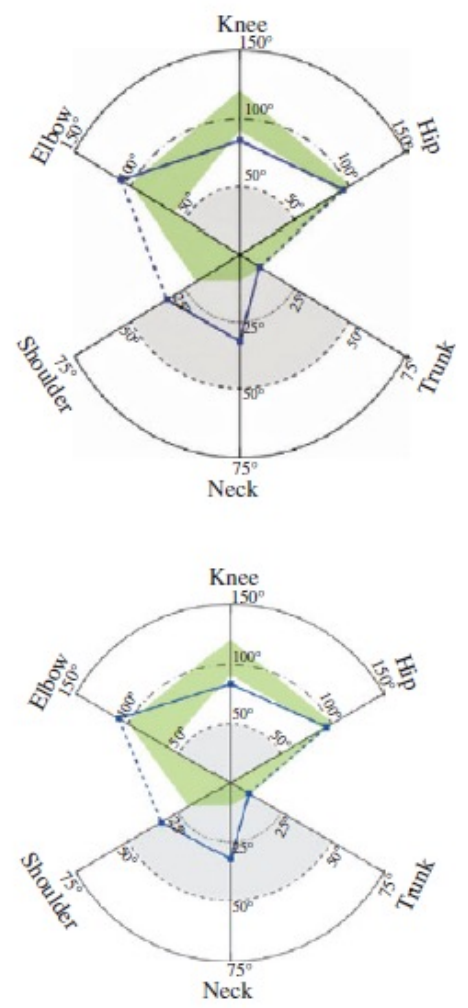

Mean joint angles of participant 5
Mean joint angles of participant 2

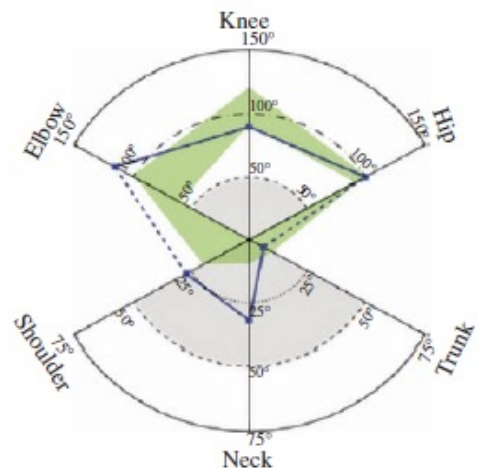

Mean joint angles of participant .
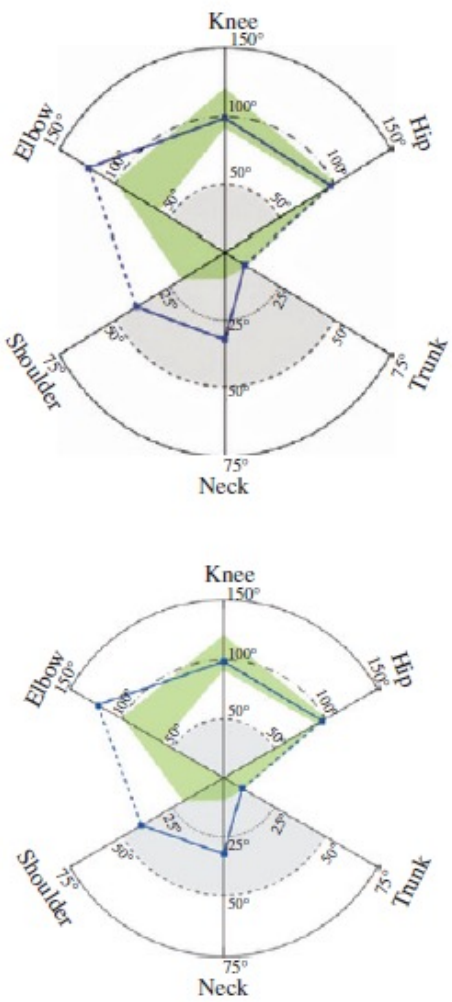

Mean joint angles of participant 6

Appendix A: Mean joint angles of all participants. The green shaded area represents the safe joint angles range according to the RULA score. $\Delta K=90-110^{\circ}, \Delta H=90-100^{\circ}, \Delta T=0-10^{\circ}, \Delta N=0-10^{\circ}, \Delta S=0-20^{\circ}$ and $\Delta H=90-100^{\circ}$. 



\section{Chapter}

\section{The design of a console chair by addressing the ergonomic deficits in robot assisted \\ surgery}

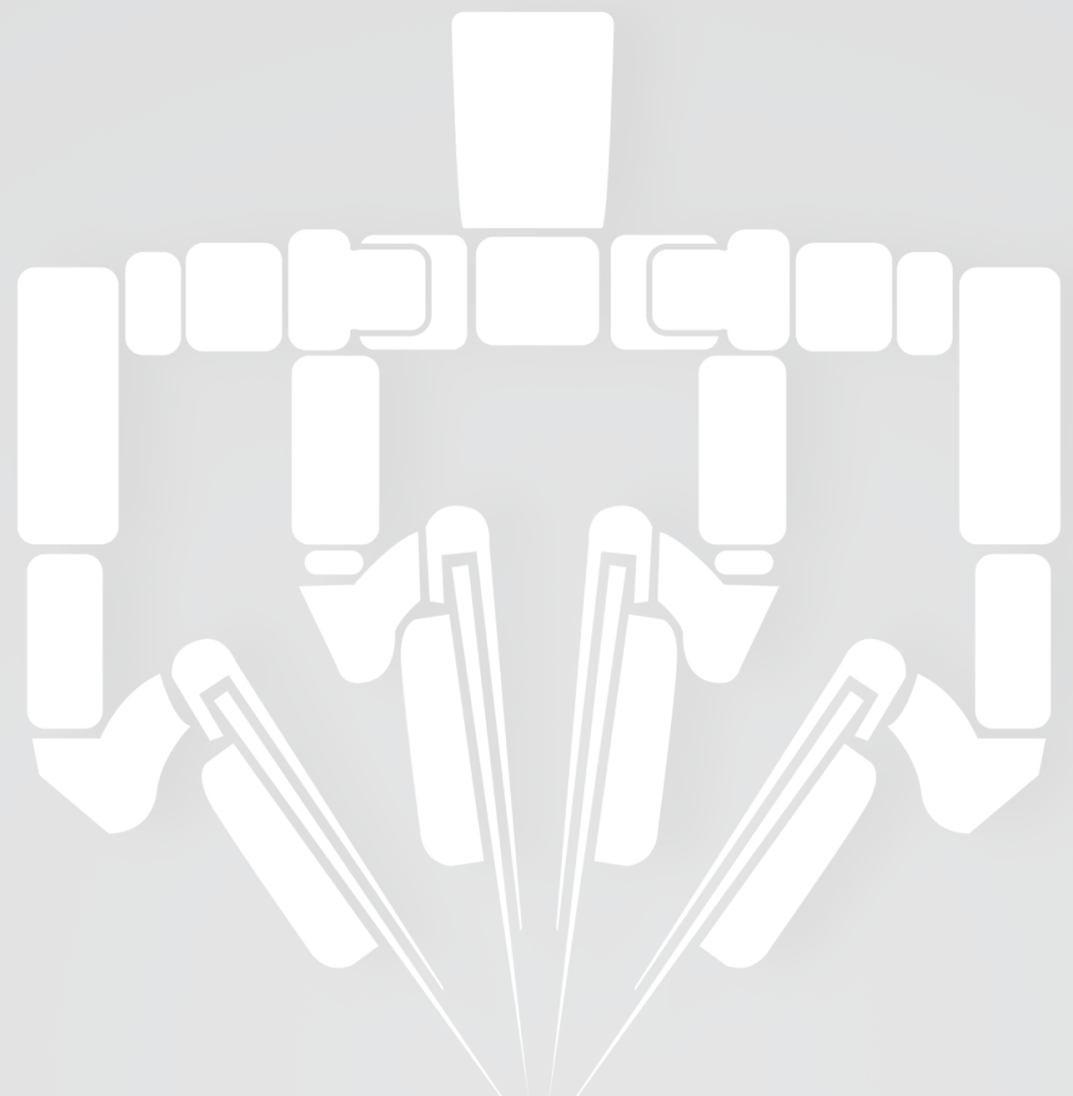

Research project performed at the Meander Medical Center, Amersfoort, The Netherlands H. Blankenvoort, C. van 't Hullenaar, I. Broeders Derived from Part 2 of the Master graduation thesis of $\mathrm{H}$. Blankenvoort, Faculty of Industrial Design Engineering, Twente University, The Netherlands 

Surgery in the twenty-first century is increasingly minimally invasive in its nature. Surgeons often work in standing postures, performing procedures that greatly tax the body. Robotic-assisted technology aims to change this. Firstly because it allows the surgeon to sit behind a console instead of having to stand for several hours on end. However, a chair is essential when sitting behind a console. The console for robot-assisted surgery does not come with a standard chair. That is why most hospitals use office chairs with their console. SCORE, a manufacturer of (medical) chairs and the Meander Medical Center joined forces in search of a more ergonomic and comfortable console chair. This allowed the designers to experience the live observation of robot-assisted procedures. This experience was essential to them gaining an insight into the ergonomic limitations of the console [1-19].

Insights from the overlapping fields of human anatomy, biomechanics and ergonomics were needed to equip console surgeons with a new design of chair that would support their forward-leaning posture. Human anatomy studies the structure of the human body - often in a static posture - while biomechanics studies the body in motion, applying mechanical principles to study the forces impacting its various constituent parts. Ergonomics on the other hand, deals with both posture and movement: it studies the human body performing tasks, analysing the interactions between the user and a product or tool. Interactions, in this sense, may require effort in both the physical and cognitive domain. Depending on the aim of an ergonomic analysis, as well as the domains taken into account, a product's impact on factors like health and safety risks, comfort, productivity and user-friendliness can be assessed.

Serious ergonomic deficits of the console have been identified in earlier research [20]. Surgeons often sit on the edge of their seats, enjoying a minimal supporting area of the seat pan, in turn, leading to peak pressures and instability. This posture enabled surgeons to still reach the floor and foot pedals. The chair's orientation also proved a problem. The frame's legs are often in the 'action zone' of a surgeon's feet - obstructing feet movements - and do not guarantee a centred orientation. An improved design may feature a frame that can be centred in front of the console and locked to a proper working distance setting. The console features integrated armrests, which offer the surgeon a welcome means of support. These armrests, however, interfere with the arm- and hand manipulations needed to perform the surgery. Surgeons were often seen to perform manipulations hovering above, or in front of the armrests, and so no longer using the support and unfavourably encumbering the arms. The console viewer also offers the head the option of resting against a pad somewhat, but most surgeons reported this as uncomfortable when used over a longer period of time.

Forward-leaning postures give people the opportunity to reach closer to their work both forward as well as downward, - which will sometimes be a necessity. Mandal distinguishes only two means of reaching over a surface while remaining seated, i.e. (A) hunching over, rounding the back, and (C) stretching in the knee joint while keeping the feet on the ground (see figure 1) [22]. Mandal doesn't address a third way one might 
achieve sufficient reach, i.e. active sitting via deliberate muscular effort (B). In robotassisted surgery, the limited adjustability of the console's armrests and viewer doesn't allow all surgeons to sit higher up on a tilted seat, whereby a combination of active sitting and seat pan tilt would allow all surgeons to improve their seated posture. Shorter surgeons may sit higher up, enjoying reduced pressure to their backs, by adopting a more open hip angle. Taller surgeons may sit on a more flattened seat pan, where they have to lean in more by decreasing their hip angle.

Still, maintaining such postures for any length of time requires considerable muscular effort, especially when performing surgical procedures over a period of several hours. That is why a type of support was needed that would only require a minimally-taxing posture to be maintained throughout the procedure.

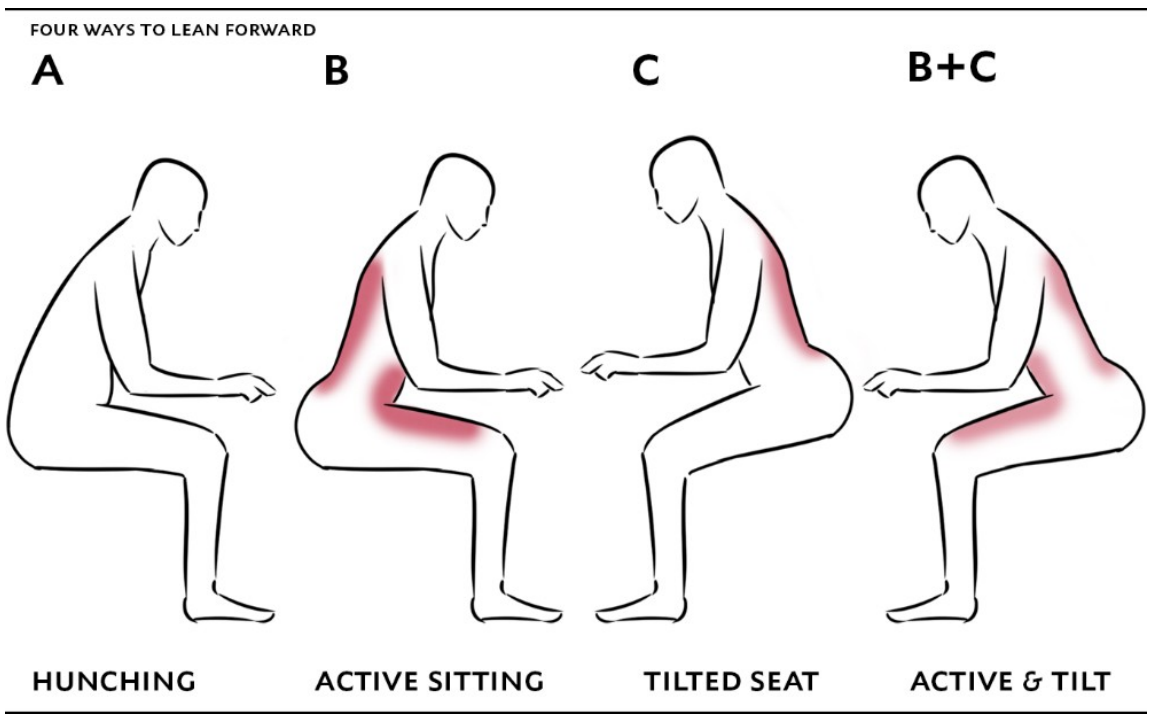

Figure 1. Four ways to lean forward (derived from Mandal's article) [22]

Several prototypes were made over the course of composing this thesis: four upholstered tilting seat pans, three sacral supports and three frames. Many of the elements used were components available from stock, such as gas lifts, castors and mechanisms that connect the seat and sacral support to the gas lift and which can be adjusted. Several frames were welded by hand on the factory floor in collaboration with the product development department at SCORE. Seat pans were made from wooden parts used in existing SCORE products, and were upholstered by a local upholsterer. The sprung hinges were made using torsion springs and sheet metal. All prototypes were brought to the Meander Medical Center for testing on multiple occasions. Operating theatre testing attended by surgeons generated very useful feedback. 
Several prototypes were tested. One of these had stronger, double winding torsion springs, a sacral support mechanism, a 'T-frame' with brakes and a central orientation system integrated. Wide chair legs would form bumpers that could ride up to the console's front edge, 'docking,' as it where, the frame to the console (Figure 2).

In order to allow the user to consistently achieve an ideal seat height, a proof ofconcept sensor solution was designed on an Arduino platform. Connecting a simple display and an ultrasonic range-finder, the functional distance could be programmed into the Arduino. A calculation tool, created for determining the ideal seat pan height, as well as console settings (armrests and viewer) pursuant to a surgeon's height, was developed. These settings could then be derived from a table displayed on the wall next to the console and compared with the height indicated on the display.

Based on three evaluation forms by surgeons that were interviewed, this revealed a good level of overall satisfaction. On most aspects, surgeons rated the final version of the prototype higher than the chairs currently in use, with most of these aspects also receiving a favourable rating. Sensor and calculator developments were not undertaken at SCORE, as this has been planned to be assigned to a number of the University of Twente's electrical engineering students. This will allow the development of a more robust electronic product, which would also be able to measure the console's settings (armrest and viewer height). As the project neared its end, an additional design phase was planned, that would address the potential of the seat pan and sacral support to other markets, and aimed to create a more appealing product, and would commence upon the project's conclusion. (Figure 3). Its aim was to create a more integrated design that combined the various functionalities (i.e. joining the stock mechanism, sacral support and the sprung hinge) into a single package.

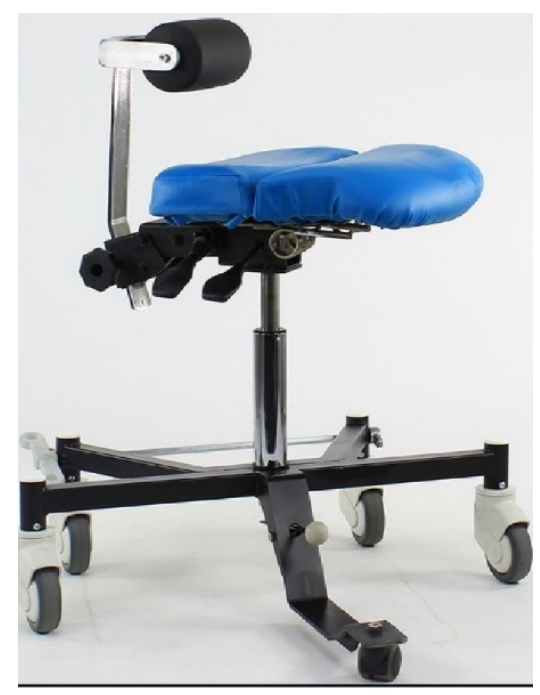

Figure 2. Prototype chair tested at the operating theatre 


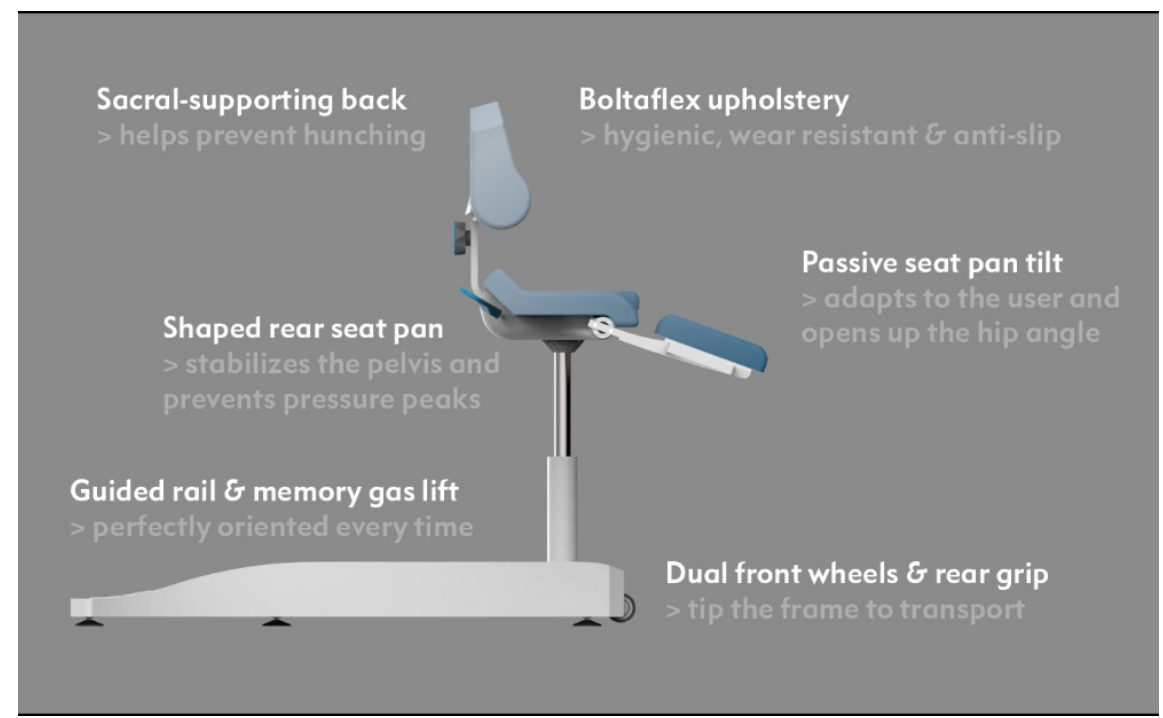

Figure 3. The chair's final prototype design 


\section{References}

1. Bendix, T, V Poulsen, K Klausen, and CV Jensen. 1996. "What Does a Backrest Actually Do to the Lumbar Spine?" Ergonomics 39: 533-542. doi:10.1080/00140139608964479.

2. Berkowitz, Shari. 20n. "Neutral Pelvis and Neutral Spine: What Are They and Why Do We Care?" The Vertical Workshop. https://theverticalworkshop.wordpress.com/2on/04/01/neutral-pelvis-and neutralspine-what-are-they-and-why-do-we-care/.

3. Bridger, R S, C Von Eisenhart-Rothe, and M Henneberg. 1989. "Effects of Seat Slope and Hip Flexion on Spinal Angles in Sitting." Human Factors 31: 679- 688. doi:10.1016/0003-6870(91)90179-L.

4. Broeders, Ivo a M J. 2014. "Robotics: The next Step?" Best Practice \& Research. Clinical Gastroenterology 28 (1) (February): 225-32. doi:10.1016/j.bpg.2013.12.001.

5. Brok, Hans. 2014. "Twente Krijgt Operatierobot." Tubantia, November 12.

6. Chau, Josephine Y., Hidde P. Van Der Ploeg, Jannique G Z van Uffelen, Jason Wong, Ingrid Riphagen, Genevieve N. Healy, Nicholas D. Gilson, et al. 2010. "Are Workplace Interventions to Reduce Sitting Effective? A Systematic Review." Preventive Medicine. doi:10.1016/j.ypmed.2010.08.012.

7. Congleton, J j, MM Ayoub, and J L Smith. 1985. "The Design and Evaluation of the Neutral Posture Chair for Surgeons." doi:10.n77/001872088502700507.

8. Corlett, E. N., and H. Gregg. 1994. "Seating and Access to Work." In Hard Facts About Soft Machines, edited by Rani Lueder and Kageyu Noro, 335-345. London: Taylor and Francis.

9. Corlett, E.N. 1999. "Are You Sitting Comfortably?" International Journal of Industrial Ergonomics. doi:10.1016/So169-8141(98)00083-3. 2006. "Background to Sitting at Work: Research-Based Requirements for the Design of Work Seats." Ergonomics 49: 1538-1546. doi:10.1080/00140130600766261.

10. Corlett, E.N., and J.A. Eklund. 1984. "How Does a Backrest Work?" Applied Ergonomics 15: m-n4. doi:10.1016/0003-6870(84)90282-5.

11. Cuddy, Amy. 2012. "Amy Cuddy: Your Body Language Shapes Who You Are." Www.ted.com. http://www.ted.com/talks/amy_cuddy_your_body_language_shapes_who_you_are.html.

12. Cuddy, Amy j, Caroline A Wilmuth, and Dana R Carney. 2012. "The Benefit of Power Posing Before a HighStakes Social Evaluation." http://dash.harvard.edu/handle/1/9547823.

13. Dunstan, David W., Bronwyn A. Kingwell, Robyn Larsen, Genevieve N. Healy, Ester Cerin, Marc T. Hamilton, Jonathan E. Shaw, et al. 2012. "Breaking up Prolonged Sitting Reduces Postprandial Glucose and Insulin Responses." Diabetes Care 35: 976-983. doi:ro.2337/dcn-1931.

14. Eger, Arthur 2007. Evolutionaire Productontwikkeling: Productfasen Beschrijven de Meest Waarschijnlijke Levensloop van Een Product. LEMMA.

15. Fuchs, Thomas, and Sabine C Koch. 2014. "Embodied Affectivity: On Moving and Being Moved." Frontiers in Psychology 5 (June) (January): 508. doi:10.3389/fpsyg.2014.00508. http://www.pubmedcentral.nih. gov/articlerender.fcgi?artid=4047516\&tool=pmcentrez\&rendertype=abstract.

16. Gray, H. 1919. Anatomy of the Human Body. The American journal of the Medical Sciences.

17. Haaksma, Ernst. 2007. "The Seated Man and Back Complaints." http://www.bqergonomics.eu/blobs/pdf/ Homosedens_EN.pdf.

18. Hamilton, Marc T., Genevieve N. Healy, David W. Dunstan, Theodore W. Zderic, and Neville Owen. 2008. "Too Little Exercise and Too Much Sitting: Inactivity Physiology and the Need for New Recommendations on Sedentary Behaviour." Current Cardiovascular Risk Reports. doi:ro.1007/s12170-008-0054-8.

19. Harrison, D.D., S.O. Harrison, A.C. Croft, D.E. Harrison, and S.J. Troyanovich. 1999. "Sitting Biomechanics Part I: Review of the Literature." Journal of Manipulative and Physiological Therapeutics 22 (9): 594-609. http://www.ncbi.nlm.nih.gov/pubmed/10626703.

20. C. van't Hullenaar, B. Hermans, I. Broeders Ergonomic assessment of the da Vinci console in robot-assisted surgery Innovative Surgical Sciences (ISS, 2017), Volume 2, Issue 2, Pages 97-104

21. Keegan, J J. 1953. "Alterations of the Lumbar Curve Related to Posture and Seating." The journal of Bone and joint Surgery. American Volume 35-A: 589- 603. 
22. Mandal, A C. 1981. "The Seated Man (Homo Sedens) the Seated Work Position. Theory and Practice." Applied Ergonomics 12: 19-26. doi:ro.1016/0003- 6870(81)90089-2.-.1987. "Balanced Sitting Posture on Forward Sloping Seat."

23. McMullin, David. 2000. "Een Schommelstoel Tegen Rugpijn." Delta Magazine TU Delft (29). http://delta.tudelft.nl/artikel/een-schommelstoel-tegen- rugpijn/4814.

24. Mischel, Nicholas A., Ida J. Llewellyn-Smith, and Patrick J. Mueller. 2014. "Physical (in)activity- Dependent Structural Plasticity in Bulbospinal Catecholaminergic Neurons of Rat Rostral Ventrolateral Medulla." Journal of Comparative Neurology 522: 499-513. doi:ro.roo2/cne.23464.

25. NASA. 1978. "Anthropometric Source Book Volume I: Anthropometry for Designers"

26. Noro, Kageyu, Tetsuya Naruse, Rani Lueder, Nobuhisa Nao-i, and Maki Kozawa. 2012. "Application of Zen Sitting Principles to Microscopie Surgery Seating." Applied Ergonomics 43: 308-319. doi:ro.ro16/ j.apergo.201r.06.006.

27. Riem, Pieter. 2013. "Project Innovatie Zadel Zit Score."

28. Riskind, john H. 1984. "They Stoop to Conquer: Guiding and Self-Regulatory Functions of Physical Posture after Success and Failure." Journal of Personality and Social Psychology. doi:ro.ro37/0022-3514.47.3.479.

29. Schünke, Michael, Erik Schulte, and Udo Schumacher. 2oro. Anatomische Atlas Prometheus - Algemene Anatomie En Bewegingsapparaat. Bohn Stafleu van Loghum.

30. Shusterman, Richard. 20II. "Muscle Memory and the Somaesthetic Pathologies of Everyday Life." Human Movement 12 (1) (January 1): 4-15. doi:ro.2478/vroo38-o II-ooo1-2 http://www.degruyter.com/view/ jjhumo.20ll.12.issue-1/v10038-0 II-0oo1-2/vroo38-0II-0001-2.xml.

31. Staarink, H.A.M. 2007. Zo Zit Het!. Assen: Koninklijke Van Gorcum BV. Steelcase. 2012. "Movement in the Workplace." http://360.steelcase.com/whitepapers/movement-in-the-workplacej.

32. Stevens, Edward Martin, Jerome Congleton, and Cordon Vos. 2004. "Design Guidelines and Evaluation of an Ergonomic Chair Feature Capable of Providing Support to Forward-Leaning Postures." Texas A\&M University.

33. Van der Doelen, L H M, MP Netten, R H M Goossens, and ER Y NES University of Oulu, Finnish Institute of Occupational Health, the Oulu University of Applied Sciences, University of Eastern Finland, and University of Lapland. 20II. "Tactile Feedback to Influence Sitting Behaviour during Office Work." In NES 2011 Wellbeing and Innovation Through Ergonomics, 380-385.

34. Van Deursen, Dirk L., Chris j. Snijders, jaap H. Van Dieën, Idsart Kingma, and Leo L j M Van Deursen. 2001. "The Effect of Passive Vertebral Rotation on Pressure in the Nucleus Pulposus." Journal of Biomechanics 34: 405-408. doi:ro.ro16/Soo21-9290(oo )002ro-4.

35. Van Deursen, Leo L., Jaap Patijn, John R. Durinck, Ruud Brouwer, Jeanne R. van Erven-Sommers, and Bernard j. Vortman. 1999. "Sitting and Low Back Pain: The Positive Effect of Rotatory Dynamic Stimuli during Prolonged Sitting." European Spine Journal 8 (3) (June 2): 187-193. doi:ro.roo7/soo5860050155. http://link.springer.com/ro.1007/soo5860050155.

36. Van Geffen, Paul, Jasper Reenalda, Peter H. Veltink, and B. F J M Koopman. 2010. "Decoupled Pelvis Adjustment to Induce Lumbar Motion: A Technique That Controls Low Back Load in Sitting." International journal of Industrial Ergonomics 40: 47-54. doi:10.1016/j.ergon.2009.08.015.

37. Vos, Cordon A., Jerome J. Congleton, J. Steven Moore, Alfred A. Amendola, and Larry Ringer. 2006. "Postural versus Chair Design Impacts upon Interface Pressure." Applied Ergonomics 3T 619-628. doi:10.1016/j.apergo.2005.09.002.

38. Whitcome, Katherine K, Liza J Shapiro, and Daniel E Lieberman. 2007. "Fetal Load and the Evolution of Lumbar Lordosis in Bipedal Hominins." Nature 450 (7172) (December 13): 1075-8. doi:10.1038/natureo6342. http://www.ncbi.nlm.nih.gov/pubmed/18075592.

39. Zacharow, Dennis. 1990. "The Problems with Lumbar Support." Physical Therapy Forum 9 (35). http://www.yogaback.com/The_YogaBack_for_Driving/problems-with lumbar-support.html 


\section{Part}

Advanced ergonomics in minimal invasive surgery 



\section{Chapter}

\section{Validation of ergonomic instructions in robot- assisted surgery simulator training}

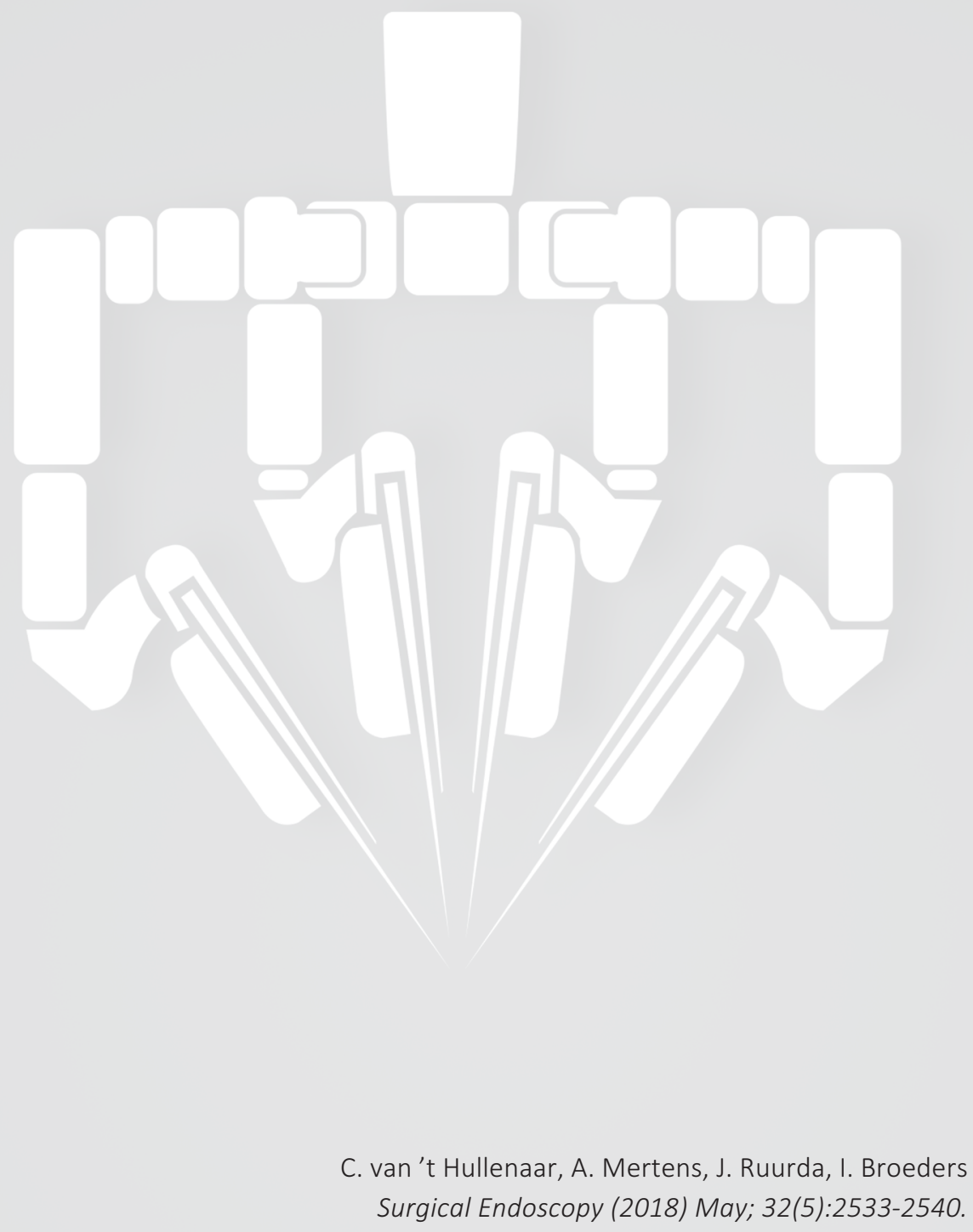




\begin{abstract}
Background/Aim Training in robot-assisted surgery focusses mainly on technical skills and instrument use. Training in optimal ergonomics during robotic surgery is often lacking, while improved ergonomics can be one of the key advantages of robot-assisted surgery. Therefore, the aim of this study was to assess whether a brief explanation on ergonomics of the console can improve body posture and performance.

Methods A comparative study was performed with 26 surgical interns and residents using the da Vinci skills simulator (Intuitive Surgical, Sunnyvale, CA). The intervention group received a compact instruction on ergonomic settings and coaching on clutch usage, while the control group received standard instructions for usage of the system. Participants performed two sets of five exercises. Analysis was performed on ergonomic score (RULA) and performance scores provided by the simulator. Mental and physical load scores (NASA-TLX and LED score) were also registered.

Results The intervention group performed better in the clutch-oriented exercises, displaying less unnecessary movement and smaller deviation from the neutral position of the hands. The intervention group also scored significantly better on the RULA ergonomic score in both the exercises. No differences in overall performance scores and subjective scores were detected.

Conlusion The benefits of a brief instruction on ergonomics for novices are clear in this study. A single session of coaching and instruction leads to better ergonomic scores. The control group showed often inadequate ergonomic scores. No significant differences were found regarding physical discomfort, mental task load and overall performance scores.
\end{abstract}


The introduction of robot-assisted surgery has led to major changes in several surgical specialities. With a current number of more than 3800 robot units installed worldwide, the system has proved to be an important tool that surgeons worldwide are widely adapting. One of the key features that the robot system can offer for the surgeon is optimized ergonomics. The surgeon is seated on a chair, while the arms are supported by an armrest. Compared to conventional laparoscopic surgery, this can lead to reduced muscle strain on the legs, shoulders and back.

With the introduction of robot systems, the focus is primarily on patient outcomes in relation to hospital expenses. Most of the time, little attention is paid to the comfort and ergonomics of the surgeon.

The robot system offers several opportunities to improve the ergonomic position of the surgeon in the console. The height of the chair, the level of the armrests, the height and angle of the view panel and the position of the foot pedals all influence body posture. Also the ability to use the clutch is of major importance. While pushing the clutch button, the console handgrips are temporarily uncoupled from the instruments. With the correct use of this clutching option, it is possible to move the instruments further into the distance without stretching or spreading the hands apart.

In current practice, there is not much attention for ergonomics when surgeons start with their initial experience in robot-assisted surgery. Implementing ergonomics in early training might stimulate awareness and improve ergonomic gain. Earlier studies demonstrate that novices use the clutch less often than experts (Raza et al. [2]). Incorrect use of the clutch can result in straining body posture because of overexertion of the arms, wrists or shoulders. Frequent and correct use of the clutch can, therefore, improve optimal ergonomic posture. The aim of this study was to investigate the effect of instructions in clutch usage and posture on performance and ergonomics in robotassisted surgery.

\section{Materials and methods}

A comparative study with novices in robotic surgery was conducted. All participants provided informed consent. At study entry, subjects were randomized into two groups by a randomisation list generated online (Sealed Envelope Ltd [3]). The goal of this study was to assess whether a brief ergonomic instruction, combined with a short coaching session on clutch usage, could provide an increase in ergonomics. All participants consecutively completed two different exercises. Both exercises were repeated five times by each participant.

Scores provided by the simulator system were collected to monitor performance during the training program. Also, an analysis of secondary outcomes for subjective ergonomic and workload scores was performed. Video analysis of every participant was carried out to score ergonomic outcomes objectively. 
Group A (control group) received the standard instructions for safe usage of the da Vinci console and da Vinci simulator, corresponding with the standard training regimen (Appendix 1).

Group B (intervention group) received the same standard instructions (Appendix 1), complemented with a short written guide on correct ergonomic adjustment of the console and seat (Appendix 2). Ensuing, the correct usage of the clutch controls was explained. After reading the written instruction, subjects received a brief instruction on ergonomic usage of the system. Additionally, during the first exercise, group B received verbal coaching regarding posture and correct usage of the clutch.

Optimal ergonomic settings of the console and chair were obtained by instructing subjects to sit with their feet supported, a straight back, the neck in line with the spine and the arms supported by the armrest. The console screen was set in an optimal position to prevent bending of the neck as much as the system allows. Figure 1 represents the posture of a participant before instruction, Figure 2 shows the posture after instructions.

Instructions on clutch usage were based on the observation that removing the arms from the armrest of the console is the start of a cascade of movements. Ultimately, this leads to overexertion of the arms and rotation of the trunk. This observation is in line with the research of Yang et al. [4], who proved the predictive value of armrest load on ergonomics. To this end, subjects were instructed to use the clutch when they needed to move their arms from the armrest to complete the exercise.

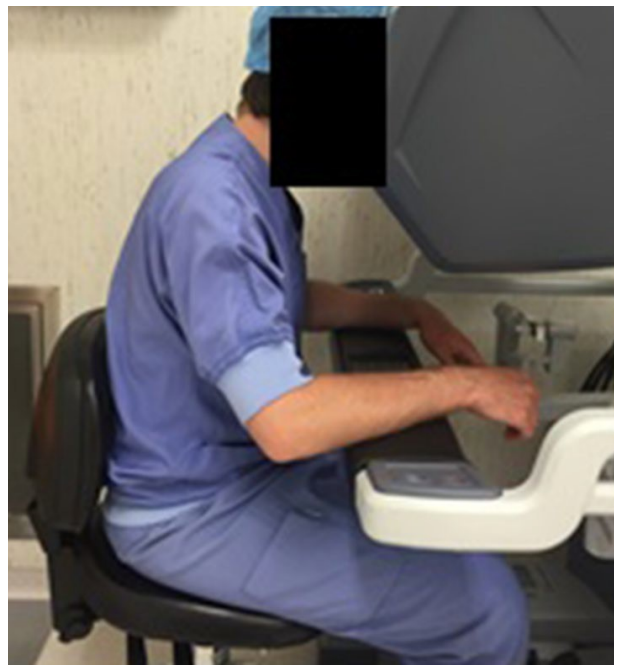

Figure 1 Posture before instruction

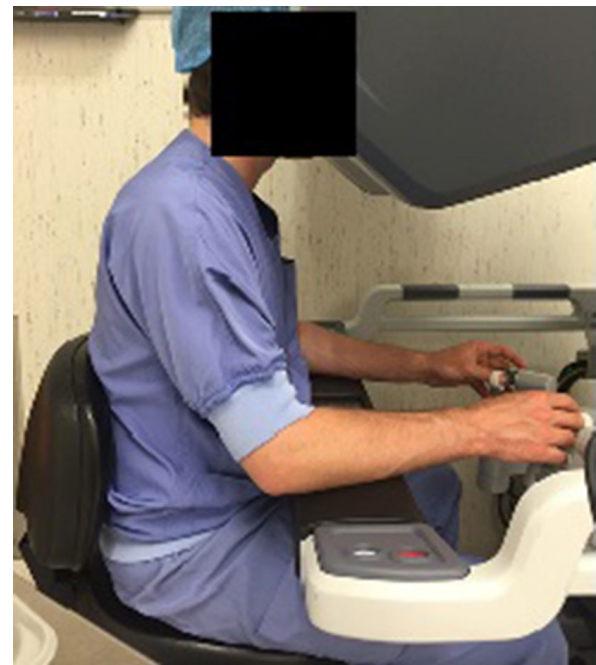

Figure 2 Posture after instruction

\section{Exercise 1: scaling}

Two training exercises were selected from the curriculum of the Intuitive Surgical skills simulator SM3000. These exercises reflect general skills in robot-assisted surgery and are 
part of most training regimens. The 'Scaling' training exercise (Figure 3) intends to train users in clutch control and camera handling. This is achieved by touching different targets with a cauterizing hook in a large workspace. The scaling exercise calls for a frequent use of the clutch, to prevent the subject from overreaching. When participants do not use the clutch and camera efficiently they have to reach further forward causing a suboptimal posture.

Exercise 2: needle targeting

The 'needle targeting' exercise (Figure 4) is designed to assess precision manipulation. This is practised by passing a needle through a bulls-eye into a target. This calls for precise manipulation of the instruments as well as the camera.

\section{Evaluation}

Differences in overall score, master workspace range, economy of motion and time to complete the exercise were extracted from the data provided by the skills simulator. Overall score is a weighted score combining all data. Master workspace range is defined as the maximum deviation of the hands from the neutral starting position, while moving instruments. As mentioned in the methods section of this article, large movements of the hands will result in inadequate posture. Hence, this is considered to be the most relevant outcome parameter in ergonomic evaluation. Economy of motion is the distance travelled by the instruments, where a lower value indicates more efficient instrument handling.

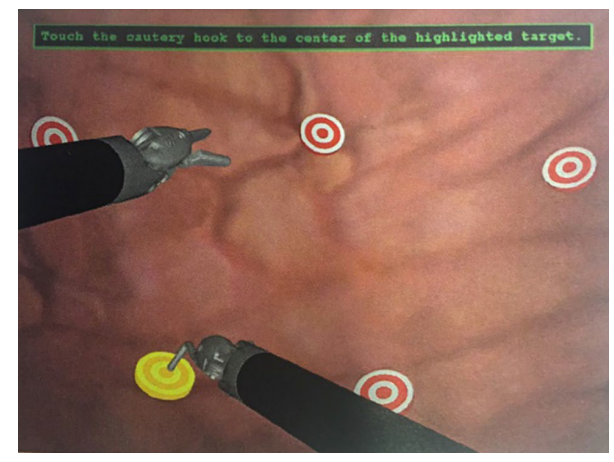

Figure 3 Scaling exercise

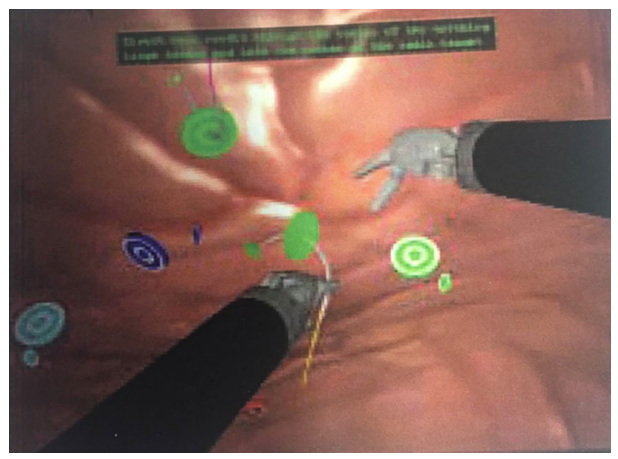

Figure 4 Needle targeting exercise

All subjects were evaluated using the Rapid Upper Limb Assessment score (RULA), an ergonomic scoring system designed to objectively assess the posture of the subject. The scores are based on angle measurements of various joints. This was achieved using sagittal video recordings. Every 30 seconds a snapshot was analysed using the RULA scoring system. The RULA score sheets were analysed by two independent observers. 
After five repetitions of each exercise, all subjects completed questionnaires on physical and mental demand (Local Experienced Discomfort scale [6], and NASA-TLX task load scale $[7,8]$.

The local experienced discomfort scale allows subjects to express the extent and location of physical discomfort on a ten-point scale. The NASA-TLX task load scale provides a multidimensional self-report questionnaire including six items: mental demand, physical demand, temporal demand, effort, frustration and performance. These scores are averaged and resulted in a score ranging between 0 and 100. A higher score indicates a higher workload.

A pilot study was performed with four subjects. Using these data, a power analysis was carried out. This indicated that for the main outcome measure "master workspace range", 13 subjects per group are required to have a $90 \%$ chance of detecting a decrease in master workspace range (significance at $5 \%$ ).

\section{Statistical analysis}

All data were processed and analysed using the IBM Statistical Package for Social Sciences, version 23.0 for Macintosh (SPSS, Chicago, IL).

Independent $t$ test was performed on baseline characteristics. A repeated-measure ANOVA with between-subject analysis was performed to detect differences between the two groups where applicable. Additionally, an independent samples $t$ test was performed on RULA, LED and NASA- TLX scores. A $P$ value of $<0.05$ was considered statistically significant. 
Table 1 Baseline table

\begin{tabular}{|c|c|c|c|c|c|c|}
\hline & $\begin{array}{l}\text { Control } \\
(n=13)\end{array}$ & & & $\begin{array}{l}\text { Inter- } \\
\text { vention } \\
(n=13)\end{array}$ & & $p$ value* \\
\hline \multirow[t]{2}{*}{ Sex (\%male) } & Male & 7 & $53.8 \%$ & 7 & $53.8 \%$ & 1.000 \\
\hline & Female & 6 & & 6 & & \\
\hline Age & Mean & 23.8 & & 24.85 & & 0.625 \\
\hline \multirow[t]{2}{*}{ Experience (robot-assisted surgery, \%yes) } & No & 13 & & 11 & & \\
\hline & Yes & 0 & $0.0 \%$ & 2 & $18.2 \%$ & 0.165 \\
\hline \multirow[t]{2}{*}{ Experience (laparoscopic surgery, \%yes) } & No & 12 & & 12 & & \\
\hline & Yes & 1 & $8.3 \%$ & 1 & $8.3 \%$ & 1.000 \\
\hline \multirow[t]{2}{*}{ Dominant hand (\%left) } & Right & 12 & & 10 & & \\
\hline & Left & 1 & $7.7 \%$ & 3 & $23.1 \%$ & 0.298 \\
\hline \multirow[t]{2}{*}{ Stage of training } & Intern & 7 & & 7 & & \\
\hline & Resident & 5 & & 5 & & \\
\hline
\end{tabular}

*Statistical significance was determined using the independent samples $t$ test

Table 2 Results scaling exercise

\begin{tabular}{llll}
\hline & Control Mean (SD) & Intervention Mean (SD) & $\begin{array}{l}P \text { value* } \\
P(95 \% \mathrm{Cl})\end{array}$ \\
\hline Overall score & $76.88(10.95)$ & $80.12(6.01)$ & $0.358(-10.40,3.90)$ \\
Master workspace range & $11.57(1.11)$ & $9.18(0.98)$ & $0.000(1.54,3.24)$ \\
Economy of motion & $302.32(62.94)$ & $249.08(29.55)$ & $0.013(12.56,93.92)$ \\
Time to complete & $154.77(24.00)$ & $184.31(28.31)$ & $0.008(-50.80,-8.30)$ \\
\hline
\end{tabular}

*Statistical significance was determined using the independent samples $t$ test

\section{Results}

Twenty-six subjects were included in total. As shown in Table 1, there were no significant demographic differences between the two groups. In each group, one participant had a small amount of laparoscopic. In the intervention group, two participants had limited experience using the robot system. This experience was limited to performing minimal tasks under direct supervision.

\section{Exercise 1: scaling}

The results of the scaling exercise are shown in Table 2. With regard to master workspace range (MWR), the intervention group performed much better with a significantly lower MWR (9.18 versus 11.57). That means that the clutch is correctly used and hand movements from the baseline are limited. When applying the repeated-measure ANOVA, the both groups showed a comparable improvement in MWR (Figure 5). 


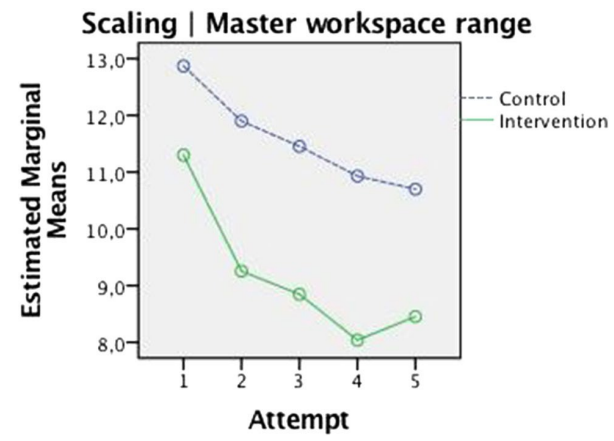

Figure $\mathbf{5}$ Scaling- master workspace range

In the intervention group, significantly lower economy of motion (EOM) was found ( $P=$ 0.013). In that group, less movement was necessary to complete the exercise, with a higher efficiency in movements. Additionally, the intervention group has a much lower standard deviation compared to the control group, indicating a more uniform performance after the instructions. Repeated-measure ANOVA showed no significant difference between the two groups (Fig. 6).

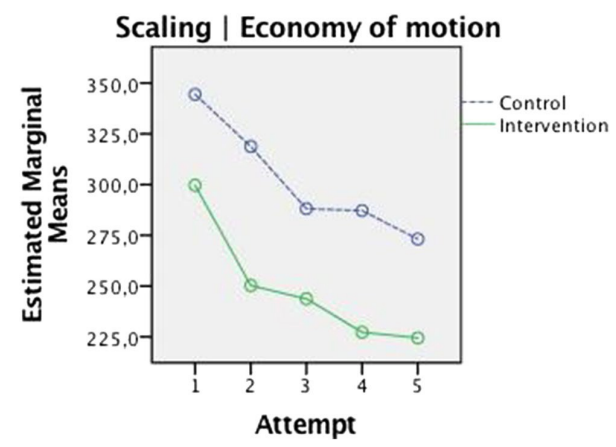

Figure 6 Scaling- economy of motion

No significant differences were detected in the mean overall score (MOS) $(P=0.358)$. Overall performance is equal (Figure 7). Regarding time to complete the exercise, the intervention group needed more time to complete the exercise $P=0.008$. Both groups improved their time scores equally, no significant differences were found in the repeatedmeasure ANOVA (Figure 8). Both the overall RULA score for the left $(P<0.001)$ and the right $(P<0.001)$ body half were significantly better in the intervention group. The RULA scores for separate body parts were also analysed. This showed a difference in the right upper $\operatorname{arm}(P=0.006)$, right lower arm $(P=0.002)$, neck $(P<0.001)$ and trunk $(P<0.001)$. 


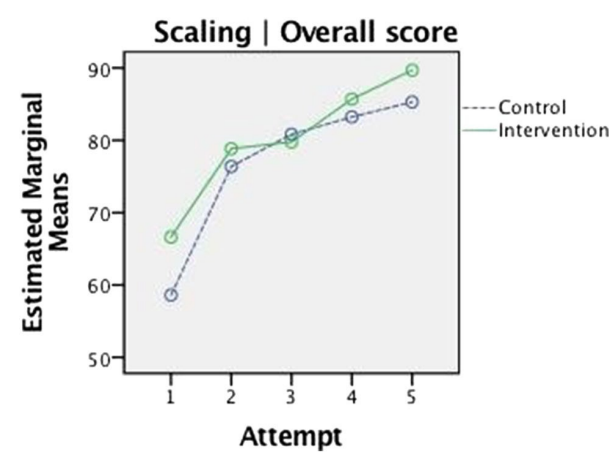

Figure 7 Scaling: overall score

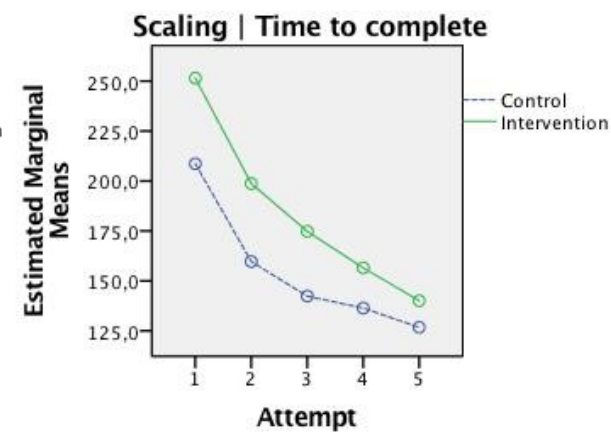

Figure 8 Scaling: time to complete

Concerning LED scores and NASA-TLX scores, no significant differences between both groups were found. Complaints such as muscle strain were reported to be low in both groups.

\section{Exercise 2: needle targeting}

A summary of the results of the needle targeting exercise is shown in Table 3. In exercise 2 , no differences between the groups in mean MWR were found. Also, repeated-measure ANOVA did not show any significant difference (Figure 9). No differences between the groups in mean EOM was found. Repeated-measure ANOVA reports a significant difference between the groups, $F(4,96)=3.355, P=0.010$. This means that a faster improvement in the EOM was displayed in the intervention group (Figure 10).

Regarding the MOS, no statistically significant difference was found between the groups. However, when repeated-measure ANOVA is analysed, this leads to a significant difference; $F(4,96)=4.443, P=0.002$. A faster improvement in overall score is seen in the intervention group after receiving ergonomic and clutching instructions (Figure 11).

There was no absolute difference in time needed to complete the exercise between both groups. When analysing repeated-measure ANOVA with Greenhouse-Geisser correction, this shows a faster improvement in the intervention group, $F(4,96)=4.414$, $P=0.013$ (Fig. 12). There were significant differences between both groups in the RULA score. Both the overall RULA score for the left $(P<0.001)$ and the right $(P<0.001)$ body half were much better in the intervention group. Looking at scores for separate body parts, there was a significant difference in the left lower arm $(P=0.041)$, neck $(P<0.001)$ and trunk $(P<0.001)$. Similar to the first exercise ('Scaling'), this displays a better posture after the instructions were provided. Participants did not report higher stress levels or muscle strain, as measured by the LED and NASA-TLX scores. Also during this exercise, complaints such as muscle strain were reported to be low. 
Table 3. Results needle targeting exercise

\begin{tabular}{llll}
\hline & Mean $(\mathrm{SD})$ & Mean $(\mathrm{SD})$ & $P(95 \% \mathrm{Cl})$ \\
\hline Overall score & $77.48(14.00)$ & $79.78(11.57)$ & $0.651(-12.71,8.09)$ \\
Master workspace range & $6.04(1.32)$ & $5.93(0.85)$ & $0.799(-0.79,1.01)$ \\
Economy of motion & $345.31(105.48)$ & $321.44(70.63)$ & $0.504(-48.80,96.53)$ \\
Time to complete & $251.16(68.92)$ & $276.92(82.85)$ & $0.397(-87.45,35.93)$ \\
\hline
\end{tabular}

Needle targeting | Master workspace range

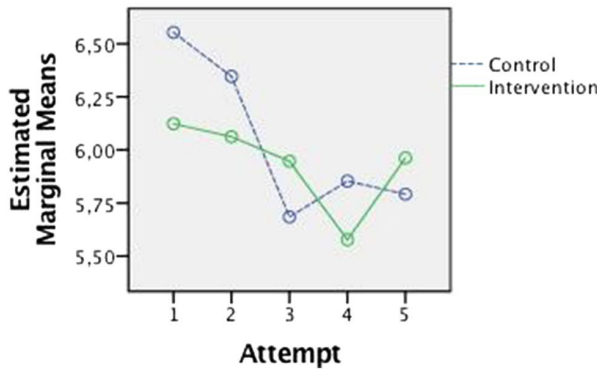

Figure 9 Needle targeting- master workspace range

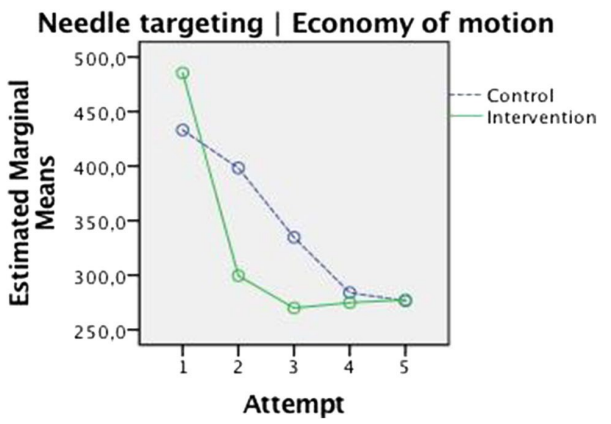

Figure $\mathbf{1 0}$ Needle targeting- economy of motion

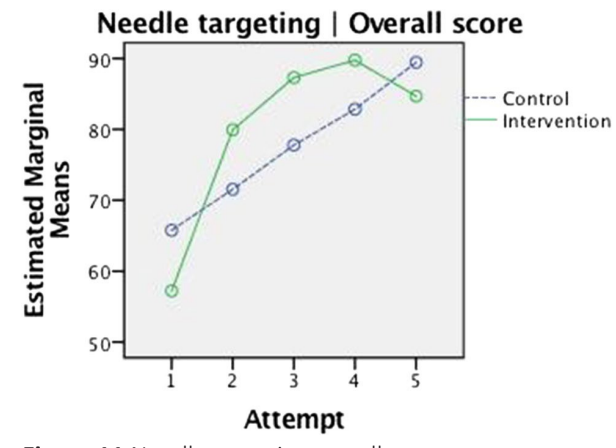

Figure 11 Needle targetig- overall score

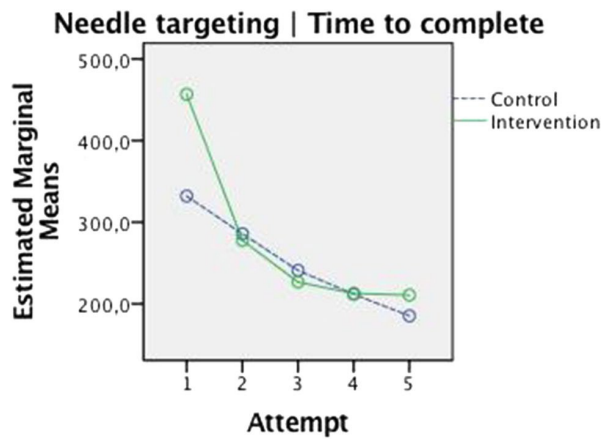

Figure 12 Needle targeting- time to complete

\section{Discussion}

This study was initiated after observing several expert surgeons in robotic-assisted procedures, where suboptimal body postures were frequently observed. The poor RULA scores in the control group demonstrates that an optimal posture is not guaranteed while using the console of da Vinci surgical system. With this study, the effect of a brief 
intervention on ergonomics was analysed. A simple instruction for novice users of the da Vinci surgical system simulator leads to a dramatic increase to posture.

RULA scores in both exercises highly improved after instructions. The right and left body half are both subject to less physical strain. The angles of all body joints calculated by RULA improve significantly after instructions on ergonomic position. Despite analysis of the RULA score sheets by two independent observers, optimal interpretation and $100 \%$ accuracy can never be guaranteed. With these data, however, it can be stated that the instructions lead to a better posture in the console.

The ergonomic instructions also result in a significantly smaller MWR in the scaling exercise, with fewer unnecessary movements. This is a promising result. Reproduction in the needling exercise (where less clutching is warranted) was not seen. Most likely, with these two different exercises, other aspects of ergonomic performance are investigated. When time needed to complete the exercise is addressed, it should be noted that participants received additional and more complex instructions in the intervention group. This might result in more tasks in the same time frame, presumably leading to a longer time needed to complete the exercise. Detailed analysis of the learning curve in performance scores at the needle targeting exercise, a steeper learning curve for EOM and time to complete the exercise is observed. Once a participant knows how to use all the buttons and clutches in a correct manner, the performance improves quickly. This interesting finding is not reported in the control group. Analysis shows that the overall performance score was not affected in the intervention group, even though participants received additional and more complex instructions.

With regard to mental and physical demand scores, no significant differences could be found. Several characteristics of this research project should be taken into account. The participants were all young and in excellent physical shape. In total, they spent only little more than 1 hour in the console. Longer operating times might affect the results of the questionnaires. Muscle strain during weeks or months is more likely to cause physical complaints than a single task in a short period of time.

This study builds on earlier research, bringing theory into (simulated) practice [1, 4, 9]. As demonstrated by Yang et al., armrest pressure can be used as a predictor for ergonomic posture [4]. Using arm support as a basis for instructions on a correct ergonomic use of the console, participants displayed excellent posture. Robot-assisted surgery offers opportunities to deal with the high incidence of musculoskeletal problems in surgeons performing laparoscopic surgery [10-14]. The console offers unique features to improve the ergonomic situation for surgeons. However, until recently, the need for ergonomic optimization has not received much attention. With a brief and simple instruction, combined with feedback on body posture during early training, ergonomics can be improved. Additional reduction of musculoskeletal strain for surgeons will be of major importance, now and in the future. Applying robotic systems in a safe and ergonomic correct manner is an important issue that can benefit many surgeons. 


\section{Conclusions}

Implementing a brief instruction about correct ergonomic position and console usage, combined with a short coaching session leads to optimal ergonomic outcomes. Several suggestions for future training regimens in robot-assisted surgery are confirmed and validated in this research project. It is important to verify optimal console settings and to optimize chair height. The armrest should be considered as ergonomics' best friend. Finally, individualized ergonomic instructions should include extensive coaching regarding clutch usage in training programs.

Acknowledgements Many thanks to the residents and interns at the Department of Surgery for their cooperation in this research project. 


\section{References}

1. Broeders IAMJ (2014) Robotics: the next step?. Best Pract Res Clin Gastroenterol 28(1):225-232

2. Raza SJ, Froghi S, Chowriappa A, Ahmed K, Field E, Stegemann AP et al (2014) Construct validation of the key components of fundamental skills of robotic surgery (FSRS) curriculum: a multi- institution prospective study. J Surg Educ 71(3):316-324

3. Sealed Envelope Ltd. Create a blocked randomisation list [Internet]. 2016 [cited 2016 Apr 5]. https://www.sealedenvelope.com/ simple-randomiser/v1/lists

4. Yang K, Perez M, Perrenot C, Hubert N, Felblinger J, Hubert J (2016) A new system for evaluation of armrest use in robotic surgery and validation of a new ergonomic concept: armrest load. Int J Med Robot Comput Assist Surg 12:604-612

5. Mcatamney L, Corlett EN (1993) RULA: a survey method for the investigation of world-related upper limb disorders. Appl Ergon 24(2):91-99

6. Corlett EN, Bishop RP (1976) A technique for assessing postural discomfort. Ergonomics 19(2):175-182

7. Hart SG (2006) NASA-task load index (NASA-TLX); 20 years later. Hum Factors Ergon Soc Annu Meet 50:904-908

8. Rubio S, Diaz E, Martin J, Puente J (2004) Evaluation of subjective mental workload: a comparison of SWAT, NASA-TLX, and workload profile methods. Appl Psychol 53:61-86

9. Van Der Schatte Olivier RH, Van 't Hullenaar CDP, Ruurda JP, Broeders IAMJ (2009) Ergonomics, user comfort, and performance in standard and robot-assisted laparoscopic surgery. Surg Endosc 23(6):13651371

10. Mohammad Mirbod S, Yoshida H, Miyamoto K, Miyashita K, Inaba R, Iwata H (1995) Subjective complaints in orthopaedists and general surgeons. Int Arch Occup Environ Health 67(3):179-186

11. Sari V, Nieboer TE, Vierhout ME, Stegeman DF, Kluivers KB (2010) The operation room as a hostile environment for surgeons: physical complaints during and after laparoscopy. Minim Invasive Ther Allied Technol 19(2):105-109

12. Auerbach JD, Weidner ZD, Milby AH, Diab M, Lonner BS (2011) Musculoskeletal disorders among spine surgeons: results of a survey of the Scoliosis Research Society membership. Spine 36(26):E1715-1721

13. Dalager T, Søgaard K, Jensen PT (2016) Musculoskeletal pain among surgeons performing minimally invasive surgery: a systematic review. Surg Endosc 31:516-526

14. Tzeng Y-S, Chen S-G, Chen T-M (2012) Herniation of the cervical disk in plastic surgeons. Ann Plast Surg 69(6) 


\section{Appendix 1. Instructions on usage of the Da Vinci simulator}

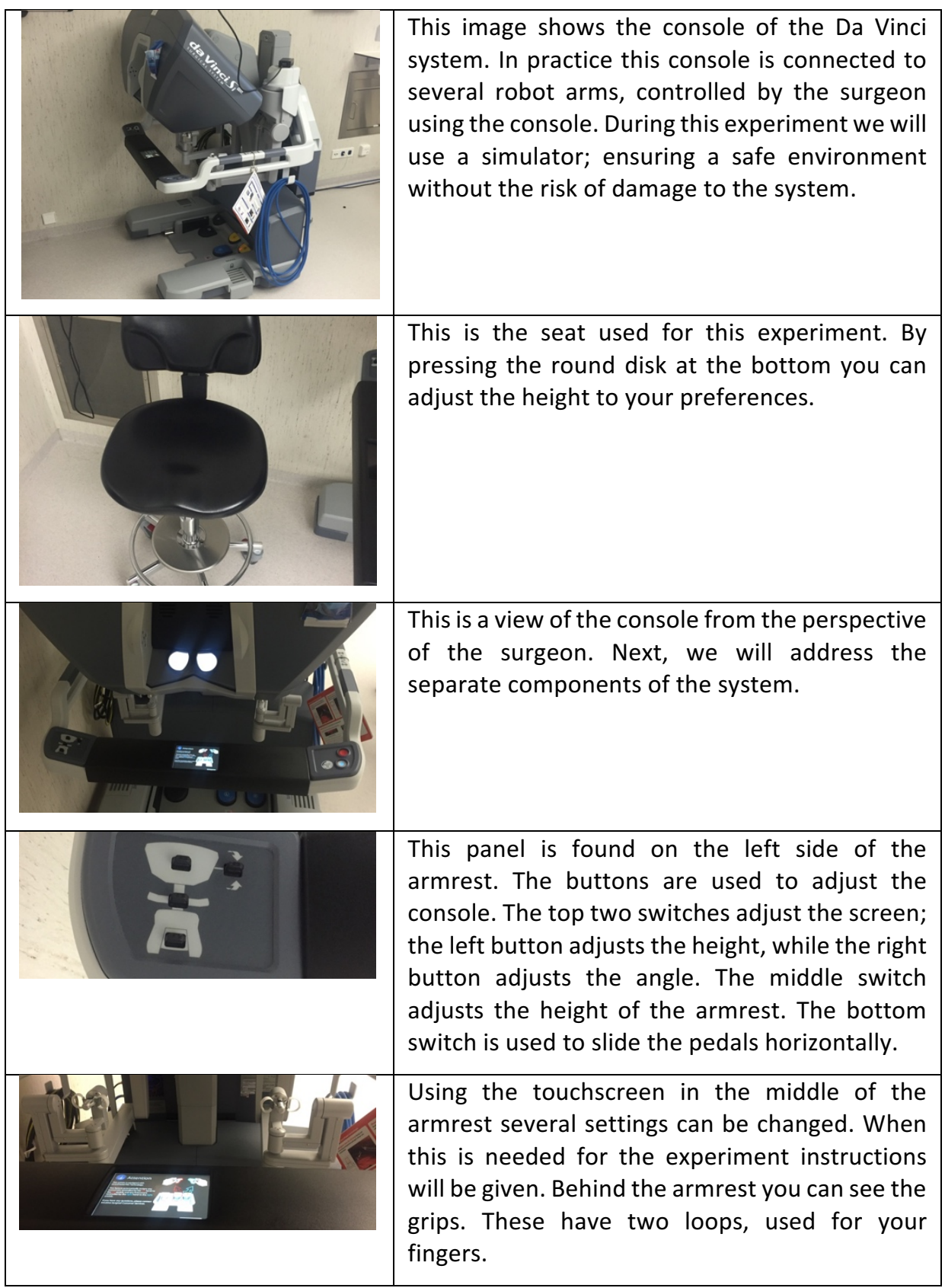




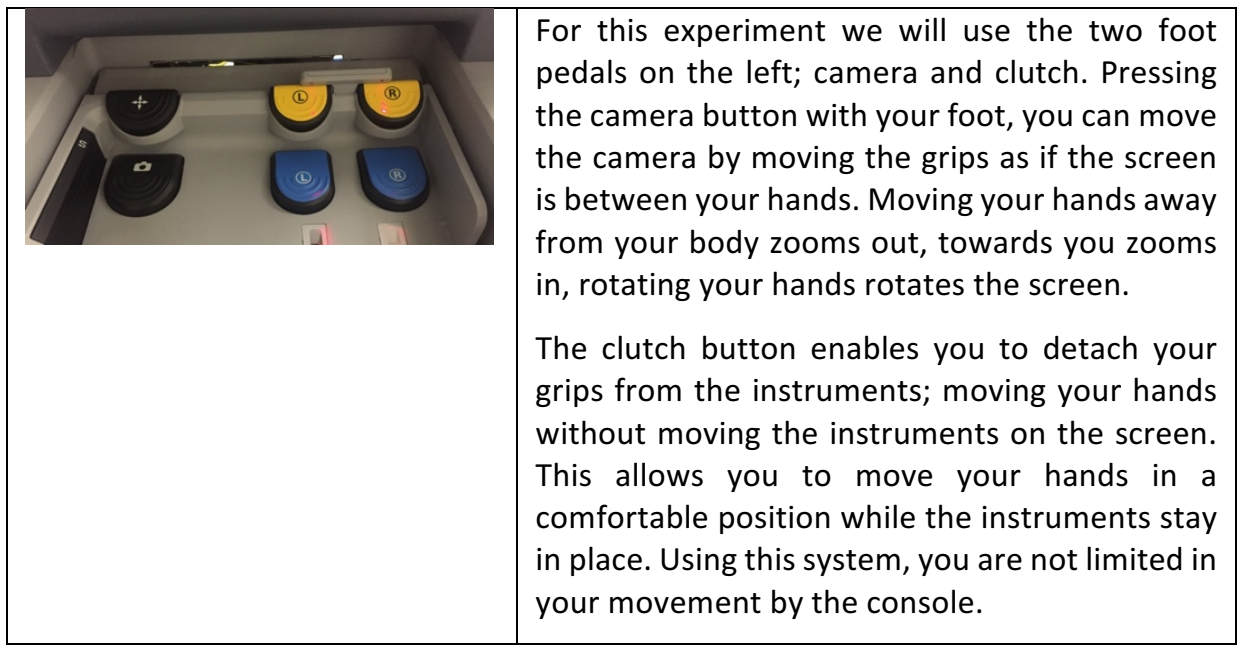




\section{Appendix 2. Additional instructions on ergonomics}

\section{Adjusting the seat}

Feet flat on the floor with your legs only just supported by the seat, keep a straight back.

\section{Setting the console}

Pedals in comfortable reach of your feet, while leaving room to rest your feet. Adjust the armrest height so your arms are supported and shoulders are just relaxed.

Adjust the console height and angle; take care to set the screen high enough to keep a straight back and prevent your neck from bending forward.

\section{Clutch usage}

As noted earlier, the clutch is a tool that is used to allows you to move your hands without moving the instruments. Many people use the clutch only when they are physically limited by the console; when the grips are touching the console. This leads to uncomfortable positions for the user of the system. The clutch allows you to maintain a comfortable position while you perform complex movements with the instruments. As a rule of thumb, use the clutch when you feel like moving your arms from the armrest. When you engage the clutch, move your hands back into the neutral starting position. In the end, this allows you to work faster, more comfortable and more precise. 




\section{Chapter}

\section{Ergonomic assessment of the first assistant during robot-assisted surgery}

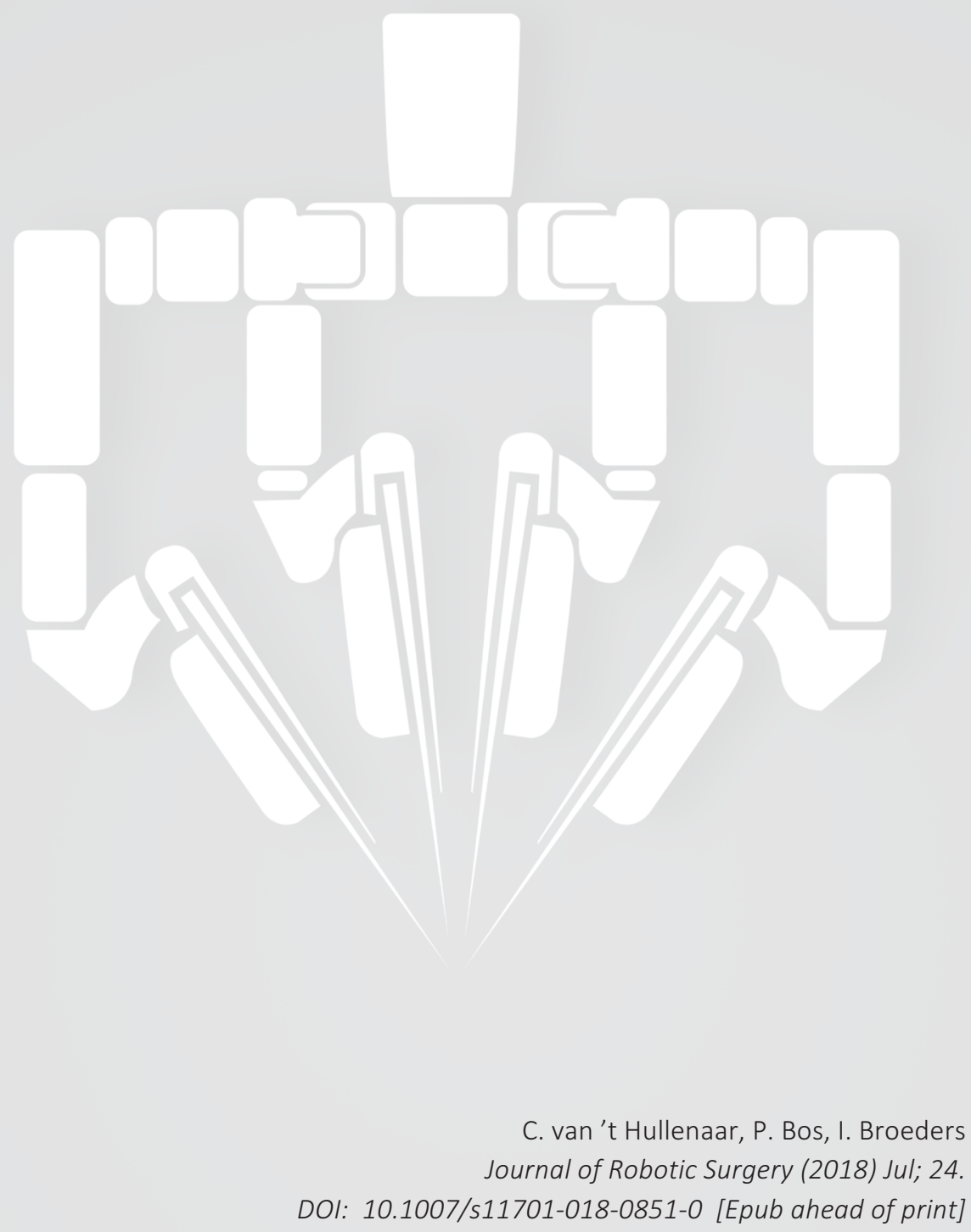




\begin{abstract}
Background The use of the da Vinci robot in minimal invasive surgery comes with numerous advantages. Recent papers describe improvements in the ergonomic environment and benefits for the surgeon's posture in the console. Ergonomics for first assistants or scrub nurses at the OR-table during robot-assisted procedures however, have gained minor attention. The aim of this study is to analyse the ergonomic environment specifically for first assistants during robot-assisted surgery.

Methods Three techniques were used to evaluate body posture and ergonomics during three discriminated actions. First of all, a questionnaire was conducted. Secondly, sagittal and dorsal photographs of all first assistants were shot. From these photographs, joint angles of the trunk, neck, shoulder, elbow, pelvic girdle and spine were calculated and rapid upper limb assessment (RULA) scores were determined. In addition, intra-observer variability was assessed to determine the robustness of the results. Thirdly, the number of obstructions during the surgery was registered by an observer present at the operating theatre.
\end{abstract}

Results The questionnaires displayed that seventy-three percent of the first assistants were in uncomfortable working positions for longer periods of time. Twenty percent of the participants even report pain or visible bruising due to hinder of the robot arm. Furthermore, an average of 2.8 obstructions per surgical procedure were registered, mainly affecting the lower arm (60 percent). The photographs demonstrated that all joint angles, except for the elbow joint, are potentially harmful when assisting during robotassisted surgery. RULA scores revealed high risk ergonomic risk scores for all measured actions. Tissue traction was recognized as the action with the highest physical workload.

Conclusion During robot-assisted surgery, first assistants experience non-ergonomic trunk, neck and shoulder angles. These recordings are supported by posture analysis. Tissue traction is reported as the most intensive action by the nurses. Tacking however can lead to the most unfavourable RULA score. The surgeon's awareness of the position of the robot arms could reduce the number of obstructive moments for the first assistant. Lowering the number of instrument replacements is plausible to lead to better ergonomic postures for first assistant. 


\section{Introduction}

The number of robot-assisted surgical procedures is increasing by more than $15 \%$ annually. In 2017, approximately 877.000 procedures were performed worldwide with the da Vinci systems. [13] Improvement of the ergonomic environment and reduction of physical strain for the surgeon during minimal invasive surgical procedures is an particular advantage of working with de da Vinci system. [1-3] Several studies demonstrated ergonomic discomfort for surgeons while performing laparoscopic procedures.[4-7, 14, 15] This can lead to musculoskeletal symptoms such as pain, fatigue and stiffness. The da Vinci robot systems tackle numerous ergonomic disadvantages. Sitting in a console with an armrest leads to more comfort and by using the instrument clutch feature, large movements in the shoulder, elbow and wrists can be avoided.[8] Although most research focusses on ergonomic advantages for the surgeon, the da Vinci system might negatively influence body posture of first assistants. Since first assistants play a crucial role in all robot-assisted procedures, their ergonomic situation is of major importance. When performing surgery from the console, the surgeon is not always aware of the position of the robot arms. This can lead to unfavourable ergonomics and potential hazardous situations for the first assistant. Additionally, the robot arms cause discomfort when a change of instruments or assistance during a procedure is warranted. Therefore the goal of this study is to analyse ergonomics for the first assistant during robot-assisted procedures.

\section{Methods}

\section{Participants \& Questionnaires}

Eleven first assistants of the Meander Medical Center (Amersfoort, the Netherlands) participated in this study. All participants were experienced nurses; all assisted in more than 30 robot-assisted cases. All first assistants filled out a five minute questionnaire regarding the ergonomic environment. Additionally, the validated Dutch Musculoskeletal Questionnaire, which analyses musculoskeletal symptoms due to the workload, was also filled out by all participants.[9]

\section{Ergonomic evaluation}

\section{Joint angles}

To evaluate the ergonomic posture of the first assistant during robot-assisted surgery, multiple sagittal and dorsal plane photographs were taken during three common actions: 1 ) tissue traction, 2) tissue tacking and 3 ) instrument replacement. Anatomical landmarks were used to calculate the angles from the neck $(\Delta N)$, trunk $(\Delta T)$, shoulder $(\Delta S)$ and elbow $(\Delta E)$ in the sagittal plane during these actions. Angles of the neck $(\Delta N)$, trunk $(\Delta T)$, shoulder girdle $(\Delta \mathrm{dS})$, pelvic belt $(\Delta \mathrm{dP})$ and torsion of the spine ( $\Delta \mathrm{dTS})$ were analysed in the dorsal plane photographs. 
Optimal joint angles were determined, based on the RULA score. This includes angles of $10^{\circ}$ for the neck $(\Delta N)$ and the trunk $(\Delta T)$. Shoulder angles $(\Delta S)$ are ergonomic up to $20^{\circ}$ anteflexion or abduction $(\Delta \mathrm{dS})$. For elbow angles $(\Delta \mathrm{E})$ this range is between $60^{\circ}$ and $100^{\circ}$ flexion. $[8,10]$ For the remaining angles, dorsal photographs were used. An angle higher than $5^{\circ}$ outside the range was considered potentially harmful to the optimal ergonomic situation.

All joint angles are calculated using Matlab 2013b (The MathWorks, Natick, MA, USA), as visualized in figure 1 . The trunk angle was calculated by comparing a vertical line with a straight line through the marked points. This 'backfit' was measured using the standard least squares method. The angle between this 'backfit' line and the line between vertebrae $\mathrm{C} 1$ and $\mathrm{C} 7$ represents the neck angle. The shoulder angle was calculated by drawing a line between the olecranon and the acromion. Torsion of the spine was measured by $\Delta T S=\frac{\Delta S}{\Delta P}$.

Intra-subject variability was determined by analysing two participants ten consecutive times and calculating the standard deviation from these results.

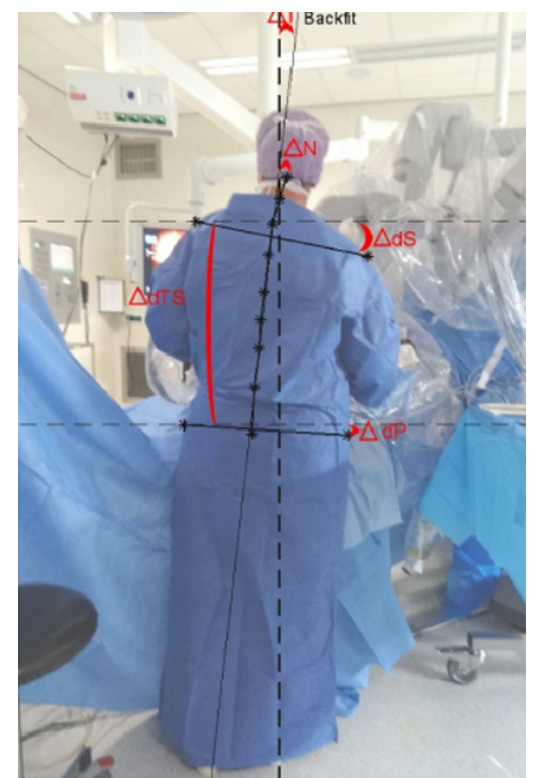

(a) Dorsal plane

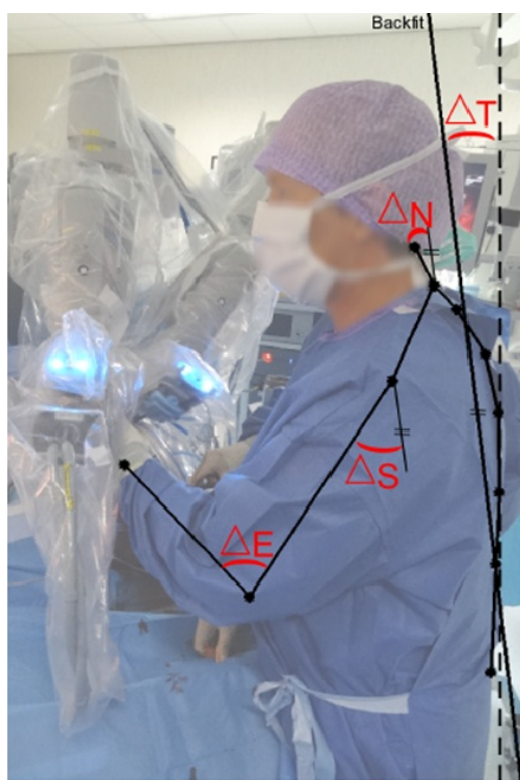

(b) Sagittal plane

Figure 1: Representation of the angles calculated from the different planes. ( $\Delta N)$ Neck; $(\Delta T)$ Trunk; $(\Delta S)$ Shoulder; $(\Delta \mathrm{E})$ Elbow; $(\Delta \mathrm{dS})$ Shoulder girdle; $(\Delta \mathrm{dP})$ Pelvic belt; $(\Delta \mathrm{dTS})$ Torsion of the spine angles.

\section{Rapid upper limb assessment}

The rapid upper limb assessment (RULA) is a validated and reliable tool to evaluate workrelated upper limb ergonomics.[10] This RULA score, ranging from one to seven, determines the severity of the ergonomic exceedances. A low score means optimal 
ergonomics, a high score represents a serious health thread. Due to the often invisible wrist position on the photographs caused by the arms of the robot, it was not possible to determine a separate wrist score. Assuming the wrist joint is often more than $15^{\circ}$ bended, this results in a RULA score of three. The mean RULA score per participant in every action category was determined by calculating the mean angle in all photographs of that participant.

\section{Time frames}

To validate the outcome parameters of the questionnaire, time frames of each activity of the first assistant were timed in a score sheet. During tacking, the time frame was defined as the time between the first and the last tacking session. It is important to realize that the length of time frames influences the RULA score. Subsequently, the number of obstructions caused by the robot arms was registered with a score sheet. Obstructions were defined as unintentional contact of the robot arms with the arms, trunk or head of the first assistant.

\section{Results}

In total, thirteen procedures with eleven first assistants were analysed. Three specialities were included this research project: Urology, Gynaecology and General Surgery. An overview of all procedures is displayed in table 1. Also, the various RULA scores with standard deviations of the different tasks are displayed here.

\section{Questionnaires}

The mean exposure of the participants with robot-assisted procedures was 14 hours weekly. The mean work experience with robot-assisted surgery is 3.0 years [range 2.0 4.0 years].

Seventy-three percent of the first assistants reported working in a bended, stooped or twisted posture for longer periods is common practice. This can lead to musculoskeletal strain on trunk, neck and wrist. Short repetitive movements are common for the neck (82\%), the trunk (73\%) and the wrist (73\%). In addition, participants mentioned several work-related activities with their arms above shoulder level. Uncomfortable postures, such as reaching out for the robot arm and working in the same position for a longer period of time, were reported by all participants. Moreover, a limited work-space was noticed due to the robot arms. This can result in static postures, obstruction and hindrance. The questionnaires revealed that twenty percent of the participants reported pain or visible bruising due to interference with the robot arm. Obstructions, reported when accidental contact of the robot arm with the first assistants was recorded, occurred on average 2.8 times [range $0-8$ ] per surgical procedure. This was mainly affecting the lower arm (60\%). Position of the monitor plays a vital role in minimal invasive ergonomics. However, monitor position preferences display a wide 
variation. The monitor was mostly positioned on the same side as the first assistants, between the patient and the instrument table (45\%).

Table 1: RULA scores of all procedures (mean \pm standard deviation)

\begin{tabular}{|c|c|c|c|c|c|c|}
\hline \multirow[b]{2}{*}{ No. } & \multirow[b]{2}{*}{ Surgery } & \multirow[b]{2}{*}{ Specialism } & \multirow[b]{2}{*}{$\begin{array}{l}\text { Assis- } \\
\text { tant }\end{array}$} & \multicolumn{3}{|c|}{ RULA scores } \\
\hline & & & & $\begin{array}{l}\text { Tissue } \\
\text { traction }\end{array}$ & Tissue tacking & $\begin{array}{l}\text { Instrument } \\
\text { replacement }\end{array}$ \\
\hline 1 & Redo fundoplication & General surgery & $\mathrm{MB}$ & 3.0 & NA & NA \\
\hline 2 & Redo fundoplication & General surgery & PD & $3.5 \pm 0.7$ & NA & NA \\
\hline 3 & Abdominoperineal resection & General surgery & MM & $5.3 \pm 0.5$ & 3.0 & $5.7 \pm 1.5$ \\
\hline 4 & Prostatectomy & Urology & NM & $3.7 \pm 0.6$ & NA & NA \\
\hline 5 & Sacrocolporectopexy & Gynaecology & NM & $4.0 \pm 1.4$ & NA & NA \\
\hline 6 & $\begin{array}{l}\text { Rectovaginopexy + } \\
\text { Sacrocolporectopexy }\end{array}$ & $\begin{array}{l}\text { General surgery + } \\
\text { Gynaecology }\end{array}$ & $M V$ & $5.3 \pm 1.0$ & $4.5 \pm 2.1$ & NA \\
\hline 7 & Rectovaginopexy & General surgery & PD & $3.7 \pm 1.2$ & 4.0 & 5.0 \\
\hline 8 & Pyleumplasty & Urology & MV & $5.0 \pm 1.4$ & 6.0 & NA \\
\hline 9 & Redo fundoplication & General surgery & RS & $3.3 \pm 0.6$ & 6.0 & NA \\
\hline 10 & Prostatectomy & Urology & EvD & $3.3 \pm 0.5$ & $3.5 \pm 0.7$ & 7.0 \\
\hline 11 & Sacrocolporectopexy & Gynaecology & EvZ & $6.0 \pm 1.0$ & $5.0 \pm 1.4$ & 7.0 \\
\hline 12 & Sacrocolporectopexy & Gynaecology & $\mathrm{RD}$ & $5.0 \pm 1.0$ & $5.5 \pm 0.7$ & $5.0 \pm 1.7$ \\
\hline 13 & Sacrocolporectopexy & Gynaecology & AK & $6.0 \pm 0.0$ & $3.8 \pm 1.0$ & $6.5 \pm 0.7$ \\
\hline 14 & Rectopexy & General surgery & RS & $3.5 \pm 0.7$ & 5.0 & $4.5 \pm 0.7$ \\
\hline
\end{tabular}

If only one photograph of a certain action is available, there is no standard deviation mentioned NA: not applicable

\section{Ergonomic evaluation}

\section{Joint angles}

The mean joint angles of all participants for each defined action are displayed in figure 2 . The green shaded area represents the preferable joint angles defined by RULA. As can be seen, all joint angles - except for the elbow joint - are potentially harmful. In addition, the neck joint is on the edge of the danger zone during tissue tacking and traction. In both planes (dorsal and sagittal), instrument replacement was determined as the most harmful action, followed by tacking and tissue traction. This was recorded in all joints, except the elbow joint. The intra-subject variability in both planes is below $5^{\circ}$ for the neck angles and below $2^{\circ}$ for the other joint angles. 


\section{Sagittal}

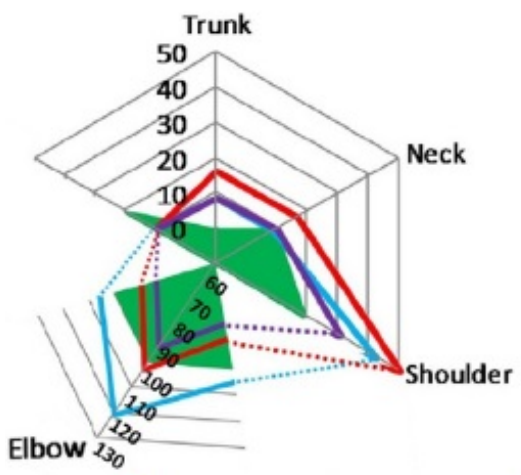

Instrumental change

\section{Dorsal}

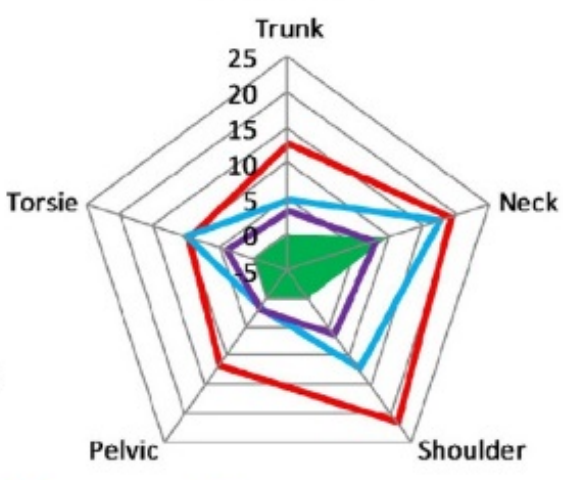

Tacking Draction

Figure 2: Spider plot of the mean angles of the measured joints in the dorsal and sagittal plane, where the green area is defined as not harmful.

\section{RULA}

The mean time frames of the actions are summarized in table 3. This influences the RULA score. For tissue traction and tissue tacking, an additional point in the RULA score is calculated for static posture, because these actions take longer than 60 seconds. The mean RULA score per first assistant is illustrated in table 1. The average RULA scores of subspecialties are summarized in table 2. These tables demonstrate that the highest RULA score was found during tacking. Gynaecology can be seen as the specialism with the most uncomfortable ergonomic body postures for the first assistant.

Table 2: RULA scores (mean and standard deviation) of all first assistants during specific tasks in all fourteen surgical procedures, specified in different specialisms.

\begin{tabular}{lllll}
\hline Specialism & Tissue traction & Tissue tacking & Instrument replacement & Overall \\
\hline General & $4.3 \pm 1.1$ & $5.8 \pm 1.0$ & $4.6 \pm 1.0$ & 4.9 \\
General surgery & $3.7 \pm 0.8$ & $5.1 \pm 0.6$ & $4.5 \pm 1.3$ & 4.4 \\
Gynaecology & $5.3 \pm 0.8$ & $6.2 \pm 1.0$ & $4.7 \pm 0.7$ & 5.4 \\
Urology & $4.0 \pm 0.9$ & $7.0 \pm 0.0$ & $4.8 \pm 1.8$ & 5.3 \\
Overall & 4.3 & 6.0 & 4.7 & \\
\hline
\end{tabular}


Table 3: RULA scores (mean and standard deviation) of all first assistants during specific tasks in all fourteen surgical procedures, specified in different specialisms.

\begin{tabular}{llcl}
\hline Specialism & Tissue traction & Tacking & $\begin{array}{l}\text { Instrument } \\
\text { replacement }\end{array}$ \\
\hline General Surgery & 177 & 142 & 38 \\
Gynaecology & 234 & 81 & 58 \\
Urology & 212 & 134 & 65 \\
Overall & 207 & 119 & 54 \\
\hline
\end{tabular}

\section{Discussion}

To date, no previous study was published describing the ergonomic environment for the first assistant during robot-assisted surgery. This issue was recognized as a serious point of concern at the European Association of Endoscopic Surgery (EAES) consensus meeting on robot-assisted surgery.[16]

Three techniques to evaluate ergonomics were applied in this study: a questionnaire, photographs in the operation room and application of a RULA score sheet.

When only taking into account the joint angles derived from the photographs, instrument replacement is defined as the least ergonomic action. The RULA score however, determines tacking as the least ergonomic action. This discrepancy is caused by factors like static postures and repetitive movement that are being included in the RULA score, but cannot be measured in the photographs. Moreover, tackers are placed deep in the pelvic space and this can also result in more physical strain and uncomfortable joint angles. The fact that Gynaecology is the specialism where most ergonomic uncomfortable positions are recorded, is probably caused by extensive tissue traction and frequent instrument replacements, as well as manipulation of the uterus simultaneously with other tasks.

In the questionnaires, tissue traction was addressed as the action with the highest physical intensity level. This is caused by the static posture that is essential during tissue traction. Although joint angles are often not extreme during tissue traction, the intensity of the action is caused by the time needed to perform it. In contrast, tacking and instrumental replacement are brief actions. Thereby such actions require the full attention and concentration of the first assistant; awareness for non-ergonomic joint angles might be lower at such moments.

The joint angles during instrument replacement display extreme values. This is based on the fact that replacement of the instruments of the third robot arm (the furthest arm) demands extensive bending and reaching out from the first assistant. This causes extreme joints angles. Less often replacing instruments of the third arm could therefore result in a reduction of physical strain for the first assistant. Another solution for the first assistant could be to change position. For instance, when standing on the other side of the patient, instrument replacements at the third robot arm can be less stressful and harmful. 
To overcome work-related musculoskeletal disorders, optimal hardware settings are required. Monitor position is vital to reduce rotation and twisting of the neck and back.[11,12] Due to the robotic chart in the operation area, an optimal monitor position (monitor in line with the viewer) cannot always be achieved. Results from the questionnaire show wide inter- and intra-subject variability in the use of monitor location. The preferred monitor position is dependent on the specialism, the type of surgery and the type of action. Perioperative placement of more monitors at optimal view angles might lead to more ergonomic body postures.

Operation and instrument tables are adjustable in height. However, the operation table is limited in its movement during robot-assisted surgery. The table cannot always be put at an optimum height and several factors disturb an optimal operation table height. For instance, the condition of the patient can limit the amount of (reversed) Trendelenburg position. The preferences of the surgeon and the process of docking of the da Vinci patient-side cart also determine the final height of the operation table. The first assistant therefore frequently has to compensate a suboptimal table position by working with a larger elbow angle. It is of clear importance that evaluating table height during every procedure can reduce serious strain during surgical procedures.

The da Vinci Si Surgical System operates with three or four robot arms that pass trocar portals. At the start of the procedure, additional trocar portals are placed. Such a remaining trocar port is used by first assistants to assist during procedures. When this trocar port is positioned too close to the trocar portals used by the robot arms, this will result in a limited and narrow working space for the first assistant. With regard to the elbow and wrist, this means major twisting and bending. Combined with the continuous movements of the robot arms navigated by the surgeon, serious hinder and discomfort are experienced by the first assistant. Good communication between the surgeon and first assistant is likely to reduce this discomfort.

Our questionnaire recorded a mean hindrance of at least one incident per surgical procedure. However, our objective score sheet measurement determined an average obstruction number of three times per surgery. This difference can be explained by the fact that some level of hindrance is 'accepted' by the first assistants and is not even addressed in a questionnaire.

This study is based on photographs of the first assistant. According to our intraobserver variability, this study method is reproductive and reliable. However, sometimes it can be difficult to locate all anatomical landmarks when the nurse is wearing a sterile jacket. This might influence accuracy of the measurements.

\section{Conclusion and points of advice}

During robot-assisted surgery, the first assistant experiences non-ergonomic trunk, neck, shoulder and wrist angles. Tissue traction is reported as the most intensive action by the participants. The highest RULA score was found during tacking. The outcome of these RULA scores display that all actions need further ergonomic investigation. 
To reduce discomfort and optimize ergonomics, several points of advice can be formulated. First of all the first assistant needs to be aware of optimal monitor and table settings. They have to take care of a comfortable position, with an acceptable reach distance to instruments and trocar portals.

Secondly, to prevent extreme joint angles for the first assistant, the most remote trocar portal must be used as little as possible, especially for instrument replacements. In conjunction, to reduce the strain for the nurse, the surgeon can place an instrument at this portal just before entering the console.

Awareness of the trocar portal positions is vital to optimize ergonomics. Careful consideration of placement of the assistant trocar portals will result in more freedom of movement for the first assistant. A distance of $8 \mathrm{~cm}$ (>3inches) at minimum is advised between trocar portals. Surgeons need to be aware of the fulcrum effect: large external movements of the robot arms can be reduced by placing trocars further from the target organ. A smaller range of motion outside the body is generated by placing a larger part of the instrument inside the body.

Besides that, surgeons need to be aware of the relation between the external movements of the robot arms. Calm and smooth movements in the console lead to slower movements of the robot arms. This can reduce hindrance for the first assistant because there is more time to anticipate.

Intensive communication between the surgeon and first assistant is essential. The team has to take care that visual contact between the surgeon and the operation team is possible from the console. Verbal communication can be optimized by correct speaker settings. Clear instructions regarding an expected large range of motion, a sudden change in camera position or placement of instruments outside the optical field are crucial. Lastly, when briefing for a procedure, explicit communication with your team on ergonomics is a key part of robot-assisted surgery. Check the patient position, check the hardware and software settings and make your team aware for the movement of the robot arms. 


\section{References}

1. Lanfranco AR, Castellanos AE, Desai JP, and Meyers WC (2003). Robotic Surgery: A current perspective. Annals of surgery 239(1):14-21

2. Chopra S, Srivastava A, and Tewari A (2012). Robotic radical prostatectomy: The new gold standard. Arab Journal of Urology 10(1):23-31

3. Lawson EH, Curet MJ, Sanchez BR, Schuster R, and Berguer R (2007). Postural ergonomics during robotic and laparoscopic gastric bypass surgery: a pilot project. Journal of Robotic Surgery 1(1):61-67

4. Broeders IAMJ (2014). Robotics: The next step? Best practice \& research. Clinical gastroenterology 28(1):225-32

5. Sari V, Nieboer TE, Vierhout ME, Stegeman DF, and Kluivers KB (2010). The operation room as a hostile environment for surgeons: physical complaints during and after laparoscopy. Minimally invasive therapy 19(2):105-9

6. Van Veelen MA, Nederlof EAL, Goossens RHM, Schot CJ and Jakimowicz JJ (2003). Ergonomic problems encountered by the medical team related to products used for minimally invasive surgery. Surgical endoscopy 17(7):1077-81

7. Zihni AM, Ohu I, Cavallo JA, Cho S, and Awad MM(2014). Ergonomic analysis of robot-assisted and traditional laparoscopic procedures. Surgical endoscopy DOI 10.1007/s00464-014-3604-9

8. Van 't Hullenaar CDP, Hermans, B, and Broeders, IAMJ (2017). Ergonomic assessment of the da Vinci console in robot-assisted surgery. Innovative Surgical Sciences. 2. 10.1515/iss-2017-0007.

9. Hildebrandt VH, Bongers PM, van Dijk FJ, Kemper HC and Dul J (2001). Dutch Musculoskeletal Questionnaire: description and basic qualities. Ergonomics 44(12):1038-55

10. Mcatamney L and Corlett EN (1993). RULA : a survey method for the investigation of work-related upper limb disorders. Applied ergonomics 24(2):91-99

11. Tjiam OM, Goossens RJ, Schout BM, Koldewijn EL, Hendrikx AJ, Muijtjens AM, Scherpbier AJ and Witjes JA (2014). Ergonomics in endourology and laparoscopy: an overview of musculoskeletal problems in urology. Journal of endourology Endourological Society 28(5):605-11

12. ihallaly GEL and Cuschieri A (2006). Optimum view distance for laparoscopic surgery. Surgical endoscopy 20:1879-82

13. Preliminary fourth quarter 2017 revenue Intuitive Surgical (2018). http://phx.corporate-ir.net/phoenix. zhtml?c=122359\&p=irol-newsArticle\&ID=2325993

14. Alleblas CCJ, de Man AM, van den Haak L, Vierhout ME, Jansen FW, Nieboer TE. Prevalence of Musculoskeletal Disorders Among Surgeons Performing Minimally Invasive Surgery: A Systematic Review. Ann Surg. 2017 Dec;266(6):905-920. doi: 10.1097/SLA.0000000000002223. Review.

15. Alleblas CC, Velthuis S, Nieboer TE, Sietses C, Stegeman DF. The Physical Workload of Surgeons: A Comparison of SILS and Conventional Laparoscopy. Surg Innov. 2015 Aug;22(4):376-81. doi: 10.1177/1553350615577480. Epub 2015 Mar 22. PMID: 25801191

16. Szold A, Bergamaschi R, Broeders I, Dankelman J, Forgione A, Lang $\varnothing$ T, Melzer A, Mintz Y, Morales-Conde S, Rhodes M, Satava R, Tang CN, Vilallonga R. European Association of Endoscopic Surgeons (EAES) consensus statement on the use of robotics in general surgery. Surg Endosc. 2015 Feb;29(2):253-88. doi: 10.1007/s00464-014-3916-9. 



\section{Chapter}

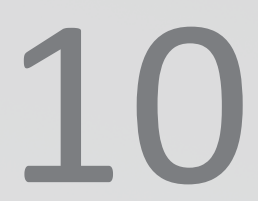

\section{Ergonomics in hand-held and robot-assisted camera control: a randomised controlled trial}

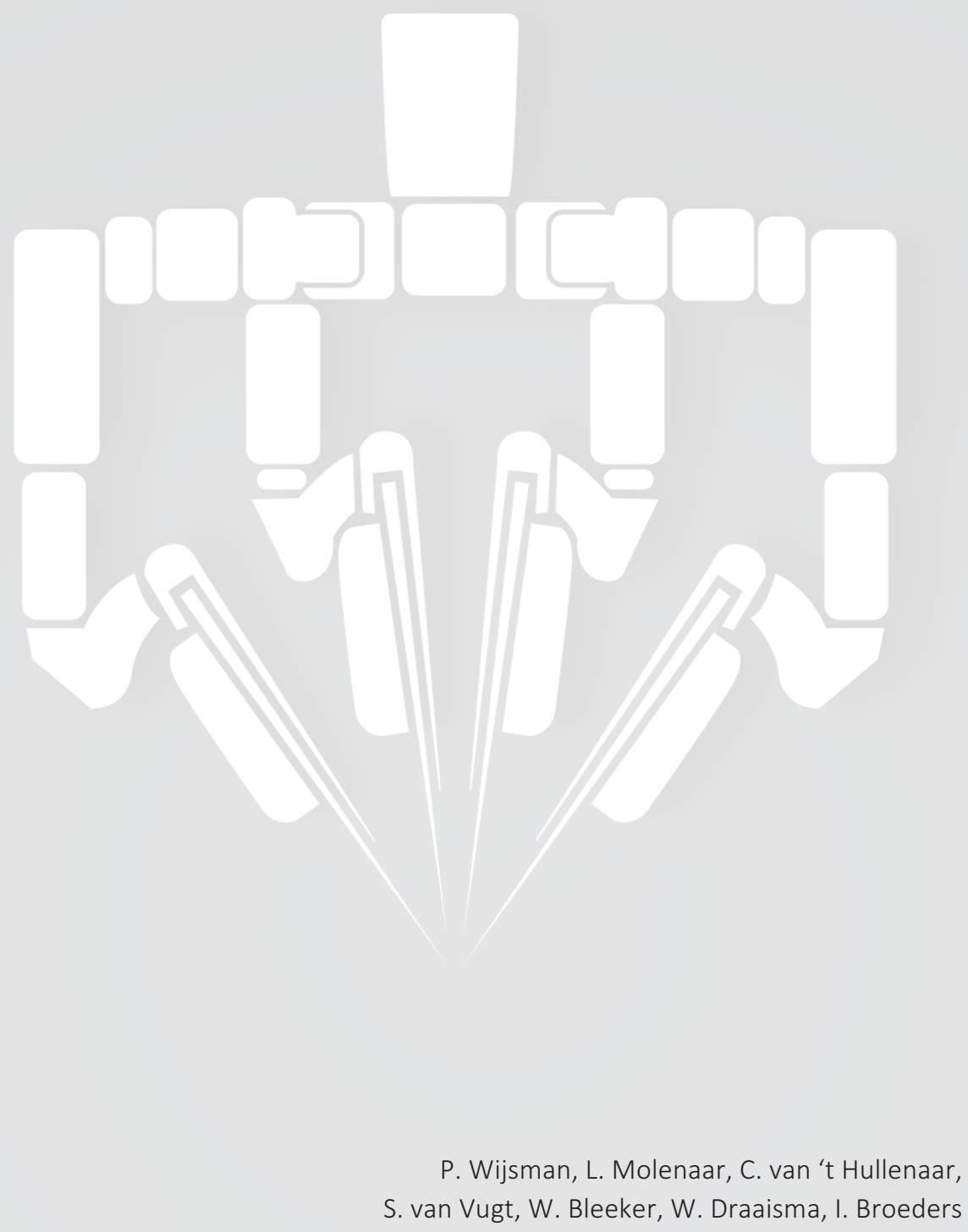




\section{Abstract}

Background: Laparoscopic surgery potentially increases physical burden to operating theatre staff and can cause physical discomforts. This study aims to evaluate whether a robotic camera holder (AutoLap ${ }^{\mathrm{TM}}$ system) can improve ergonomics for the surgeon and the camera-assistant during laparoscopic procedures.

Methods: A total of thirty cases were included and randomised using stratified block randomisation (15 AutoLap ${ }^{\mathrm{TM}}, 15$ control). Five types of surgery were included: right hemicolectomy, fundoplication, sigmoid resection, rectopexy and low anterior resection. The postures of the surgeon and the assistant were photographed at pre-determined steps in the procedure. MATLAB was used to calculate the angles relevant to the RULA scores. Two investigators independently assessed the RULA scores. Also, three subjective questionnaires (SMEQ, NASA TLX and LED) were used to assess mental and physical discomfort.

Results: No differences in patient characteristics were observed. Sixteen fundoplications, seven right hemicolectomies, five sigmoid resections, one rectopexy and one low anterior resection were included. The mean RULA score of the surgeon was comparable in both groups, 2.58 (AutoLap ${ }^{\mathrm{TM}}$ ) versus 2.72 (control). The mean RULA score of the assistant was significantly different in both groups, with 2.55 in the AutoLap ${ }^{\mathrm{TM}}$ group versus 3.70 in the control group, $p=0.000$. The inter-observer variability (ICC) was excellent with 0.93 and 0.97 concordances. The questionnaires showed a significant difference in physical discomfort to the assistant. The LED score was 0.7 in the AutoLap ${ }^{\text {TM }}$ groups vs. 10.47 in the control group. For the SMEQ 28.8 vs. 53.3 was reported. For the NASA TLX, a significant reduction on all domains, except the mental domain was also established: mental (4.97 vs. 7.17 ), physical (4.10 vs. 9.00), temporal (2.8 vs. 5.47), performance (4.07 vs. 6.53 ), effort (4.93 vs. 8.13) and frustration (2.77 vs. 4.53 ), all in favour of the AutoLap ${ }^{\mathrm{TM}}$ system.

Conlusion: Robotic camera holders lead to improvements in the ergonomics and the posture of the first assistant. The ergonomics of the surgeon are not affected by the introduction of a robotic camera holder. Furthermore, the subjective work load is significantly reduced by the use of the AutoLap ${ }^{\mathrm{TM}}$ system. 


\section{Background}

Laparoscopic surgery can lead to an increased physical burden to operating room staff. Several studies demonstrate that physical discomfort is frequently reported by all members of the laparoscopic surgical team. [1-5] Surgeons, as well as their first assistants who steer the laparoscope, often work in unfavourable positions. The ergonomics of the first assistant are frequently compromised, yet show an optimal image for the surgeon. This is merely due to the first assistant standing aside from the central working axis, with this bringing about a torsion of the back and asymmetrically burdening the legs and shoulders. Recently, robotic camera holders were introduced into the field of minimal invasive surgery. This can potentially lead to improved ergonomics. [6]

The laparoscope is stabilized with the support of a robotic camera holder, relieving the first assistant of his duty of controlling the camera. This study saw the AutoLap ${ }^{\mathrm{TM}}$ system (Medical Surgery Technologies Ltd., Yokneam, Israel) deployed. This robotic arm can be controlled by the surgeon through the use of a sterile wireless joystick. This joystick has a ringed shape and is worn over the index finger. The AutoLap ${ }^{\mathrm{TM}}$ system is also equipped with active image analysis software. This software helps the surgeon to steer the camera towards a desired location. The tip of an instrument is identified using motion analysis software and the surgeon can point out any location and subsequently centre the camera with a single click on the joystick. This study looked at the role of a robotic camera holder in relation to the ergonomics of the surgical team.

\section{Methods}

This prospective multicentre randomized controlled trial analysed the ergonomics and posture of surgeons and their first assistants. Measurements were taken at two different hospitals, i.e. the Meander Medical Center, Amersfoort, and the Wilhelmina Hospital, Assen. Three surgeons and multiple first assistants participated in this trial. A sample size calculation was conducted prior to initiating this study. It was estimated that the use of a robotic camera holder would yield an improvement to the primary outcome (RULA score) by $10 \%$ of the total RULA score $(0.7$ improvement). Assuming equal variance, this resulted in a sample size of 30 cases.

A total of thirty cases were included and randomised using stratified block randomisation (15 AutoLap ${ }^{\mathrm{TM}}, 15$ control). Castor EDC software set random block sizes to reduce selection bias due to predictability of the treatment allocation. Five different type of procedures were included: laparoscopic right hemicolectomy, laparoscopic fundoplication, laparoscopic sigmoid resection, laparoscopic rectopexy and laparoscopic low anterior resection. Sagittal and dorsal postures of the surgeon and the first assistant were recorded by taking photographs of predetermined steps in every procedure. The positions of the operating theatre staff and screen position were standardised. Matlab 2017b (The MathWorks, Nattick, MA, USA) software scripts were customized and incorporated in this study to analyse all photographs. These software scripts were used 
to calculate the angles of the shoulders, elbows, wrists, neck and trunk (Figure 1). This was performed for each predetermined step in every single procedure.

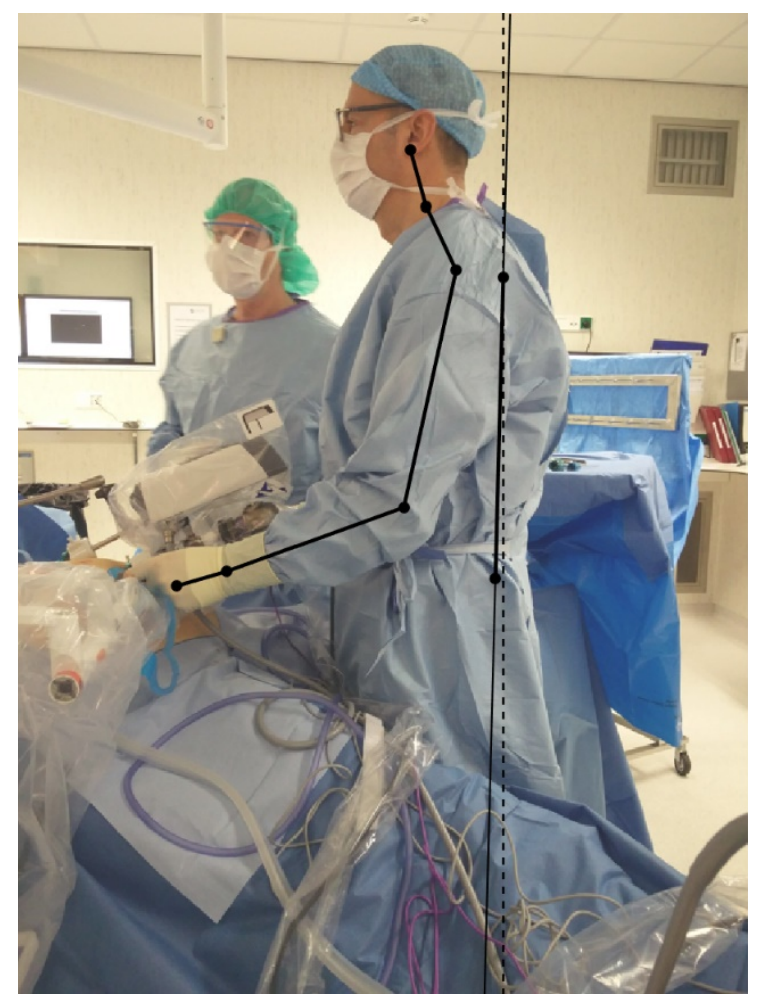

Figure 1: The angles measured using the MATLAB script during a laparoscopic fundoplication.

In addition, all joint angles were converted to the Rapid Upper Limb Assessment score (RULA, Appendix 1). As laparoscopic surgery will generally lead to unilateral strain, the arm with the highest RULA score was incorporated for analysis. The RULA score defines four types of outcome; 1-2 (acceptable posture), 3-4 (further investigation, change may be needed), 5-6 (further investigation, change soon) and 7 (investigate and implement change). Two investigators independently calculated and assessed all RULA scores. Three validated subjective questionnaires were also filled out by all participants in order to assess mental and physical discomfort. (Subjective Mental Effort Questionnaire [SMEQ], National Aeronautics and Space Administration Task Load Index [NASA TLX] and Local Experienced Discomfort scale [LED-scale])

\section{Results}

A total of 30 cases were included. 15 in the AutoLap ${ }^{\text {TM }}$ group and 15 in the control group. All surgical procedures were performed by three surgeons, all enjoying extensive laparoscopic experience and well-trained in employing the AutoLap ${ }^{\mathrm{TM}}$ system. 17 
procedures were performed at the Meander Medical and 13 procedures were performed at the Wilhelmina Hospital. No differences in age or BMI were observed. Mean age was $60.1( \pm 13.4)$ and mean BMI $26.6( \pm 4.1)$. The demographic data of the patients are have been included in Table 1. Five types of surgery were included, i.e. sixteen fundoplications (seven AutoLap ${ }^{\mathrm{TM}}$ cases), seven right hemicolectomies (four AutoLap ${ }^{\mathrm{TM}}$ cases), five sigmoid resections (two AutoLap ${ }^{\mathrm{TM}}$ cases), one rectopexy and one low anterior resection (both AutoLap ${ }^{\mathrm{TM}}$ cases).

Table 1: demographic data of the patients

\begin{tabular}{lllll}
\hline & AutoLap & Control & Total & p-value \\
\hline Age & 60.7 & 59.6 & 60.1 & 0.83 \\
BMI & 27.5 & 25.6 & 26.6 & 0.21 \\
Procedures & & & & \\
Fundoplication & 7 & 9 & $16(53 \%)$ & \\
Right hemicolectomy & 4 & 3 & $5(23 \%)$ & \\
Sigmoid resection & 2 & 3 & $1(3 \%)$ & \\
Low anterior resection & 1 & 0 & $1(3 \%)$ & \\
Rectopexy & 1 & 0 & & \\
\hline
\end{tabular}

The mean RULA score of the surgeon was equal in both groups, $2.58( \pm 0.47)$ in the AutoLap $^{\text {TM }}$ group and $2.72( \pm 0.53)$ in the control group, $p=0.45$. However, the mean RULA score of the first assistant was significantly lower in the AutoLap ${ }^{\mathrm{TM}}$ group, $2.55( \pm 0.54)$ versus $3.69( \pm 0.57), p<0.001$ (shown in Table 2). The intraclass correlation coefficient was 0.93 for the surgeon RULA score and 0.97 for the assistant RULA score.

Subjective questionnaires, including the LED, SMEQ and NASA TLX displayed identical scores for the surgeon in both groups. The mean LED score (post-surgery minus presurgery) for the surgeon was $1.1( \pm 2.30)$ versus $1.6( \pm 2.0)$, mean SMEQ score was 49.7 $( \pm 20.3)$ vs. $43.2( \pm 19.1)$, AutoLap ${ }^{\mathrm{TM}}$ versus control, respectively. The raw NASA TLX was equivalent on all domains (mental, physical, temporal, performance, effort and frustration) in both groups. 
Table 2: The mean RULA scores for the surgeon and assistant

\begin{tabular}{cccccc}
\hline RULA - scores & & Mean & p-value & Standard deviation & $\mathrm{N}$ \\
\hline \multirow{2}{*}{ Surgeon } & Robotic & 2.58 & & 0.47 & 15 \\
& Control & 2.72 & 0.45 & 0.53 & 15 \\
\multirow{2}{*}{ Assistant } & Robotic & 2.55 & & 0.54 & 15 \\
& Control & 3.69 & 0.001 & 0.57 & 15 \\
\hline
\end{tabular}

Table 3: The pre-operative, post-operative and total LED scores and SMEQ scores for surgeons and assistant.

\begin{tabular}{|c|c|c|c|c|c|c|c|c|c|c|}
\hline \multirow[t]{3}{*}{ Questionnaires } & & \multicolumn{2}{|c|}{ SMEQ } & \multicolumn{6}{|c|}{ LED } & \multirow[b]{3}{*}{ N } \\
\hline & & \multirow[b]{2}{*}{ M } & \multirow[b]{2}{*}{ Std } & \multicolumn{2}{|c|}{ Pre } & \multicolumn{2}{|c|}{ Post } & \multicolumn{2}{|c|}{ Total } & \\
\hline & & & & M & Std & M & Std & M & Std & \\
\hline & Robotic & 49.67 & 20.31 & 0.57 & 0.90 & 1.70 & 2.80 & 1.13 & 2.29 & 15 \\
\hline \multirow[t]{2}{*}{ Surgeon } & Control & 43.17 & 19.07 & 0.90 & 1.58 & 2.53 & 2.59 & 1.63 & 2.00 & 15 \\
\hline & $p$-value & 0.37 & & & & & & 0.53 & & \\
\hline \multirow[t]{3}{*}{ Assistant } & Robotic & 28.83 & 23.32 & 4.07 & 9.06 & 4.80 & 11.83 & 0.73 & 3.65 & 15 \\
\hline & Control & 53.33 & 23.45 & 2.20 & 4.04 & 12.67 & 12.97 & 10.47 & 13.18 & 15 \\
\hline & $p$-value & 0.008 & & & & & & 0.010 & & \\
\hline
\end{tabular}

Pre=pre-operative, post=post-operative, total = post-pre, $\mathrm{M=Mean}, \mathrm{Std}=$ standard deviation .

Table 4: The raw NASA TLX with the six domains displayed

\begin{tabular}{|c|c|c|c|c|c|c|c|c|c|c|c|c|c|c|}
\hline & & \multicolumn{13}{|c|}{ NASA-TLX } \\
\hline & & \multicolumn{2}{|c|}{ mental } & \multicolumn{2}{|c|}{ physical } & \multicolumn{2}{|c|}{ temporal } & \multicolumn{2}{|c|}{ performance } & \multicolumn{2}{|c|}{ effort } & \multicolumn{2}{|c|}{ frustration } & \multirow{2}{*}{$\mathrm{N}$} \\
\hline & & M & Std & M & Std & M & Std & M & Std & M & Std & M & Std & \\
\hline \multirow{3}{*}{ Surgeon } & Robotic & 7.70 & 4.09 & 6.23 & 3.41 & 4.63 & 2.57 & 3.30 & 1.58 & 7.90 & 4.74 & 4.57 & 3.71 & 15 \\
\hline & Control & 6.57 & 3.23 & 5.83 & 2.37 & 3.93 & 1.22 & 3.33 & 0.90 & 7.07 & 3.12 & 4.63 & 3.10 & 15 \\
\hline & $p$-value & \multicolumn{2}{|c|}{0.407} & \multicolumn{2}{|c|}{0.712} & \multicolumn{2}{|c|}{0.348} & \multicolumn{2}{|c|}{0.944} & \multicolumn{2}{|c|}{0.574} & \multicolumn{2}{|c|}{0.958} & \\
\hline \multirow{3}{*}{ Assistant } & Robotic & 4.97 & 2.86 & 4.10 & 3.46 & 2.83 & 1.78 & 4.07 & 2.24 & 4.93 & 3.67 & 2.77 & 1.79 & 15 \\
\hline & Control & 7.17 & 3.88 & 9.03 & 4.52 & 5.47 & 2.62 & 6.53 & 3.08 & 8.13 & 3.70 & 4.53 & 2.79 & 15 \\
\hline & $p$-value & \multicolumn{2}{|c|}{0.088} & \multicolumn{2}{|c|}{0.002} & \multicolumn{2}{|c|}{0.003} & \multicolumn{2}{|c|}{0.018} & \multicolumn{2}{|c|}{0.024} & \multicolumn{2}{|c|}{0.048} & \\
\hline
\end{tabular}


For the first assistant, the subjective workload was significantly lower in the AutoLap ${ }^{\mathrm{TM}}$ group for all three questionnaires. The mean LED score (post- minus pre-operative) was $0.73( \pm 3.65)$ versus $10.5( \pm 13.2), p=0.010$, the mean SMEQ score $28.9( \pm 23.3)$ versus 53.3 $( \pm 23.5), p=0.008$, in favour of the AutoLap ${ }^{\mathrm{TM}}$ group (see Table 3 ). The NASA TLX score was significant lower in the Autolap ${ }^{\mathrm{TM}}$ group on all domains except the mental domain. The mean mental score was $5.0( \pm 2.9)$ vs. $7.2( \pm 3.9), p=0.088$, physical $4.1( \pm 3.5)$ vs. $9.0( \pm 4.5)$, $p=0.002$, temporal $2.8( \pm 1.8)$ vs. $5.5( \pm 2.6), p=0.003$, performance $4.1( \pm 2.2)$ vs. $6.5( \pm 3.1)$, $p=0.018$, effort $4.9( \pm 3.7)$ vs. $8.1( \pm 3.7), p=0.024$ and frustration $2.8( \pm 1.8)$ vs. $4.5( \pm 2.8)$, $\mathrm{p}=0.048$. The NASA TLX scores are displayed in Table 4.

\section{Discussion}

The results of this study demonstrate that the ergonomic situation for the first assistant dramatically improves when using a mechanical camera holder, in this case the Autolap ${ }^{\mathrm{TM}}$ system. The disproportional effort of an assistant holding the laparoscopic camera in an unfavourable position is no longer required once a robotic camera holder is installed. Standing in a twisted or stooped position for a longer period, while keeping one hand perfectly still, can be avoided this way. The role of the first assistant changes: now he or she is able to concentrate on tissue traction or presentation of the surgical target area without having to worry about steering and focusing the camera. The ergonomic situation for the surgeon mostly remains unaltered when using the Autolap ${ }^{\mathrm{TM}}$ system. Image quality, however, a very important factor in laparoscopic surgery, may significantly improve. Using a camera holder allows the captured image to continuously remain stable and in full focus, offering a high and consistent image quality. Furthermore, this sees the surgeon now exclusively responsible for steering the camera, with him no longer being dependent on the skills and qualifications of his first assistant. A study conducted by Kavoussi et al. showed less inadvertent camera movements and rotations in the robotic group when the AESOP (Computer Motion system) was employed. [7] A study performed by Proske et al. also reported favourable image quality and stability when using a robotic camera holder.[8] In a large study of 1033 procedures, Holländer et al. reported that eight out of nine surgeons preferred robotic assistance to human assistance, mainly for reasons of the steady imagery this created and surgeons remaining in control of the camera themselves. [9]

Some drawbacks to using a robotic camera holder were, of course, also reported. The system's large dimensions can cause interference with laparoscopic instruments. This can quite often be reduced by spacing the trocar ports at least 8-10 centimetres apart. Future editions of the camera holder should, however, take the size of the camera holding device into account. Furthermore, using a camera holder system limits the camera's reach. The range of motion is often limited to a maximum of 120 degrees, horizontally, and 110 degrees, vertically. This range is not a limiting factor in most of the tasks of a laparoscopic procedure. However, when, for instance, mobilising the splenic flexure during a 
laparoscopic left colectomy, the camera holder will require readjusting during the procedure. This might cause interference to the procedure, which can lead to distress, hindrance or even a delay. Surgeons who are well trained and experienced in using the system will, however, be able to easily overcome these issues.

This study saw RULA scores calculated in an attempt to assess the ergonomic situation of the surgical team members. Several variables had to be taken into account when calculating RULA scores. Certain designated key steps of every surgical procedure were assigned and photographed. Operating theatre staff were not influenced into taking up their natural positions in any way. RULA scores were calculated afterwards. All photos were shot by a single researcher to ensure a standardized method of capturing posture during each procedure. Team member postures could not always be easily to identified in every single photograph. Also of extreme importance in this respect is the angle at which the photo is taken. Once the photo is slightly out from the sagittal or dorsal plain, angle calculations can be difficult to make. That is why second posture analyses would be conducted by an independent researcher. The intra-observer variability was close to 1.0, meaning perfectly corresponding RULA scores were calculated by both researchers.

Another important aspect that needs addressing is a person's natural posture. Natural postures that tend to lean the forward with the head during a procedure, will be scored as ergonomically suboptimal. Only one arm was included on the analysis in this study. There are several reasons for this. In most cases, only one arm was heavily burdened during surgery. In the control cases this always concerned the arm that was holding the endoscope. The LED questionnaire displayed increased scores in body parts on the side of the arm holding the scope. Secondly, in an attempt to not dilute the RULA scores, we excluded the mean of two arms, therefore only including the arm receiving the highest burden into the RULA score.

One also should take into consideration when calculating RULA scores, that maintaining a standing position over a longer period of time will increase the RULA score. That is why higher workload scores tend to be recorded for individuals working in an operating theatre. If accompanied by an ergonomically unfavourable position, scores will then tend to escalate. That is why ongoing attention should continue to be paid to the high physical workload endured in the operating theatre environment. Analysing body postures, formulating suggestions for improvement and gathering evidence that tools such as robotic camera holders can improve operating theatre ergonomics, can yield major future benefits. Paying more attention to a correct working posture during surgery procedures is no longer considered a side issue. It will be one of the key elements to keep the surgical workforce 'fit to perform' over the next decades. [10]

\section{Conclusion}

The use of a robotic camera holder leads to a significant improvement in the ergonomics of the first assistant. Moreover, the subjective work load is reduced by the use of the AutoLap $^{\mathrm{TM}}$ system. The ergonomics and work load of the surgeon remain unaffected. 


\section{References}

1. Nguyen NT, Ho HS, Smith WD, Philipps C, Lewis C, De Vera RM, Berguer R (2001) An ergonomic evaluation of surgeons' axial skeletal and upper extremity movements during laparoscopic and open surgery. Am J Surg 182:720-4.

2. Alleblas CCJ, de Man AM, van den Haak L, Vierhout ME, Jansen FW, Nieboer TE (2017) Prevalence of Musculoskeletal Disorders Among Surgeons Performing Minimally Invasive Surgery. Ann Surg 266:905920. doi: 10.1097/SLA.0000000000002223

3. Alleblas CCJ, Formanoy MAG, Könemann R, Radder CM, Huirne JA, Nieboer TE (2016) Ergonomics in gynecologists' daily practice: A nationwide survey in The Netherlands. Work 55:841-848. doi: 10.3233/WOR-162451

4. Aitchison LP, Cui CK, Arnold A, Nesbitt-Hawes E, Abbott J (2016) The ergonomics of laparoscopic surgery: a quantitative study of the time and motion of laparoscopic surgeons in live surgical environments. Surg Endosc. doi: 10.1007/s00464-016-4855-4

5. Szeto GPY, Ho P, Ting ACW, Poon JTC, Cheng SWK, Tsang RCC (2009) Work-related Musculoskeletal Symptoms in Surgeons. J Occup Rehabil 19:175-184. doi: 10.1007/s10926-009-9176-1

6. Ballantyne GH (2002) The pitfalls of laparoscopic surgery: challenges for robotics and telerobotic surgery. Surg Laparosc Endosc Percutan Tech 12:1-5. doi: 10.1097/00129689-200202000-00001

7. Kavoussi LR, Moore RG, D JBA, And AMS, Partin AW (1995) Urologists At Work COMPARISON OF ROBOTIC VERSUS HUMAN LAPAROSCOPIC CAMERA CONTROL. J Urol 154:2134-2136.

8. Proske JM, Dagher I, Franco D (2004) Comparative study of human and robotic camera control in laparoscopic biliary and colon surgery. J Laparoendosc Adv Surg Tech A 14:345-8. doi: 10.1089/lap.2004.14.345

9. Holländer SW, Klingen HJ, Fritz M, Djalali P, Birk D (2014) Robotic Camera Assistance and Its Benefit in 1033 Traditional Laparoscopic Procedures: Prospective Clinical Trial Using a Joystick-guided Camera Holder. Surg Technol Int 25:19-23.

10. Rosenblatt PL, McKinney J, Adams SR (2013) Ergonomics in the operating room: Protecting the surgeon. J Minim Invasive Gynecol 20:744. doi: 10.1016/j.jmig.2013.07.006 


\section{Appendix 1: RULA score sheet}

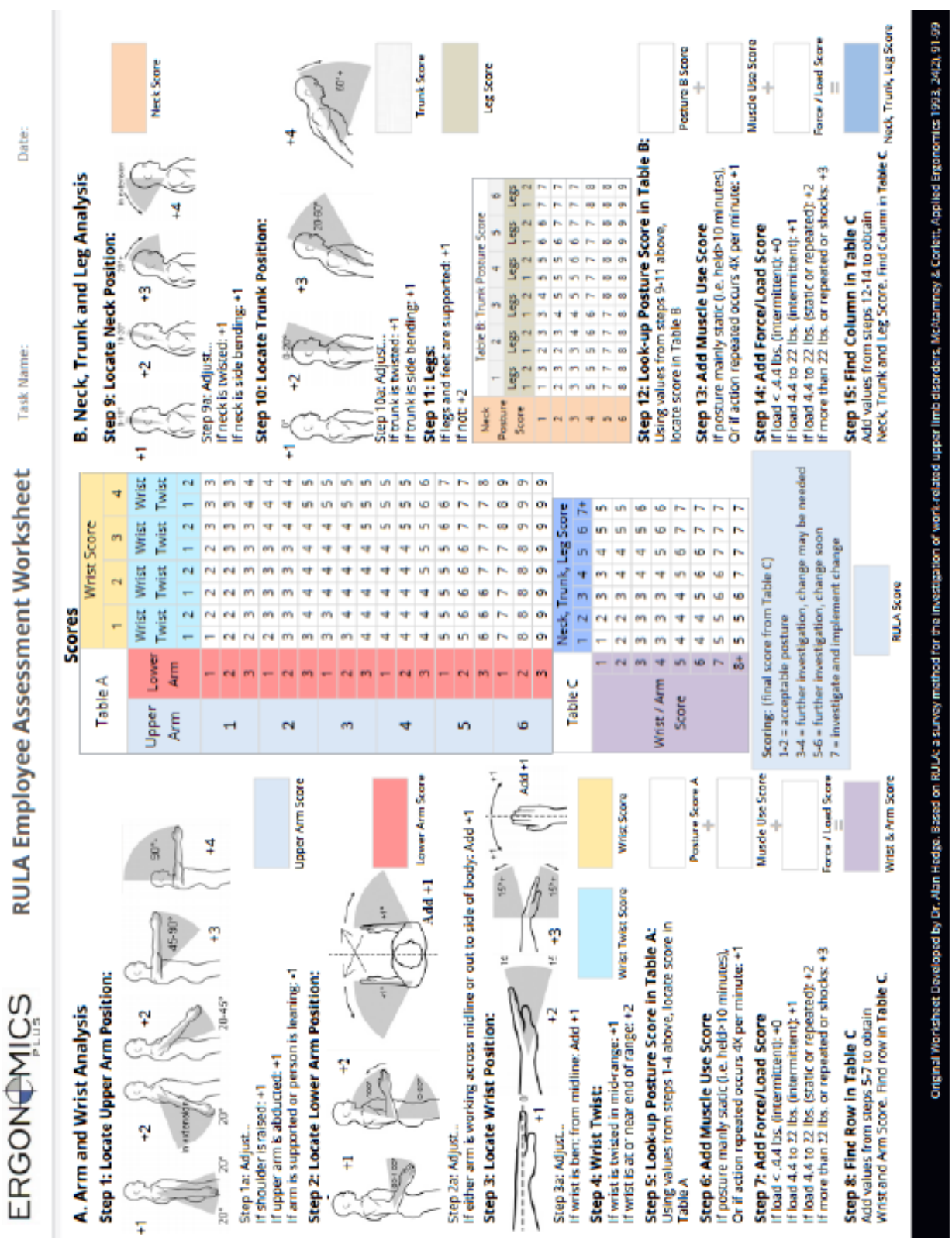


Appendix 2: SMEQ questionnaire

This graphic displays the amount of effort it took you to execute the task you have been working on.

Please score the amount of effort by marking one of the anchors on the vertical line here below.

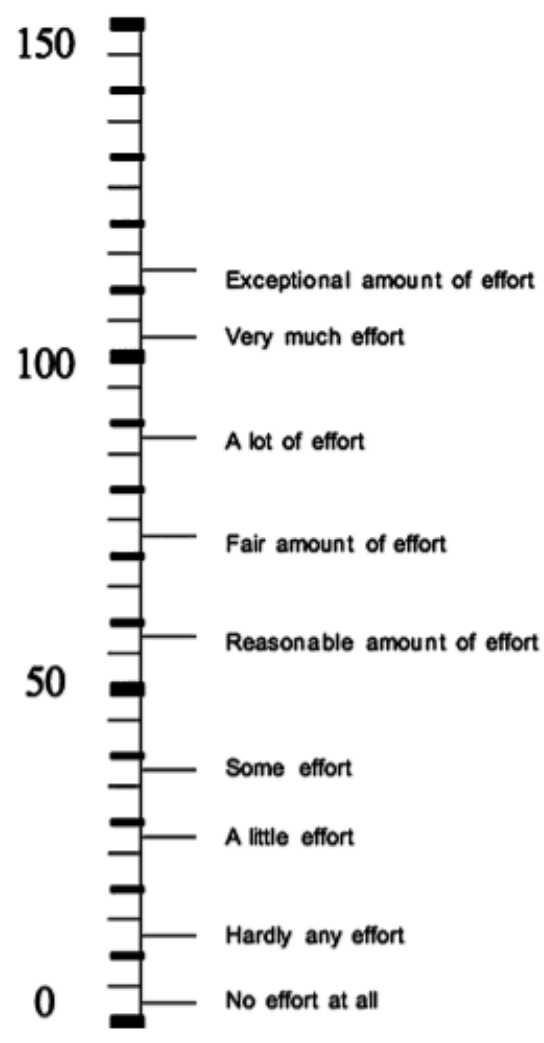




\section{Appendix 3: Local Experienced Discomfort score sheet}

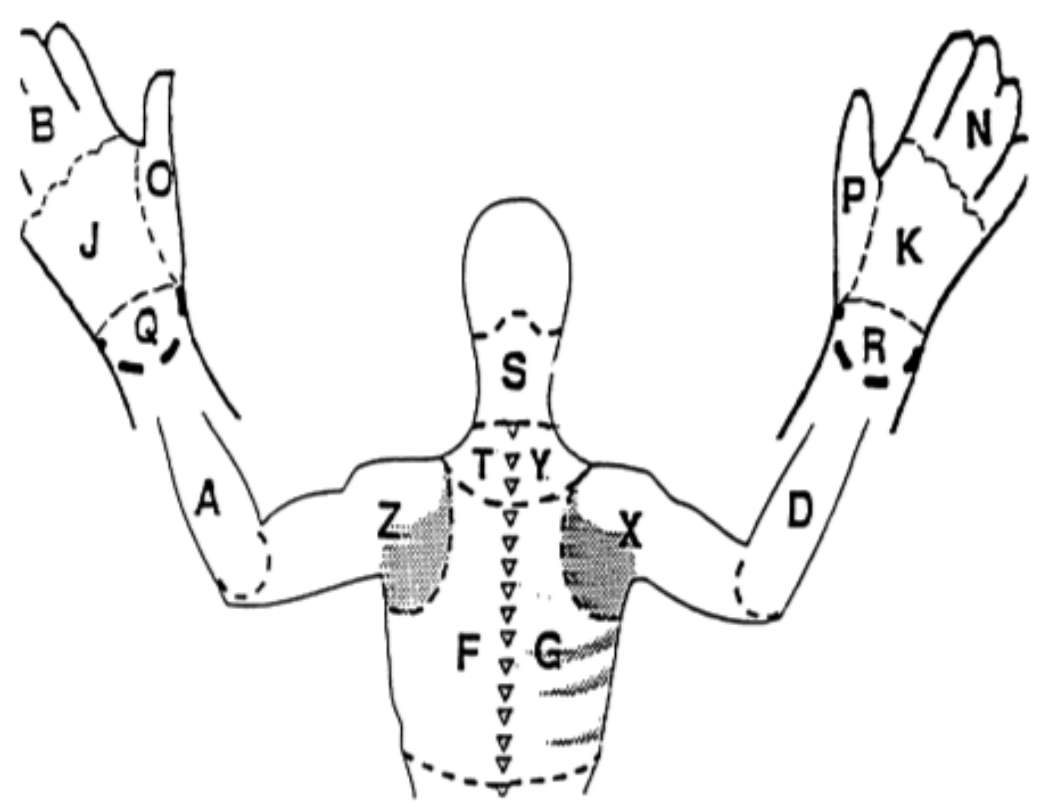

For each of the body parts indicated by the letters in the picture, please fill in a score as presented here below.

Maximum

Extreme amount of complaints

A lot of complaints

Quite a lot complaints

Some complaints

Hardly any complaints

No complaints at all Minimum
Right side Left sido

$\begin{array}{ll}\mathbf{B} \ldots & \mathbf{N} \ldots \\ \mathbf{J} \ldots & \mathbf{K} \ldots \\ \mathbf{O} \ldots & \mathbf{R}_{\ldots .} \\ \mathbf{Q} \ldots & \mathbf{R} \ldots \\ \mathbf{A} \ldots & \mathbf{D} \ldots \\ \mathbf{Z} \ldots & \mathbf{X} \ldots \\ \mathbf{T} \ldots & \mathbf{X} \ldots \\ \mathbf{S} \ldots & \mathbf{S} \ldots \\ \mathbf{F} \ldots & \mathbf{G} \ldots\end{array}$




\section{Appendix 4: NASA Task Load Index score sheet}

Hart and Staveland's NASA Task Load Index (TLX) method assesses work load on five 7point scales. Increments of high, medium and low estimates for each point result in 21 gradations on the scales.

\begin{tabular}{|l|l|l|}
\hline Name & Task & Date \\
\hline
\end{tabular}

Mental Demand How mentally demanding was the task?

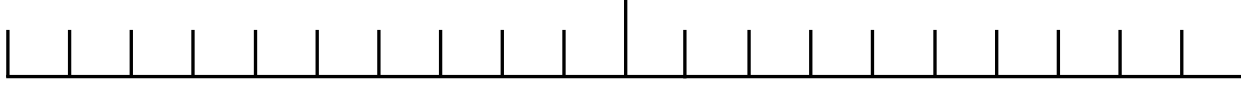

Very Low

Very High

Physical Demand

How physically demanding was the task?

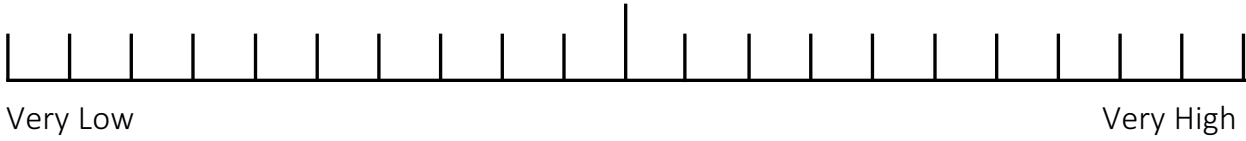

Temporal Demand How hurried or rushed was the pace of the task?

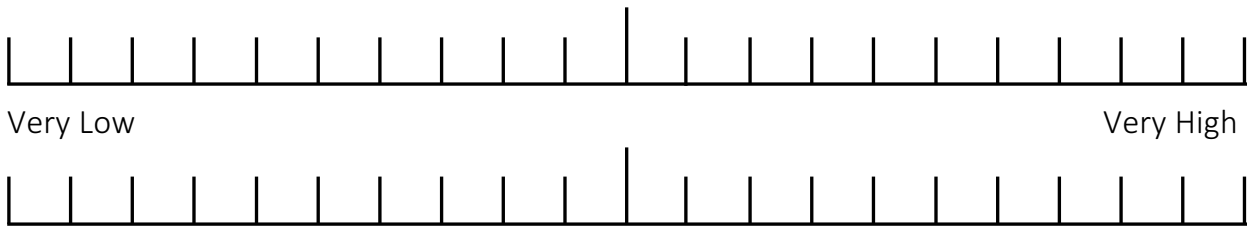

Performance

How successful were you in accomplishing what you were asked to do?

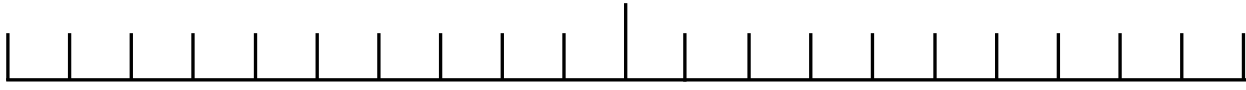

Perfect

Failure

Effort

How hard did you have to work to accomplish your level of performance?

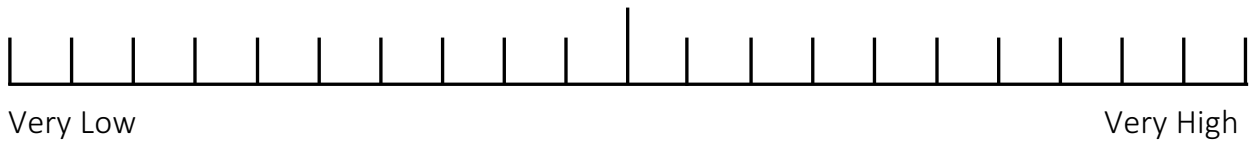

Frustration

How insecure, discouraged, irritated, stressed, and annoyed were you?

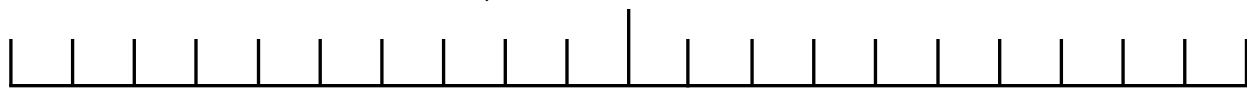

Very Low

Very High 


General discussion and future perspectives

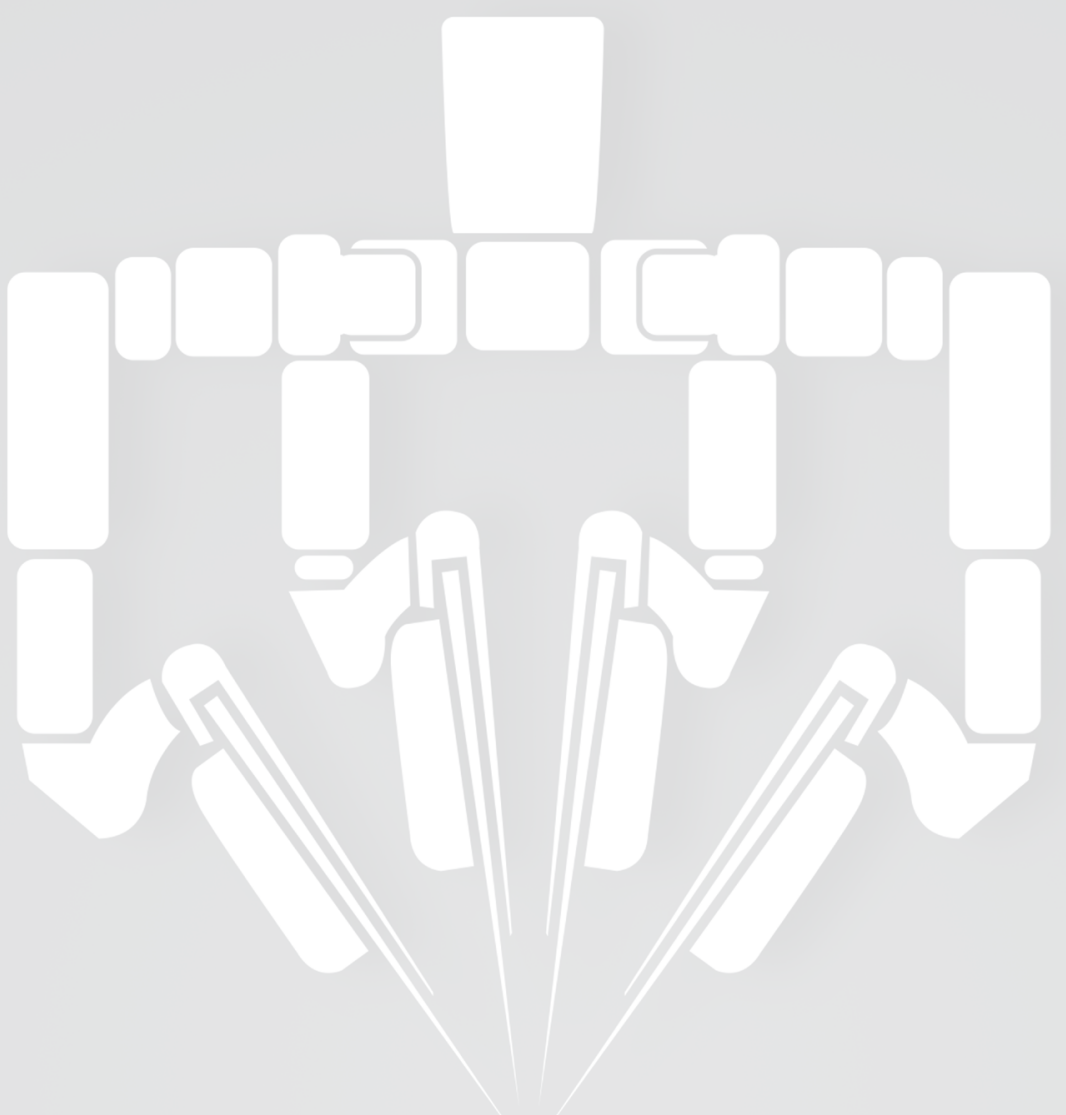



This thesis describes the ergonomic and safety aspects of minimal invasive surgery. The high incidence of musculoskeletal problems that are reported among laparoscopic surgeons emphasises the need for this field of research [1-3]. This thesis is divided into three sections. Part one outlines the introduction of a surgical safety checklist to preparing for minimal invasive procedures. Part two discusses fundamental ergonomics in minimal invasive surgery. Part three discusses advanced ergonomics and the impact of robot-assisted surgery.

The 2000-2015 period saw increased attention given to reducing the number of surgical errors made in operating theatres across the globe. The relevance and importance of checklists and time-out procedures is widely recognized nowadays. A checklist, specifically for minimal invasive surgery, was designed by our research group for actual use in the operating theatre. This thesis discusses the desire for, and the rationalization behind, a surgical checklist. By creating a six-item checklist, many operative hiccups that were encountered during minimal invasive surgery, could be addressed. We have formulated several steps and conditions to contribute to a safer working environment within the operating theatre. When attempting to tackle safety issues need to be tackled, one will often find that sufficient preparation of a task can help overcome many difficulties. In many high-tech industries, such as nuclear power plants and the aviation sector, checklists are much more common than in the field of medicine, or, more specifically, surgery. It was not until the turn of the century that the resistance to using checklists in the medical field began to decline.

Following numerous studies that had found significant benefits to doctors incorporating vital checks into their daily practice, a momentum for change had been created.[4-8] Nowadays, every operating theatre staff member in the Netherlands works with a checklist. Be it for checking an anaesthesia insufflator, or the arrival of a patient at the holding bay, checks are incorporated in daily workflows.

The pitfall in this respect becomes apparent when checklists list excessive detail. The users of checklists boasting excessive detail tend to ignore the less relevant details in such a list. This will reduce its effectiveness. The key is to design a checklist that covers all major safety issues of a surgical procedure, but also avoids all less important details. To avoid a checklist overkill, less important details will need to be left out.

In an environment where checklists are not part of daily routine, the introduction of such a list could meet with severe resistance. The opponents will argue that checklists are time-consuming and lead to an additional administrative workload. In the study described in chapter 3 however, we recorded a reduction in several time slots. The systematic checking of the important items of every procedure, actually made for a quicker start and allowed the workflow to advance more smoothly.

In the near future, digital checks and checklists are likely to either replace or complement manual checks. Biometric identifiers such as iris scanners and fingerprint scanners might be introduced into the operating theatre. These identification systems can be merged with other data, e.g. type of surgery, operating room number, ASA 
classification and other components of the electronic patient file. These novelties have the ability to attain a safer, more efficient and more transparent patient safety monitoring system. Digital systems will replace all paperwork in the operating theatre, including safety checklists like the SURPASS (SURgical PAtient Safety System). Once biometric data are matched with procedure specific characteristics, many of the current checks will vanish. Identification of the patient, checking for allergies and identification of items such as comorbidities can then be generated automatically. However, it will take a lot of changes to infrastructure and information technology to accomplish such a digital transformation. Once such an integrated digital system would work flawlessly, however, many administrative tasks will be eliminated. This will relieve doctors from having to do lots of paperwork and needless checks.

Once all patient, hardware and software items have been addressed, the next phase would be the actual surgical procedure. The high incidence of musculoskeletal problems among laparoscopic surgeons triggered us to address this issue. [1-3] An increased awareness of posture and ergonomics among surgeons is essential to reducing the number of surgeons suffering injury. In attempting to find an ideal starting position, we tried to focus on the ergonomic deficits present at the beginning of every laparoscopic procedure. Using infrared technology, we were able to determine the optimal starting posture for the laparoscopic surgeon. The removal of all regular operating theatre obstacles allowed laparoscopic surgeons to take on their ideal starting position. This has large implications to the surgical procedure. For instance, a change of trocar position during minimal invasive surgery severely affects the ergonomic situation for a surgeon during an entire laparoscopic procedure. By formulating the ideal instrument angle, the surgeon can try to optimize the trocar position in order to work in the optimal configuration. This is likely to lead a decrease in the twisting and turning of the head, the neck, the shoulders and the arms and may therefore reduce musculoskeletal injuries among surgeons.

We expect laparoscopic procedures to increasingly be replaced by robot-assisted procedures. This ushers in a whole different approach to ergonomics in minimal invasive surgery. We conducted a comparative study in order to find out if robot-assisted surgery has the ergonomic and performance benefits it claims it has over laparoscopic surgery (Chapter 5). Several subjective measurements showed severe benefits to using the robotassisted technique. Upon closer inspection of all performance scores, robot-assisted surgery outscored laparoscopic surgery on every single predefined task. The fact that novices have been shown to easily adapt to a new and technically intricate technique, is also promising. Robot-assisted technology is expected to continue its rapid evolvement in the near future. Such technological progress will therefore inevitably lead to improved surgical performance.

There are several potential benefits of robot-assisted surgery, some of which may positively affect ergonomics. A sitting position can reduce fatigue of the legs. Handling control grips is preferred to the use of straight laparoscopic sticks. However, one of the 
major drawbacks of robot-assisted console surgery is the forward leaning position required to control the grips inside the console. Chapter 6 analyses the posture of the sitting position of a surgeon inside the console. Our research team designed specific 2D geometric models with which to calculate the angles in certain joints. This model was able to record potentially harmful neck angles in all participants. The viewer angle of the console can lead to a suboptimal posture. Forward leaning inside the console is essential to achieving adequate stereoscopic vision. This is likely to cause physical complaints for the majority of surgeons working inside the console. A project was undertaken in collaboration with a medical chair manufacturer to address this suboptimal sitting position of the surgeon in the console. After making careful observations in the operating theatre, a special console chair was designed to remedy some of the ergonomic deficits to the current console set-up. We tested several prototypes in an actual operating theatre setting on our way to creating an end model.

Future designs of surgical consoles are likely to negate the need for a forward leaning posture. New systems are likely to be equipped with an integrated (console) chair. Many of the disadvantages to the current set-up are history once the console chair is fitted with a reclining feature. The surgeon's posture is likely to improve once the forward leaning requirement for working inside the console is eliminated. Future robot-assisted systems might therefore boast a totally different set-up. Along with the introduction of integrated augmented reality - probably accompanied by the use of virtual reality headsets - the improvements to the ergonomic situation of the surgeon could be tremendous.

Console surgery in its current set-up is likely to be replaced by new, sophisticated, platforms. Several robotic platforms will be introduced to the market over the course of the next few years. The introduction of the Verb ${ }^{\mathrm{TM}}$ surgical system (Johnson\&Johnson / Google), the Senhance ${ }^{\mathrm{TM}}$ surgical system (Transenterix) and the Hugo surgical system (Medtronic) ushers in a new era of minimal invasive surgery. Attention to the optimal ergonomic position of the surgeon will be crucial in creating favourable selling points for all these systems and in addressing the high rate of musculoskeletal injuries. These systems will for instance be able to incorporate biofeedback systems that can register fatigue in the surgeon. This can potentially lead to prevention of errors caused by a reduced attention span.

Robot-assisted surgery pays little attention to the ergonomic situation of the console surgeon. We have developed a training programme to improve the surgeon's posture inside the console. This programme was put into practice and validated. The effects of a short verbal and written explanation were analysed using a number of means. The master workspace range dropped significantly with the implementation of such a training regime. Also, a lower economy of motion was detected, i.e. a more efficient handling of the console grips. The group that received only standard instructions recorded the same performance scores on all tasks. Moreover, the posture related scores (RULA) dropped significantly in the group that received ergonomic instructions. These results couldn't be reproduced in the needle targeting group. This was most likely the results of other 
aspects of ergonomic performance being studied. RULA scores, however, also dropped significantly in the needle targeting group that had received instructions.

One of the key findings in this research project is that the correct use of the clutch system is essential to reducing the level of musculoskeletal stress to surgeons working in a console. We found that a single coaching session would lead to an immediate drop in RULA scores, without affecting any of the surgical performance scores. This calls for a proper instruction on clutching and ergonomics to be incorporated in every training regime for novice console surgeons. When one knows how to clutch properly, one is likely to the reduce forces on the neck, back and shoulders. The current lack of proper ergonomic instructions and training, should be converted into an extensive training programme for all console surgeons.

A console surgeon will always be accompanied by a scrub nurse, a physician assistant, or a first assistant during surgery. This assistant works in the vicinity of the patient lying on the operation table. When assisting in robot-assisted procedures, the first assistant is tasked with coping with the movements of the robotic arms. Chapter 9 describes a research project set up to address the ergonomics of the first assistant. The actual physical strain of first assistants was identified using questionnaires. The majority of assistants reported severe ergonomic concerns during their work. One out of every five assistants even reported visible bruising, or pain, caused by interference with the robot arms. Tacking was considered as the activity resulting in the highest RULA scores, i.e. this activity incurs the highest objective physical workload. Changing an instrument appeared to also prove a very demanding physical task. Many ergonomic issues can be remedied by a diligent preparation of the procedure. It all starts with an adequate placement of the trocar port. This is very important because the workspace for the first assistant directly correlates with the distance between trocar portals. Furthermore, surgeons also require to have great awareness of the robot arms potentially making abrupt movements when the control grips are moved in a swift fashion. Lastly, an open and clear way of communicating during every case will also reduce the number of harmful situations.

The majority of issues are caused by the movement of the various robotic arms. The need for multiport surgery will be reduced with the introduction of a single port robotassisted system like the da Vinci SP system ${ }^{\mathrm{TM}}$. This can lead to a new situation at the operating table: instrument changes will be less frequent and collision of robot arms will be a thing of the past. Whether a single port robot-assisted system can be made suitable to all minimal invasive surgical procedures, remains an important and interesting question.

A further optimisation of optics will be the next major step in minimal invasive surgery. A crystal-clear, fully stabilised and exactly focussed high definition image should facilitate meticulous surgical dissection. A robotic camera holder is an instrument that can facilitate improved optics. Our research group conducted research into the added value of such a robotic camera holder with regard to ergonomics. This saw two groups compared: one group used a robotic camera holder in several laparoscopic procedures, 
the other used an actual human first assistant to control the camera. RULA scores for the first assistant demonstrated significant lower rates in the AutoLap ${ }^{\mathrm{TM}}$ group, which can be understood as evidencing a better ergonomic situation. Equal RULA scores were found in both groups in respect of the surgeon. As far as all subjective scores derived from several questionnaires are concerned, the robotic camera holder group was found to demonstrate far more favourable results. This means that workload is significantly reduced once the AutoLap ${ }^{\mathrm{TM}}$ system is installed. Drawbacks of the device include its bulky design and the limited angles of view that become available with the use of an active camera holding system. Science is also yet to uncover the optimal steering mechanism for the robotic camera holder. Voice control, eye motion control, joystick control and pedal control are all being looked into by robotic camera holder manufacturers. Smart cameras with augmented reality, able to predict the next area of interest during a surgical procedure, will reduce the need for manual camera steering.

The operating theatre of the future is likely to incorporate more technology as well as augmented reality. Intelligent software will support future surgeons on intricate surgical tasks such as finding a urether in pelvic procedures. Supporting software tools will assist in pointing out anatomical landmarks and critical anatomical structures. For instance, within several years, image overlay techniques combined with augmented reality, are expected to be able to determine tumour margins by the millimetre. Such a system can guide the surgeon during a difficult resection and will lead to less damage to healthy tissue in the surrounding area of the tumour. Also, it will help to avoid irradical resections.

Furthermore, fluorescent techniques for visualising the quality of intestinal or vascular anastomosis are very likely to improve and thus potentially be utilised more often. This is likely to reduce complications and reinterventions. The last decade has seen many technological innovations in minimal invasive surgery. Great progress has been made in that period in the field of surgical stapling alone. More major breakthroughs and new inventions are expected in years to come. Patients will reap the rewards of improvements to technology able to support the surgeon in the best possible manner. According to the law of Moore technological advances do not progress linearly, they progress exponentially; this will lead to a bright future for all fields of medicine, but for minimal invasive surgery in particular.[9] 


\section{References}

1. Filippo Cavallo, Andrea Pietrabissa, Giuseppe Megali, Elena Troia, Stefano Sinigaglia, Paolo Dario, Franco Mosca, and Alfred Cuschieri. Proficiency assessment of gesture analysis in laparoscopy by means of the surgeon's musculo-skeleton model. Annals of surgery, 255(2):394-8, February 2012.

2. K. Miller, M. Benden, a. Pickens, E. Shipp, and Q. Zheng. Ergonomics Principles associated With Laparoscopic Surgeon Injury/ Illness. Human Factors: The Journal of the Human Factors and Ergonomics Society, 54(6):1087-1092, June 2012.

3. Adrian Park, Gyusung Lee, F Jacob Seagull, Nora Meenaghan, and David Dexter. Patients benefit while surgeons suffer: an impending epidemic. Journal of the American College of Surgeons, 210(3):306-13, March 2010.

4. Helmreich RL. On error management: lessons from aviation. BMJ 2000; 320(7237):781-785.

5. Leape LL, Berwick DM, Bates DW. What practices will most improve safety? Evidence-based medicine meets patient safety. JAMA 2002; 288(4):501-507.

6. Helmreich RL, Davies JM. Culture, threat, and error: lessons from aviation. Can J Anesth 2004; 51(suppl_1):R1.

7. Hart EM, Owen H. Errors and omissions in anesthesia: a pilot study using a pilot's checklist. Anesth Analg 2005; 101(1):246-50, table.

8. Verdaasdonk EG, Stassen LP, van der EM, Karsten TM, Dankelman J. Problems with technical equipment during laparoscopic surgery. An observational study. Surg Endosc 2007; 21(2):275-279.

9. Gordon E. Moore, Cramming more components onto integrated circuits. Electronics, Volume 38, Number 8, April 19, 1965 


Summary in Dutch / Samenvatting in het Nederlands 

In dit proefschrift worden verschillende onderzoeken beschreven waarbij de ergonomie van kijkoperaties (minimaal invasieve chirurgie) wordt onderzocht. Dit proefschrift is verdeeld in drie secties. In de eerste sectie wordt de introductie van een checklist, voor chirurgische veiligheid ter voorbereiding op minimaal invasieve procedures, geschetst (hoofdstuk 2-3). Sectie twee beschrijft basale ergonomie bij minimaal invasieve chirurgie. In dit deel worden verschillende onderzoeksprojecten besproken die onderzochten wat de beste houding in een kunstmatige omgeving is. Ook worden de introductie van robot geassisteerde chirurgie beschreven en de effecten daarvan op de houding (hoofdstuk 4-7). In sectie drie worden geavanceerde ergonomie en de impact van robot geassisteerde chirurgie besproken (hoofdstuk 8-10).

\section{Sectie 1}

In hoofdstuk 2 wordt de relevantie en het belang van checklists en time-out procedures geschetst. In de periode van 2000 tot 2015 ontstond de behoefte om het aantal chirurgische fouten in operatiekamers wereldwijd te verminderen. Vele organisaties en inspecteurs waren betrokken bij het creëren van een veiligere en efficiëntere werkomgeving in de operatiekamer. Veiligheidskwesties gaan vaak hand in hand met een voldoende voorbereiding van een bepaalde taak. In dit hoofdstuk beschrijven we de drang en de rationalisatie achter een chirurgische checklist. Ook wordt een voorbeeld getoond voor een dergelijke checklist, specifiek ontworpen voor minimaal invasieve chirurgie.

In hoofdstuk 3 worden de effecten van de implementatie van een preoperatieve checklist onderzocht. Tijdens een onderzoeksproject werden chirurgische fouten en storingen in de operatiekamer gescoord. Het invoeren van een preoperatieve checklist kan enige inspanning en tijd vergen, maar kan zeer nuttig zijn om fouten te voorkomen. Onderbrekingen als gevolg van instrument gerelateerde problemen zijn een kritisch punt van zorg tijdens chirurgische ingrepen. Het kan tijdverlies, irritatie en hinder veroorzaken. Door invoering van de checklist konden deze problemen duidelijk worden verminderd.

Sectie 2

In hoofdstuk 4 beschrijven we een onderzoeksproject dat is uitgevoerd om de optimale houding voor een chirurg die kijkoperaties uitvoert, te achterhalen. In een infrarode laboratoriumomgeving werden alle normale operatiekamer obstakels verwijderd, zoals een operatietafel en apparatuur. Vervolgens werd de positie van de schouders, elleboog en romp van de operateur bepaald. Ook werd een grondige analyse uitgevoerd van de monitor-instrument as, evenals de ideale 
nekpositie. Alle hoeken in de verschillende gewrichten zijn berekend en deze gegevens dienen als basis voor de ideale startpositie tijdens laparoscopische chirurgie (kijkoperaties). De ideale hoek voor de nek is lichte buiging naar beneden. Voor de romp is in de ideale situatie geen rotatie aanwezig. De beste positie voor de armen is licht voorwaarts gebogen en ideale hoek voor de elleboog bedraagt iets meer dan 90 graden buiging. De meest gunstige hoek tussen de instrumenten blijkt, zoals reeds in eerder onderzoek vastgesteld, tussen de 25 en de 30 graden.

Hoofdstuk 5 beschrijft de ergonomische en prestatie verschillen in laparoscopische versus robot geassisteerde chirurgie. Met behulp van een ambulant monitoring systeem werd de fysiologische impact van beide soorten chirurgie op het lijf van de chirurg gemeten. Gedurende verschillende taken, zoals het overpakken van een gemarkeerde draad van links naar rechts en vice versa, werden fysiologische metingen in beide groepen uitgevoerd. Ook werden vragenlijsten afgenomen en werden prestatiescores berekend om een vergelijking tussen de groepen te maken. Deze studie toont aan dat de robot geassisteerde technieken leiden tot een reductie in de fysieke belasting van de operateur. Tevens bleek er, bij alle uitgevoerde taken, sprake van een betere prestatiescore in de groep waarbij robot geassisteerde technologie werd ingezet.

Observaties, onderzoek en voorkeuren van chirurgen hebben geleerd dat het werken in een cockpit ofwel console tijdens robot geassisteerde chirurgie, de ergonomie verbetert in vergelijking met laparoscopische chirurgie. Echter, ook bij het werken in de console, kunnen er klachten bij chirurgen ontstaan. Bij veel operaties werd een suboptimale zitpositie van de chirurg waargenomen. Een van de belangrijkste nadelen is de voorwaartse buig- of leunende positie die nodig is om de twee hendels voor telemanipulatie in de console te kunnen besturen. Daarom gaat de zoektocht naar optimale ergonomie verder in hoofdstuk 6. Dit hoofdstuk beschrijft een grondige analyse van de zithouding van een chirurg in de console. Door een 2D geometrisch model te gebruiken, zijn berekeningen gemaakt om hoeken in specifieke gewrichten van de operateurs te kunnen bepalen. Hierbij werd vaak een suboptimale houding van de chirurg vastgesteld. De reden hiervoor ligt in het ontwerp van de console, gecombineerd met de instelmogelijkheden.

Momenteel is er geen officiële stoel die bij de console van het da Vincirobotsysteem wordt geleverd. Daarom is er een onderzoeksproject opgezet om de specifieke kenmerken te bepalen die essentieel zijn voor het optimaliseren van de zithouding van de chirurg. In hoofdstuk 7 wordt beschreven hoe een speciale consolestoel voor het da Vinci-robotsysteem werd ontworpen. Dit gebeurde in samenwerking met een bedrijf dat medische stoelen produceert. In 
dit project is geprobeerd verschillende ergonomische tekortkomingen in de huidige console-instellingen te compenseren en worden verschillende prototypes beschreven van de stoel.

Sectie 3

In deel drie van dit proefschrift wordt een analyse van de ergonomie van robot geassisteerde chirurgie beschreven. Dit begint met hoofdstuk 8 , waarin een trainingsprogramma werd opgezet en gevalideerd. Aan het project werkten artsassistenten en coassistenten mee. In een trainingsprogramma werd een korte uitleg over ergonomie gecombineerd met trainingsoefeningen en aanvullende uitleg. De deelnemers voerden twee sets van trainingsoefeningen uit op de da Vinci vaardigheden simulator. Alle prestatiescores werden berekend. Ook werd er met behulp van vragenlijsten naar de fysieke klachten van de deelnemers gekeken. Deze groep werd vergeleken met een andere groep die alleen standaardinstructies ontving. De groep die de korte coaching sessie doorliep, toonde betere ergonomische scores. In de controle groep werden frequent suboptimale zitposities vastgesteld, die weer kunnen leiden tot fysieke klachten. In de prestatiescores werden geen verschillen tussen beide groepen aangetoond.

In hoofdstuk 9 wordt een heel ander aspect van het werken met robot geassisteerde chirurgie belicht. Terwijl de instrumenten op de uiteinden van de robotarmen worden bestuurd door de chirurg, is er altijd een eerste assistent of een operatieassistent aanwezig die bij de operatietafel in het steriele werkveld direct naast de patiënt staat. Deze assistent speelt een cruciale rol in elke chirurgische robotingreep. Instrumenten en naalden moeten worden vervangen, staplers en tackers (chirurgische nietapparaten) worden door hen afgevuurd. Een arts of verpleegkundige die bij een dergelijke procedure assisteert, moet kunnen omgaan met de bewegingsuitslagen van de robotarmen. De robotarmen kunnen interfereren en hinder veroorzaken tijdens assistentie van een procedure. Dit onderzoeksproject beschrijft de ergonomie van de eerste assistent bij robot geassisteerde chirurgie. Voor dit project werden vragenlijsten afgenomen en werden objectieve operatiekamermetingen gescoord door een waarnemer. Concluderend kan gesteld worden dat er veel valt aan te merken op de ergonomische belasting voor de eerste assistent. Er worden vaak extreme hoeken in gewrichten gemeten, met name tijdens taken zoals tacken, tractie geven op weefsel en het verwisselen van instrumenten. Het hoofdstuk wordt afgesloten met verschillende adviespunten voor het chirurgisch operatieteam.

Het laatste hoofdstuk, Hoofdstuk 10, beschrijft de rol van een robotische camerahouder bij laparoscopische chirurgie. Het doel van dit project was om de impact uit te zoeken van een dergelijk apparaat op de dynamiek en ergonomie 
van een minimaal invasieve chirurgische ingreep. Door een gerandomiseerde studie uit te voeren, werden twee groepen gemaakt. Eén groep voerde alle procedures uit met behulp van een assistent die de camera bestuurde. In de andere groep werd steeds gebruik gemaakt van de robotische camerahouder. Beide groepen werden gescoord door het meten van hun RULA-scores, die werden afgeleid van foto's op de operatiekamer. Ook hebben chirurgen en hun assistenten vragenlijsten ingevuld. Met deze gegevens kon objectief worden vastgesteld dat het gebruik van een robotische camerahouder een zeer gunstig effect heeft op de ergonomie van de eerste assistent. Tevens bleek uit de vragenlijsten dat de gebruikerservaring van zowel chirurg als assistent positief was. De workload voor de chirurg bleef in beide groepen gelijk. 




\section{List of publications}

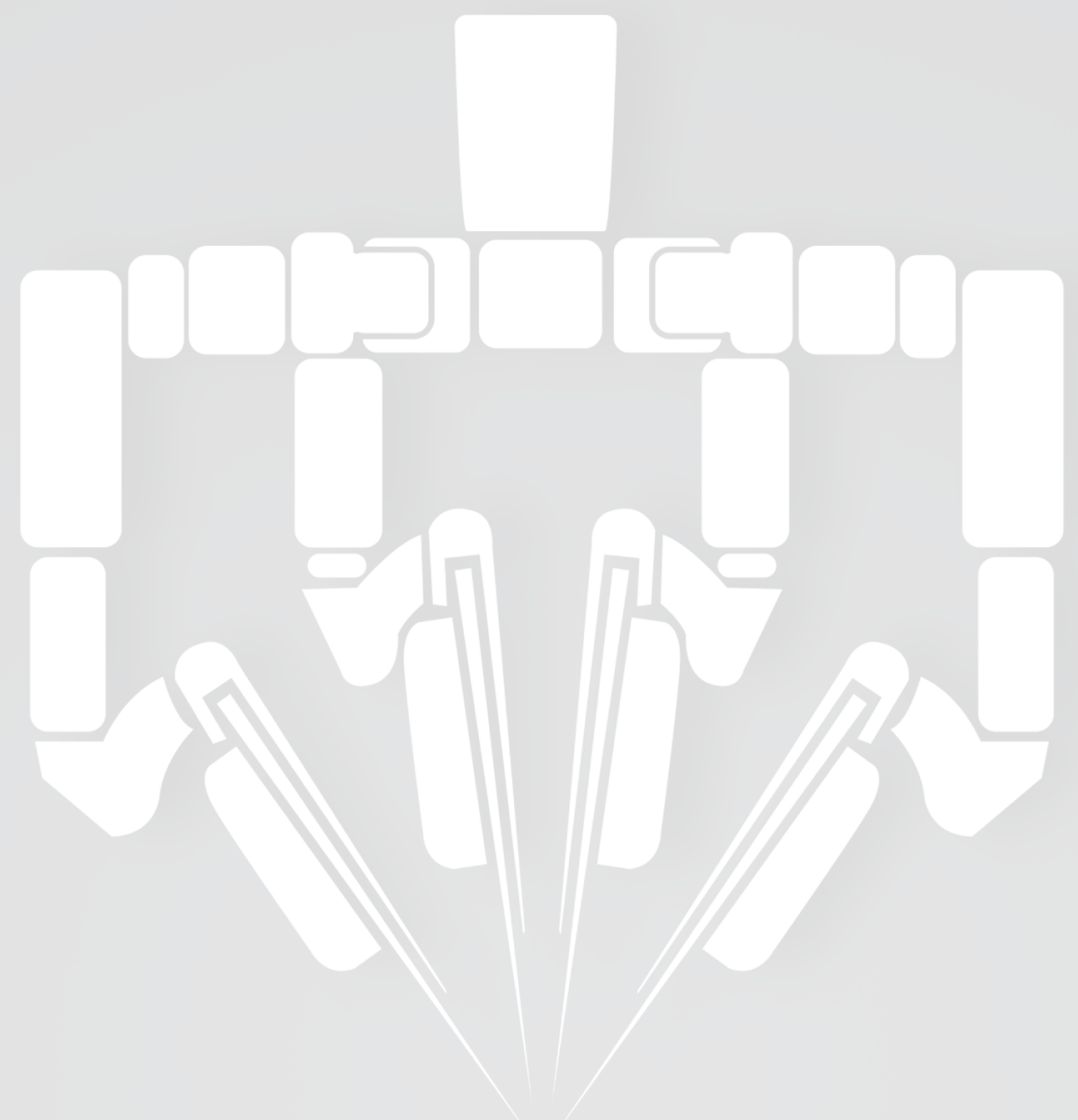



'Renal function after endovascular aortic aneurysm repair: a single-center experience with transrenal versus infrarenal fixation.'

Waasdorp E, Van 't Hullenaar CDP, Van Herwaarden J, Kelder H, Vd Pavoordt E, Overtoom T, Moll F, De Vries JP.

Journal of Endovascular Therapy, 2007, April;14(2):p130-7.

'Checklists \& time-out procedures on the endoscopic operation room'

Van 't Hullenaar CDP, Consten E, Broeders IAMJ

Textbook of Endoscopic Surgery (Dutch edition), April 2009, chapter 24: p169-72

'Ergonomics, user comfort and performance in standard and robot-assisted laparoscopic surgery.'

Van der Schatte Olivier R, Van 't Hullenaar CDP, Ruurda JP, Broeders IAMJ.

Surgical Endoscopy (2009) June; 23(6):p1365-71

'Abdominal pain after penetrating trauma of the upper leg'

Van 't Hullenaar CDP, Voorhoeve R

Annals of the Dutch Surgical Society (NVvH), December 2010, 19 (9): p348-51

'Ileostomy versus colostomy: which is preferable?'

Amelung FJ, Van 't Hullenaar CDP, Verheijen PM, Consten ECJ

Dutch Journal of Medicine (NTVG) 2017;161(0):D788.

'Ergonomic assessment of the da Vinci console in robot-assisted surgery'

Van 't Hullenaar CDP, Hermans B, Broeders IAMJ

Innovative Surgical Sciences (ISS, 2017), Volume 2, Issue 2, p97-104

'Validation of ergonomic instructions as part of Da Vinci simulator training' Van 't Hullenaar CDP, Mertens AC, Ruurda JP, Broeders IAMJ

Surgical Endoscopy (2018) May; 32(5):p2533-2540.

'Ergonomic assessment of the first assistant during robot-assisted surgery' Van 't Hullenaar CDP, Bos J, Broeders IAMJ

Journal of Robotic Surgery (2018) Jul; 24.

DOI: 10.1007/s11701-018-0851-0 [Epub ahead of print] 

Curriculum vitae auctoris

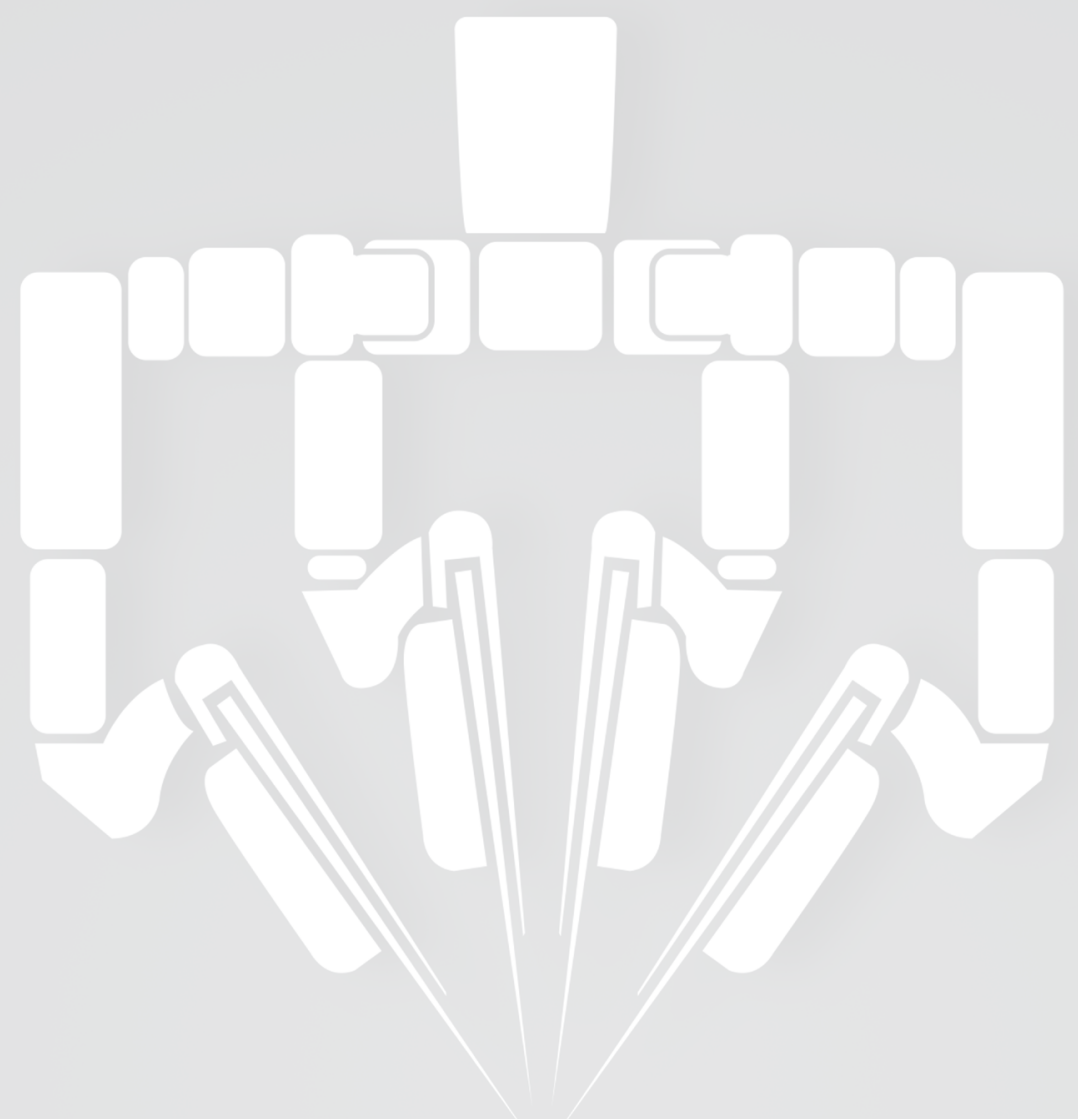



The author of this thesis, Casper Dirk Paul van 't Hullenaar, was born in Eindhoven, the Netherlands on August 8, 1980. He graduated from secondary school (VWO, Athenaeum) at the Van Maerlant Lyceum in Eindhoven, the Netherlands, in 1999. That same year, he enrolled at The Thim van der Laan Academy in Utrecht, the Netherlands. He completed this fast-track training in Physical Therapy in the summer of 2002.

In September 2002 Cas was allowed to enrol at medical school at Utrecht University. In 2006 he served an apprenticeship as a medical intern at the Department of Paediatrics at L'Ospedale San Paolo, Milan, Italy. In his final year at medical school, he spent three months working on a research project in the operating theatre at Weill Cornell Medical Centre, New York, NY, USA. This was his first solo research project and looked at operating room safety checklists. He conducted his research under the supervision of Professor Broeders, Professor Borel Rinkes, Professor Pomp and Associate Professor Dakin. The project won the Concilium Chirurgicum Neerlandicum prize in 2007.

Upon finishing medical school in February 2009, Cas joined the Department of Surgery, Meander Medical Center at Amersfoort, for a year as a junior surgical resident under the supervision of Dr. Van Overbeeke. Having briefly worked at the Medical Skills Lab at Utrecht University, Cas started working as a junior resident under the supervision of Dr. Pluim at the Intensive Care Unit of the Meander Medical Center in September 2010.

His basic surgical training commenced in 2011 at the University Medical Center in Utrecht under the supervision of Professor Borel Rinkes and Professor Vriens. In 2013, Cas started working as a senior surgical resident at the Meander Medical Center in Amersfoort under the supervision of Dr. Consten and Dr. Verheijen.

In April and May 2018, Cas travelled to Brisbane and Sydney, Australia on a WCP / ESCP Travelling Fellowship Grant to visit eight Australian expert centres in colorectal surgery. Upon completing his surgical training in the summer of 2018, Cas will move to take up the post of senior clinical fellow of colorectal surgery at the Oxford University Hospital in the United Kingdom.

During his time as surgical resident, he initiated several research projects in collaboration with the Faculty of Technical Medicine of Twente University, all of which were supervised by Professor Broeders. These projects have culminated in this thesis on ergonomics and safety in minimal invasive surgery.

Cas lives in Utrecht, the Netherlands and is married to Vivian. They have two daughters, Lenthe and Sterre. Independent of the weather conditions, Cas likes going out for a ride on his racing bike several times a week. During winter he likes to visit the Alps for snowboarding and in summer he likes to visit the Frisian lake district or the seaside with his family for some relaxation and water sports. 



\section{Dankwoord / Acknowledgements}

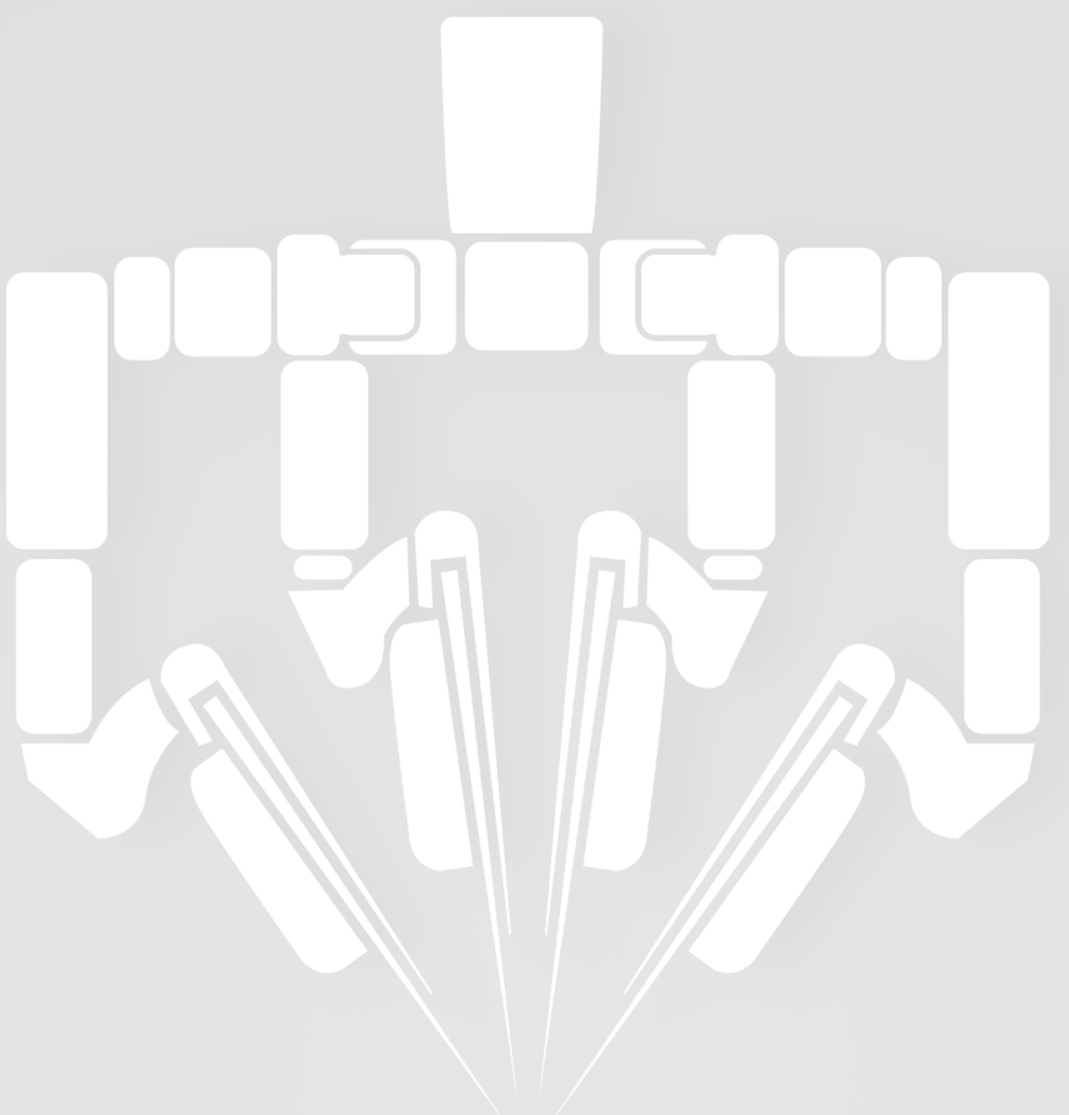



Prof. dr. Broeders, beste Ivo

In 2007 kwam ik als snotneus aankloppen bij jouw kantoor in de stafgang van het AZU. Enkele dagen daarvoor had ik vol bewondering geluisterd naar een loeistrakke presentatie over de operatiekamer van de toekomst. Na een kort gesprek was mijn participatie in het eerste onderzoeksproject een feit. Je had een mooie testcase voor me. Er was een onderzoeksproject dat nieuw leven moest worden ingeblazen. Gelukkig had ik net mijn basic life support behaald en samen met Roelof van der Schatte Olivier en Jelle Ruurda werd er binnen een paar maanden een mooi artikel geschreven. We hadden meteen een goede publicatie te pakken. Deze vliegende start was een mooi begin van een langdurige samenwerking, zowel in de wetenschap als wel in de kliniek. In de jaren die volgden, heb ik deze vliegende start niet bij elk researchproject structureel toegepast. Sterker nog, tijdens verschillende projecten is jouw geduld regelmatig op de proef gesteld. Verschillende reanimaties waren nodig om onze artikelen er doorheen te slepen. Maar door jouw grenzeloze vertrouwen, gedurfde vastberadenheid en passie voor dit onderwerp, wisten we steeds weer een nieuwe invalshoek te vinden voor dit mooie promotietraject. Je vlammende en inspirerende speech in Kirchberg was een betoog dat hierbij mooi aansluit. Ik dank je zeer voor al je inspanningen als mentor, pater familias, coach, inspirator en gids. Als chirurg, professor en promotor ben je een voorbeeld voor velen en in het bijzonder voor mij. Verrekte veel dank!

Dr. Ruurda, beste Jelle, Jouw passie voor de minimaal invasieve chirurgie weet je te combineren met een veelheid aan researchprojecten. Zowel op de operatiekamer, in de research als op het sportveld sta jij je mannetje. Jouw input was cruciaal bij meerdere artikelen in dit proefschrift. Met snelle en glasheldere commentaren hielp je mij een paar keer op het juiste pad. Hoe anders was dit in Ischgl, toen we onder jouw bezielende leiding terechtkwamen in een couloir zonder uitgang. Ik kwam met mijn atletische lijf niet meer vooruit in de metersdiepe Tiefschnee en was daarom genoodzaakt om in buikligging op mijn snowboard mijn weg te vervolgen. Een legendarische middag! Je bent een inspirerende chirurg en een begenadigd onderzoeksbegeleider. Ik vind het een eer dat jij vandaag mij wil bijstaan als copromotor en ik hoop in de toekomst nog eens een artikel met je te schrijven, te opereren of van een piste af te knallen! Many Thanks!

Geachte leden van de beoordelingscommissie: Prof. dr. Borel Rinkes, Dr. Van Det, Prof. dr. ir. Hermens, Dr. Horeman, Prof. dr. ir. Koopman, Prof. dr. Meijerink,

Zeer hartelijk dank voor uw interesse, tijd en energie die $u$ in de beoordeling van dit proefschrift heeft willen steken. Tevens ben ik $u$ allen zeer erkentelijk voor het plaatsnemen in de oppositie. 
Dr. Consten, lieve Esther,

Waar moet ik beginnen? Vanaf het moment dat ik een onderzoeksproject mocht komen doen in Ziekenhuis Lichtenberg te Amersfoort, hadden we een klik. Jij had vervolgens altijd nog wel een klusje voor me liggen of een researchproject voor me open staan. "Luister, vriend! Als jij die professor in New York nou eens even een mailtje stuurt?"...en zo gezegd, zo gedaan. Zonder jouw inspanningen was ik nooit gekomen op de plek waar ik nu ben. Je hebt mij altijd onvoorwaardelijk gesteund en me door soms lastige tijden geloodst. En nu kan ik bijna mijn C-papiertje in ontvangst nemen: echt geweldig! Dit proefschrift is daarbij natuurlijk helemaal een prachtige kers op de taart! Mede met jou als drijvende kracht ben ik de halve wereld over gereisd, heb ik inspirerende mensen en chirurgen ontmoet, talloze congressen bezocht en de mooiste verhalen verzameld. Bovendien heb jij als opleider in het Meander Medisch Centrum een klimaat gecreëerd waarin talent kan uitblinken en de sfeer echt uniek is. Ik bewonder je uitzonderlijke drive en je aanstekelijke enthousiasme! Ik zal mijn best doen om steeds "alle ballen in de lucht te houden", want dat is toch de kern van ons mooie vak! Trek nou maar snel die hele enchilada open!

Dr. Verheijen, beste Paul,

Zal ik de 'Eye of the Tiger' nog één keer opzetten als jij de OK oploopt? Of wordt het toch Lee Brice met 'I drive your truck'? Paul: de man, de mythe. De afgelopen jaren heb ik echt ongelofelijk veel van je geleerd! Niet alleen over uitstekende countrymuziek, maar ook over fistels, littekenbreuken, colorectale chirurgie, betweterige managers, complexe patiënten met nog complexere enterocèles, BMW sportvelgen, wielrennen, Harmonic scalpels, moeilijke en makkelijke vlakken, koffie in Baarn en vele andere zaken die het leven zo schitterend maken. Jouw wijsheid en skills geven mij veel reden tot bescheidenheid. Ik dank je zeer voor het overbrengen van al jouw kennis en voor alle leerzame en beregezellige operaties en poli's!

Prof. Borel Rinkes, beste Inne,

Dank voor het vertrouwen en dank voor alle support! Mede door jouw steun kon ik starten met de opleiding Chirurgie, het allermooiste vak dat er is. Je stimuleerde me om onderzoek te blijven doen en bent immer geïnteresseerd in alle AIOS van Regio $\mathrm{V}$, echt grandioos! Tot op het Heelkundediner, een congres op Ibiza of een spinfiets op de Biltstraat!

Dr. Draaisma, beste Werner,

Altijd op het scherpst van de snede! Jouw passie voor de (minimaal invasieve) chirurgie heeft je al ongelooflijk ver gebracht, zowel op klinisch als wetenschappelijk gebied. Zeer bewonderenswaardig! Ik heb veel respect voor de wijze waarop jij altijd perfectie probeert na te jagen en nieuwe technieken razendsnel adapteert. Ik heb ontzettend veel van je geleerd. Dank hiervoor! 
Dr. Dakin and Professor Pomp, dear Greg and Alphons,

A decade ago, I was a young medical student from the Netherlands. After my presentation about checklists in the operation room at the SAGES conference in Philadelphia, we set up a meeting to talk about a larger research project. You invited me to set up an interesting research project in your outstanding clinic. My time at the Weill Cornell Medical Centre significantly contributed to this thesis. We undertook several attempts to have our results shared in medical journals, but often found publishers reluctant to publish operating room data only. Given the importance of this subject, I would say they missed some excellent opportunities. Operation team dynamics and 'flow' during surgical procedures are currently vibrant fields of research, and a thorough checklist is now mandatory on every surgical procedure. I guess we didn't have that end punch and the momentum needed for a publication, but I'm proud to nevertheless see the results of our study now incorporated into this thesis. You supported me both during my time in New York and later on, giving this research project and my surgical career a real 'Big Apple' boost. I thank you both very much. Please send my love to everyone at NYP/Cornell!

Dr. Clark and Professor Stevenson, dear David and Andrew,

Thank you very much for your kindness and overwhelming hospitality during my stay in Brisbane! I had a wonderful time and witnessed some amazing minimal invasive surgeries. I'm sure your clinic will remain a world-class institute as long as you guys are in charge there! I'm sure our roads will cross again. Cheers to that!

Dr. Jones, Dr. Cunningham and Dr. Lindsey, dear Oliver, Chris and lan,

Many thanks for the opportunity of starting out at your clinic this coming autumn! I'm looking forward to beginning my senior colorectal fellowship, as I am hopeful of positively contributing to the colorectal patient care and research at Oxford University Hospital. See you all next month!

Alle stafleden, fellows, arts-assistenten van het UMC Utrecht en het Meander Medisch Centrum,

Dank allen voor jullie steun en vertrouwen in mij! Twee mijlpalen in één week! Is het niet grandioos? De afgelopen jaren heb ik met veel van jullie een band opgebouwd die uniek is op de werkvloer. In ons vak moet je elkaar kunnen vertrouwen. Je moet varen op het oordeel en de vaardigheden van je collega's. Jullie hebben mij altijd vertrouwd en ik kon ook altijd op jullie vertrouwen. Ik heb genoten van onze bijzondere teamspirit, die op de jaarlijkse apotheose in de Alpen altijd weer goed tot zijn recht komt! Mede dankzij jullie grandioze inzet levert de vakgroep Chirurgie elke dag uitstekende zorg op de afdelingen, de poliklinieken, de spoedeisende hulp en de operatiekamers van Utrecht en Amersfoort. Ik dank jullie voor alle schitterende jaren! En natuurlijk special thanks aan mijn roosterbuddy's: Marjolein Blussé van Oud Alblas, Okan Bastian (en wat een prachtige kaft, I love your skills, thanks!), Henk Formijne Jonkers en Anne den Hartog. 
Alle operatie assistenten van het Meander Medisch Centrum,

Met in het bijzonder Merilyn Bruins, Perla Doornbos, Merel Methorst, Rianne Sonneveld, Enrico van Delden, Eveline van Zuijlen, Ronald Dorrestein, Angelique Koster, Kim van Tuyl en alle andere leden van het robotteam die ik nu ongetwijfeld nog vergeet. Jullie bijdrage aan de studie over de ergonomie van de operatieassistent was grandioos! Daarnaast zal ik de inzet, de gastvrijheid, de loyaliteit, de humor en de klasse van het gehele operatiekamerpersoneel (ja, zelfs van de anesthesie!) in het Meander Medisch Centrum nooit vergeten: dank jullie allemaal zeer voor vijf grandioze jaren!

Het Meander Sterrenteam: thanks voor alle hardloopsessies langs de Eem en alle koersetappes door de Soestduinen. Ik zou zeker 130 kilo wegen als ik jullie niet had gehad!

Beste Maarten van Alphen en Matthijs Hendriks,

Zeer bedankt voor jullie inspanningen met betrekking tot het onderzoeksproject waarin we de ideale laparoscopische operatiehouding hebben beschreven. Tientallen meetsessies in het infraroodlaboratorium hebben we doorstaan! Daarna volgden er uitgebreide analyses waarbij jullie mij steeds probeerden uit te leggen hoe de X-, Y- en Zvector correct geplot konden in een 3D-model. Leuk om te zien dat jullie hard aan de weg timmeren en de medische wereld blijven verbinden met een sprankelende technologische toekomst!

Beste Roelof van der Schatte Olivier,

Met jouw project is het allemaal begonnen! Samen bliezen we een artikel nieuw leven in en zo begon mijn reis bij de Chirurgie. Jouw reis in de Geneeskunde heeft je ook al op veel plekken gebracht. Via een uitstapje bij Defensie ben je nu op een mooie opleidingsplek bij de Anesthesiologie. Dank voor het prachtige vonkje dat dit vuurtje heeft ontstoken!

Beste Ben Hermans,

Jij bent de grondlegger van de complexe hoekmetingen op de operatiekamer! De RULA scores kwamen keer op keer soepel uit jouw MATLAB scripts rollen. Je hebt een goed gevoel voor grafische weergave van resultaten en je hebt in korte tijd een topprestatie geleverd! Ergonomie kan altijd beter en dat hebben we mooi laten zien in ons artikel. Ik wens je veel succes met de afronding van je promotietraject aan de Universiteit van Maastricht, waar jij de Cardio-elektrofysiologie ongetwijfeld naar een hoger plan tilt!

Lieve Paula Bos,

Jij verdient ook zeker een speciale vermelding! Met jouw ongekende inzet hebben we een lastig onderwerp goed op de kaart gezet. Je kwam voor een korte stage, maar bouwde supersnel met iedereen op de Robot OK-5 een band op! Wekenlang heb je elke beweging van de robot en de operatieassistenten nauwlettend geobserveerd en gescoord. Dit heeft een prachtig artikel opgeleverd. Jij hebt zelfs tot lang na je 
stageperiode veel betrokkenheid getoond bij dit project en ik heb veel respect voor je doorzettingsvermogen! Veel dank en succes met je promotietraject bij de Hoofd-hals oncoradiologie van het Antoni van Leeuwenhoek Ziekenhuis!

Beste Hans Blankenvoort,

De wijze waarop jij als industrieel ontwerper naar vormgeving, ergonomie en design kijkt, vind ik zeer inspirerend! Je hebt je maandenlang volledig ondergedompeld in de chirurgische wereld en wist je snel te bekwamen in de dagelijkse medische praktijk. Ook kon je snel de link leggen naar praktische oplossingen bij het ontwerp van een robotstoel. Helaas is ons octrooi er nooit van gekomen, maar de bewustwording van zithouding in de console heb jij prachtig vertaald naar een mooie stoel en een schitterende afstudeerscriptie! Heel tof dat dit project heeft geresulteerd in je huidige baan bij SCORE!

Beste Sander Mertens,

Heel veel dank voor jouw fantastische werk aan het hoofdstuk over het verbeteren van de ergonomie van de console. Jij hebt je vol enthousiasme gestort op een complex onderwerp. Met vele uren metingen op Robot OK-5 en eindeloze analyses, heb jij bewezen dat je een onderzoeksproject tot een prachtig eindresultaat kunt brengen! Ik wens je ontzettend veel succes met alle nog lopende studies de komende jaren en ik wens je een mooie carrière voor daarna! (tja, want je ziet wat er gebeurt als je het tegelijk doet...)

Beste Paul Wijsman,

AUTOLÂP!!!! Wie Autolap zegt, zegt Paulus Wijsman. Gozer, wat heb jij de afgelopen jaren een ontwikkeling doorgemaakt. Maar je blijft nog steeds die heerlijke nuchtere man uit het Oosten die gek is met een potje bier en de Zwarte Cross. Dankzij jou weet ik wat ik wat brommers kieken, høken en een keet is: toch wel een serieuze verrijking zou ik zo zeggen. Met jouw pientere blik wordt research een heel stuk leuker! En als jij heerlijk bijdehand en onverschrokken aan een ouderejaars AIOS uitlegt hoe de ductus cysticus het beste kan worden vrijgelegd, kun je mij wegdragen! Je bent een topper, Paul! Ga zo door!

Technisch Geneeskunde-onderzoekers Lennert Molenaar, Gert Jan Snel, Omar Hertgers, Evelien Geertsema, Nicole Mooren, dank voor jullie grandioze inzet, uitstekende hulp en mooie stages in Amersfoort. Erg leuk om te zien dat jullie inmiddels allemaal een mooie positie hebben en hard aan de weg timmeren. Hiermee bewijzen jullie maar weer de grote waarde van de Technische Geneeskunde!

Arts-onderzoekers Pepijn van Geuns en Rob Tolboom, wat hebben we een fantastische trip naar Miami beleefd! Dank voor die geweldige ' wetenschappelijke' mijlpaal en alle andere events waar jullie bij waren. Ik wens jullie allen succes voor de toekomst!

Lieve familie, vrienden en vriendinnen: veel te veel om allemaal te noemen, maar ontzettend veel dank voor jullie aandacht, support en liefde! 
Geachte weledelgeleerde Heeren van de Ronde Tafel 113 te Utrecht, Wat een prachtavonden heb ik al met jullie beleefd! Tweemaal een Kerstdiner waarbij letterlijk en figuurlijk alle registers open gaan, grandioos! De Dijkavonden, thuistafels, serviceprojecten en weekenden zorgen ervoor dat ik weer eens over wat anders praat dan een robot operatie of een heftige medische casus. Door mijn buitenlandse avonturen en vele verplichtingen in de kliniek, mis ik helaas regelmatig 'de Veurletste', maar jullie blijven prachtige groep gasten! Thanks! Special thanks to George for his marvelous translations and revisions. En Robin: heel veel dank dat ik je laptop mee mocht nemen naar Australië!

The Boys: Brammedaris, Pleasure, Aarsie, Schaapmeister, Slootje, Rode Nelis en sWillem, Wat begon als een geinig vriendengroepje in 2002, Jaar 1 Geneeskunde, is een hechte vriendenclub (met bijbehorende Aquagirls + kids) gebleken waarin lief en leed gedeeld wordt. Als ik aan collega's vertel dat ik op dinsdag naar de Boysnight ga, denken ze dat dit een lugubere en duistere darkroom betreft, waarin skyrimmen de norm is. Wij weten gelukkig wel beter. Eigenlijk zijn jullie vandaag met zijn zevenen mijn paranimfen, die gasten in dat apenpakkie zijn slechts een afgevaardigde delegatie, omdat het anders zo rommelig wordt... Ciao Amici!

Beste Koene, wie kon vermoeden dat jij mij zou komen bijstaan op dit belangrijke moment? Jij bent een trouwe kameraad en een prachtige vent met wie ik altijd kan brullen van het lachen en met wie een oprecht en open gesprek over eender welk onderwerp altijd goed uitpakt. Ik kan nu al uitkijken naar ons volgende tripje! Thanks dat je mij wilt bijstaan vandaag!

Beste Timme, wie kon vermoeden dat jij mij zou komen bijstaan op dit belangrijke moment? Jij bent een trouwe kameraad en een prachtige vent met wie ik altijd kan brullen van het lachen en met wie een oprecht en open gesprek over eender welk onderwerp altijd goed uitpakt. Maar ook niet te vergeten: de volgende van de Boys die promoveert naast een sick drukke baan als Gynaecoloog. Thanks dat je mij wilt bijstaan vandaag!

Lieve Ruud en Lia, Rutger en Mariëlle,

Het is heerlijk om zo te worden opgenomen in een warm Brabants gezin. Nadat jullie mij 'geadopteerd' hebben, hebben we al veel mooie momenten samen beleefd. Er gaan er vast nog veel bij komen! Jullie interesse en steun kan niet genoeg worden benadrukt. Dikke kus, tot snel in Veghel, Grouw, Haarlem of Utrecht!

Joost en Willemijn en Suze en Martijn, Jullie hebben altijd interesse getoond in mijn drukke bestaan. Steeds moest ik weer uitleggen waarom ik nog stééds niet was gepromoveerd. Vandaag kan ik eindelijk die 's' wegpoetsen. Ik ben trots op jullie en kan altijd op jullie rekenen, dank lieve (schoon)broer en (schoon)zus! Wanneer gaan we weer koffie drinken en worstenbroodjes eten? 
Lieve pap en mam,

Toen jullie menneke in 1980 het licht zag, lag de toekomst nog volledig open. Dankzij een warm nest, een liberale opvoeding en een jeugd waarin wij alle ruimte en middelen kregen om ons te ontwikkelen, hebben Suze, Joost en ik al heel wat mooie mijlpalen mogen bereiken. Vandaag wordt er nog een klapper aan toegevoegd! Zonder jullie onvoorwaardelijke liefde, intense steun en vrije geesten, stond ik niet op de plek waar ik nu ben. Jullie zijn geweldige en trotse (groot)ouders en ik hoop nog veel jaren van jullie liefde te kunnen genieten! Houdoe en bedankt voor alles!

Lieve Lenthe,

Nooit eerder was ik zo trots als op 19 augustus 2012. Een perfect kindje! Elke dag met jou en je zusje is een feestje. Heerlijk om te zien hoe jij je hebt ontwikkeld van een piepklein hummeltje naar een hele stoere meid! Ik hou van jou en je grapjes. Jouw knuffels zijn heerlijk! Ik herken mezelf in jouw temperament, geestdrift en energie! Kus van pappa!

Lieve Sterre,

Klein wondertje! Jij maakt ons prachtige gezin compleet! Met je heerlijke brutale snuit en de mooie ogen van je moeder, ben je prachtig! Je zegt altijd de liefste woordjes en hebt altijd de fijnste knuffels! Ik hou van jou en je grapjes. Ik herken mezelf in jouw sociale en zorgzame karakter! Kus van pappa!

Lieve Viev,

Je bent de vrouw van mijn leven! Jij bent degene die mij er dagelijks doorheen sleept. Meestal met een lieve glimlach, soms met een traan, soms hoofdschuddend. "Wat vindt jij nou toch zo leuk aan die ongelofelijke klotebaan van je?" Die vraag heb ik al verschillende malen proberen te beantwoorden. Het chirurgisch vak (ook nog een gecombineerd met een proefschrift schrijven) trekt een zware wissel op een relatie, een gezin en een leven. Daar moet je maar mee om kunnen gaan! Maar jij bent zo heerlijk down to earth en weet alles vlekkeloos te relativeren. Je hebt een drukke baan en managet het hele huishouden ondertussen ook nog eens, bizar! Toen we elkaar (opnieuw!) leerden kennen, stond ik op punt voor een paar maanden te vertrekken naar New York. Je vond het niet tof, maar wilde mijn ambities niet in de weg staan. Dat is altijd zo gebleven! Nadat we die testcase hadden doorstaan, konden we echt de hele wereld aan! Ik heb niets dan bewondering voor de wijze waarop jij een baken van rust bent in tijden van carrière, opleiding, promotie, gezin, buitenlandse stages en andere avonturen! Jij zorgt er regelmatig voor dat deze ongetemde leeuw zich niet vergaloppeert aan een nog geschifter agendavoorstel. Je hebt de mooiste meiden van de wereld onder je vleugels en je geeft mij en de kinderen al je ontelbare liefde. Ik vind je de allerbeste en allerliefste moeder van de wereld en je bent mijn allerliefste en mooiste vrouw! Ik hou van je! 


